\title{
Feasibility of Realizing a Universal Identifier Based on Fiber Bragg Grating Technology
}

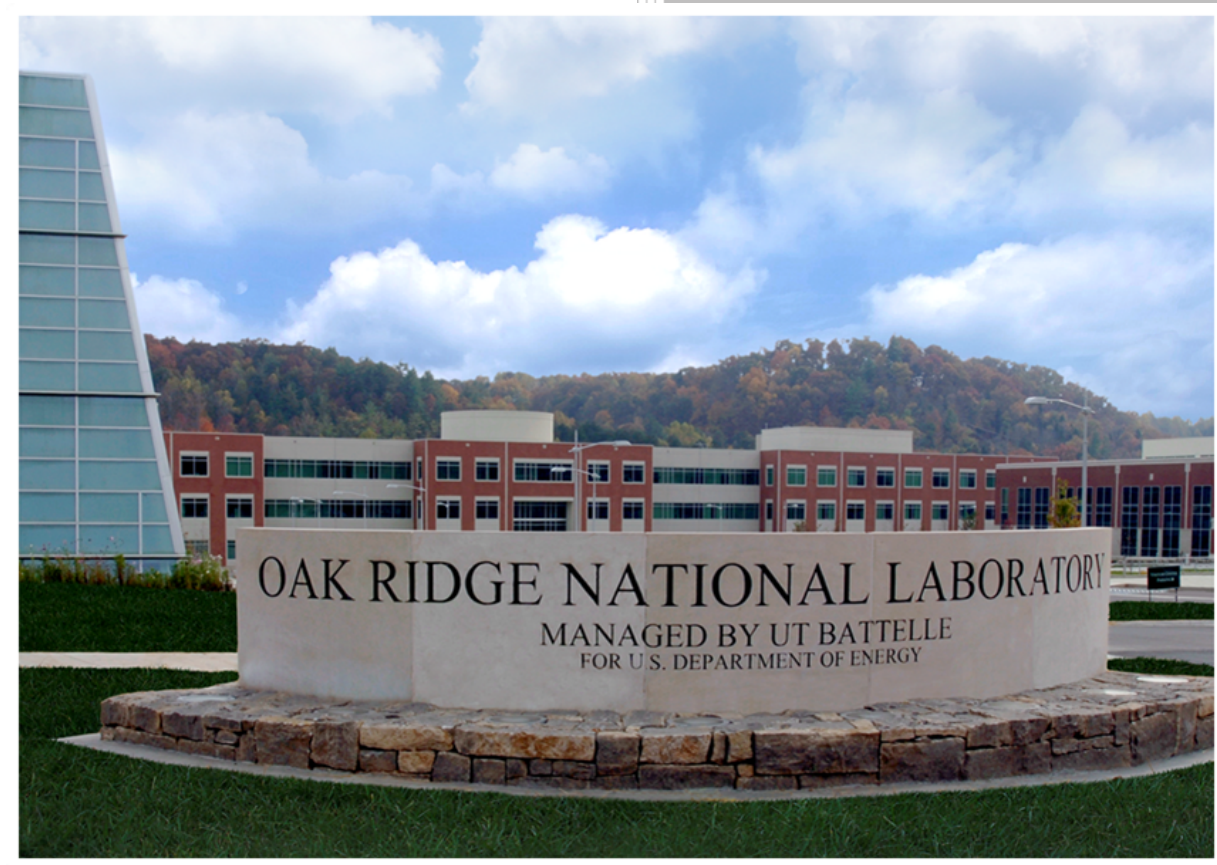

Approved for public release. Distribution is unlimited.

Klaus-Peter Ziock

Phil Evans

Will Ray

James Younkin

February 2020 


\title{
DOCUMENT AVAILABILITY
}

Reports produced after January 1, 1996, are generally available free via US Department of Energy (DOE) SciTech Connect.

Website www.osti.gov

Reports produced before January 1, 1996, may be purchased by members of the public from the following source:

\author{
National Technical Information Service \\ 5285 Port Royal Road \\ Springfield, VA 22161 \\ Telephone 703-605-6000 (1-800-553-6847) \\ TDD 703-487-4639 \\ Fax 703-605-6900 \\ E-mail info@ntis.gov \\ Website http://classic.ntis.gov/
}

Reports are available to DOE employees, DOE contractors, Energy Technology Data Exchange representatives, and International Nuclear Information System representatives from the following source:

Office of Scientific and Technical Information

PO Box 62

Oak Ridge, TN 37831

Telephone 865-576-8401

Fax 865-576-5728

E-mail reports@osti.gov

Website http://www.osti.gov/contact.html

This report was prepared as an account of work sponsored by an agency of the United States Government. Neither the United States Government nor any agency thereof, nor any of their employees, makes any warranty, express or implied, or assumes any legal liability or responsibility for the accuracy, completeness, or usefulness of any information, apparatus, product, or process disclosed, or represents that its use would not infringe privately owned rights. Reference herein to any specific commercial product, process, or service by trade name, trademark, manufacturer, or otherwise, does not necessarily constitute or imply its endorsement, recommendation, or favoring by the United States Government or any agency thereof. The views and opinions of authors expressed herein do not necessarily state or reflect those of the United States Government or any agency thereof. 
Isotope and Fuel Cycle Technology Division

\title{
FEASIBILITY OF REALIZING A UNIVERSAL IDENTIFIER BASED ON FIBER BRAGG GRATING TECHNOLOGY
}

\author{
Klaus-Peter Ziock \\ Phil Evans \\ Will Ray \\ James Younkin
}

February 2020

Prepared by

OAK RIDGE NATIONAL LABORATORY

Oak Ridge, TN 37831-6283

managed by

UT-BATTELLE, LLC

for the

US DEPARTMENT OF ENERGY

under contract DE-AC05-00OR22725 



\section{CONTENTS}

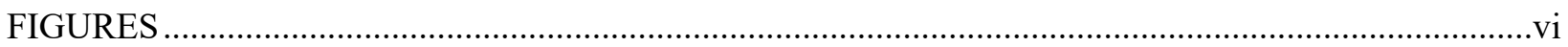

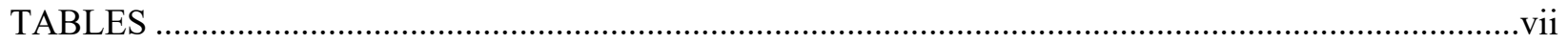

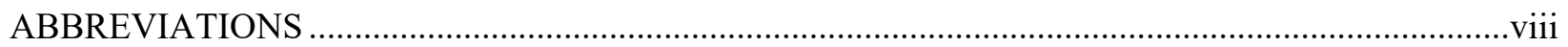

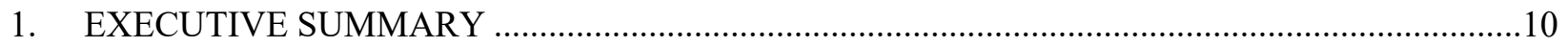

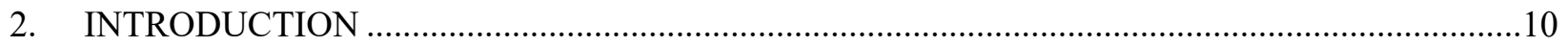

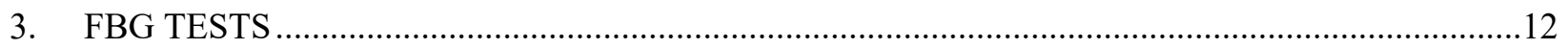

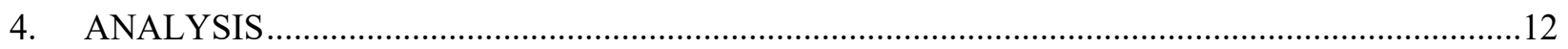

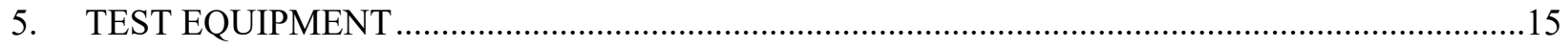

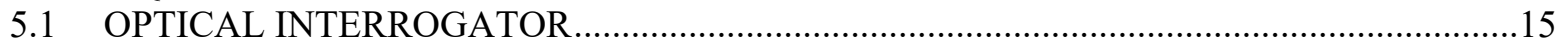

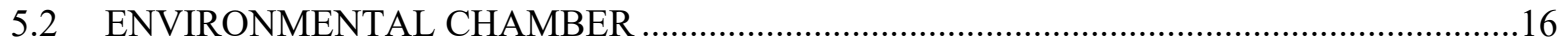

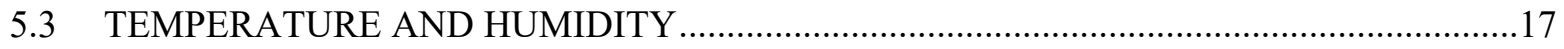

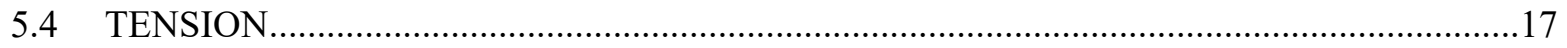

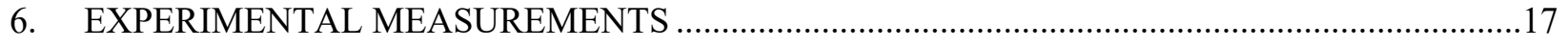

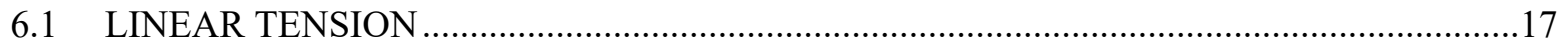

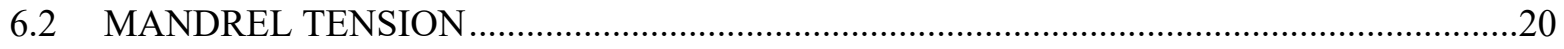

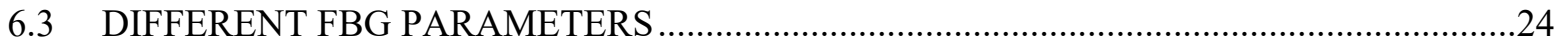

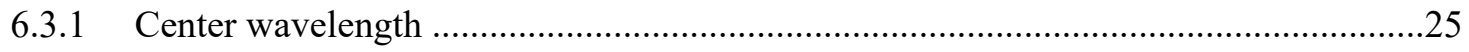

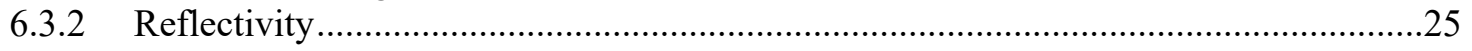

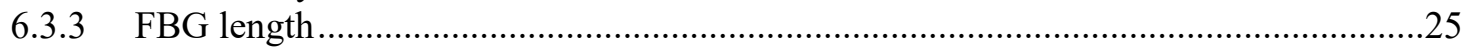

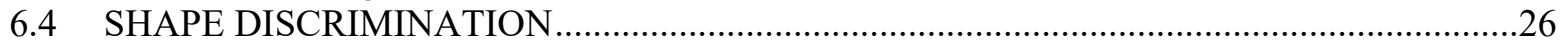

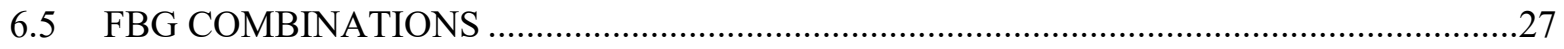

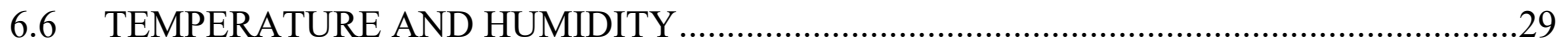

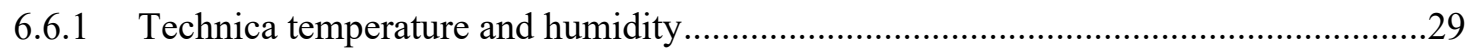

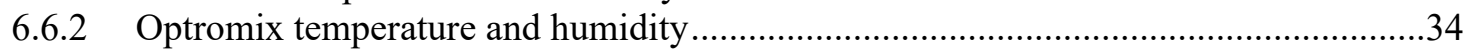

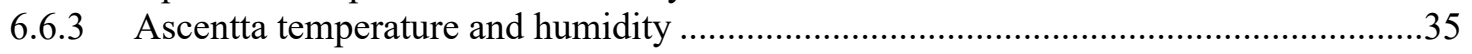

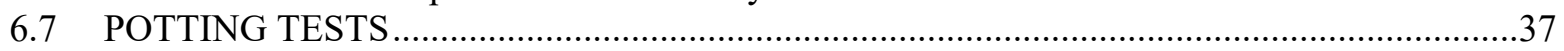

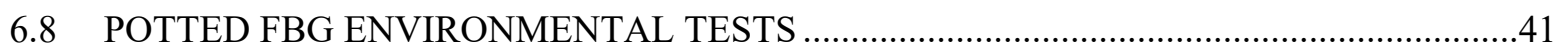

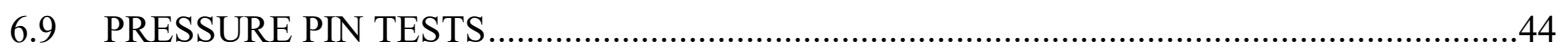

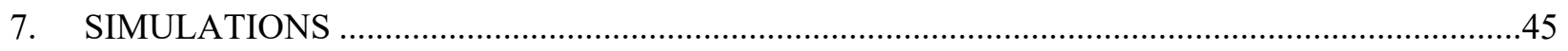

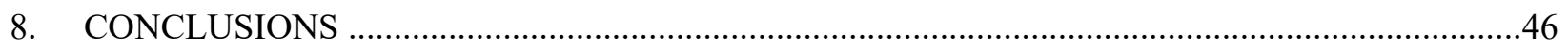

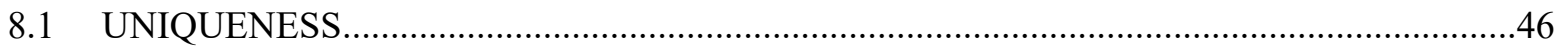

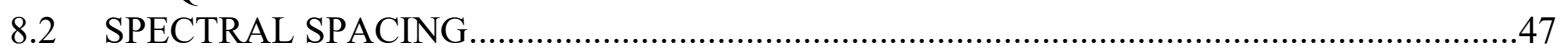

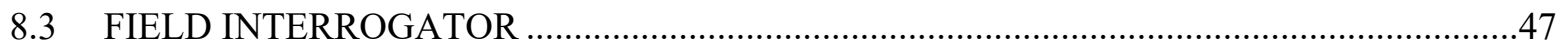

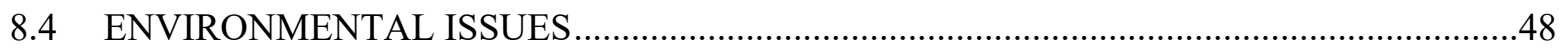

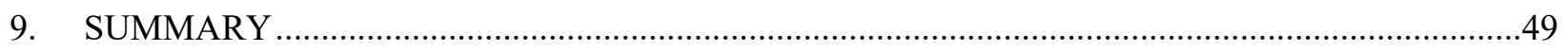

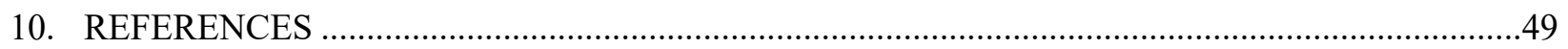




\section{FIGURES}

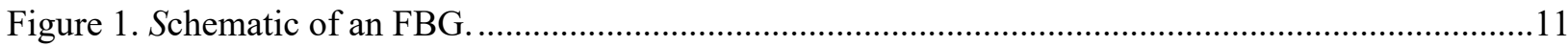

Figure 2. Normalized spectra returned by an apodized and uniform (nonapodized) FBG.......................11

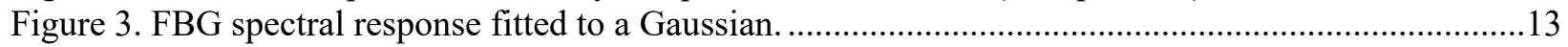

Figure 4. Spectral data from a nonapodized FBG as collected, and plotted as decibels...........................13

Figure 5. Difference correlation results for an FBG plotted linearly and in decibels...............................14

Figure 6. Linear and decibel spectral return and difference correlation for a uniform grating...................15

Figure 7. Optical interrogator obtained for the project........................................................................16

Figure 8. The environmental chamber obtained for the project..............................................................17

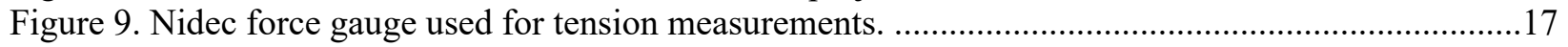

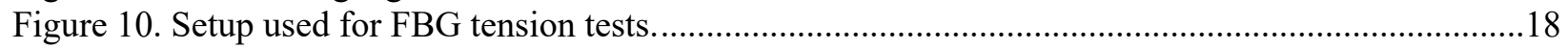

Figure 11. Sample linear tension results obtained for an acrylate-coated FBG.....................................18

Figure 12. Spectral shift from uniform tension applied to an acrylate-coated FBG. ...............................19

Figure 13. Histogram of the slopes of wavelength vs tension for the Technica FBGs.............................19

Figure 14. Results for an acrylate-coated FBG with a $45^{\circ}$ wrap around a 0.5 in. diameter mandrel. .........20

Figure 15. FBG results with different linear polarizations for even and odd spectra ...............................20

Figure 16. Force versus lambda, and the difference-correlation value for different polarizaitons..............21

Figure 17. Optromix acrylate FBG change in spectral return different tensions......................................23

Figure 18. Results for Ascentta acrylate-coated FBG wrapped on a 0.5 in. mandrel with the

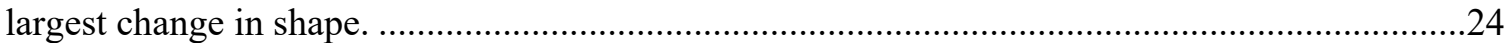

Figure 19. Change in shape for the Ascentta FBG with a large shape change wrapped $90^{\circ}$ on a

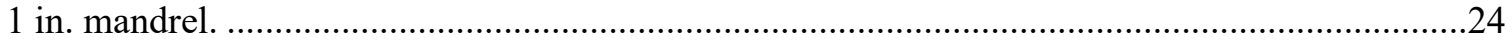

Figure 20. Response for gratings with differing reflectivities. .........................................................25

Figure 21. Plot of the bandwidth vs the inverse of the FBG length....................................................26

Figure 22. Difference-correlation values returned for FBGs with themselves and with others. .................27

Figure 23. Individual response from separate $2 \mathrm{~mm}$ long FBGs and a $4 \mathrm{~mm}$ long FBG..........................28

Figure 24. The results obtained from the model (2) compared to the data collected from the two

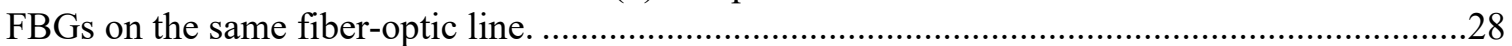

Figure 25. Change in peak wavelength as a function of temperature for four different FBGs.....................30

Figure 26. Variation in chamber relative humidity as a function of temperature for the thermal

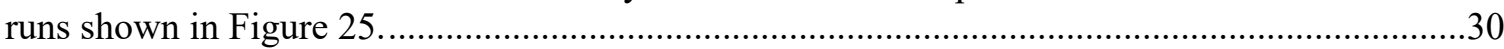

Figure 27. Changes in peak wavelength as a function of relative humidity for the four FBGs in

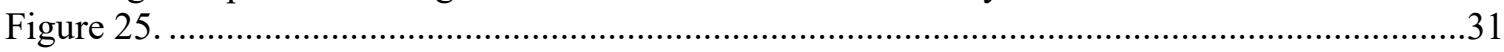

Figure 28. Thermal run results for acrylate and polyimide coated Ascentta FBGs.................................32

Figure 29. Humidity run results for acrylate and polyimed-coated Technica FBGs. .................................33

Figure 30. FBG spectral response for an Ascentta device at two different relative humidities...................33

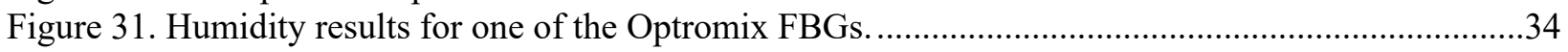

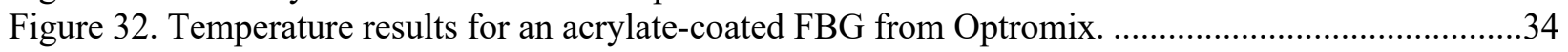

Figure 33. Humidity response of a partial Optromix polyimide-coated FBG. ….....................................35

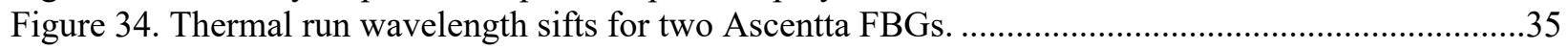

Figure 35. Humidity run wavelength shifts for two Ascentta FBGs. .......................................................

Figure 36. Change in the Ascentta polyimide coated-FBG spectral return. .............................................36

Figure 37. Humidity run wavelength shifts for a bare Ascentta FBG. .................................................37

Figure 38. Spectra returned from an unbroken FBG and the from two partial FBG segemnts..................37

Figure 39. Results obtained on potting a Technica polyimide-coated FBG in volumetrically stable

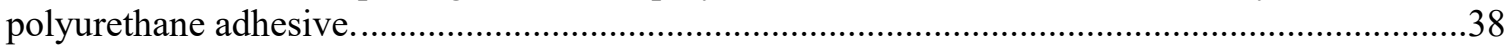

Figure 40. Results obtained on potting a Technica polyimide-coated FBG in expanding polyurethane adhesive. 
Figure 41. Results obtained with Technica acrylate-coated FBG potted in expanding polyurethane adhesive.

Figure 42. Results obtained with Technica acrylate-coated FBG potted into a form with rubber fingers using an expanding polyurethane adhesive.

Figure 43. Results obtained with two halves of a bare FBG potted in expanding polyurethane and 5-minute epoxy.

Figure 44. Changes in the environmental conditions during the test results of Figure 43........................40

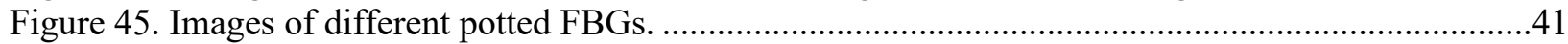

Figure 46. Environmental test results of bare, potted FBG segments....................................................42

Figure 47. Thermal and humidity results for epoxy-potted and polyurethane-potted acrylatecoated Technica FBGs.

Figure 48. Temperature and humidity results for a polyimide-coated FBG potted in polyurethane...........44

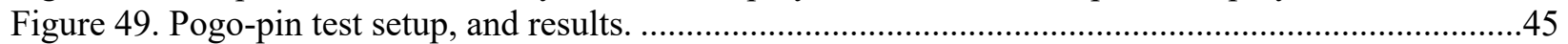

Figure 50. Simulated and measured signal from an FBG with a center wavelength of 1,558 nm. ...........46

Figure 51. Wavelength and linewidth measurements for the PurePhotonics PPCL550 compact tunable laser diode.

\section{TABLES}

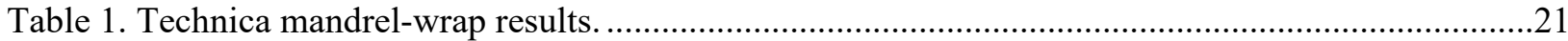

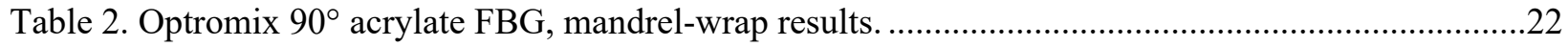

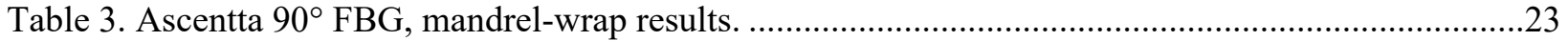

Table 4. Wavelength shifts of stressed FBGs bent around a 0.5 in. mandrel. .........................................46 


\section{ABBREVIATIONS}

CTE coefficient of thermal expansion

FBG fiber Bragg grating

IR infrared

UID universal identifier

USB universal serial bus 



\section{FEASIBILITY OF REALIZING A UNIVERSAL IDENTIFIER BASED ON FIBER BRAGG GRATING TECHNOLOGY}

\section{EXECUTIVE SUMMARY}

Over the past year we have collected data on different fiber Bragg gratings (FBG) with the end goal of understanding their suitability for use in a new type of unique identifier (UID) that could be used in both tags and seals for high-value, high-risk assets. The ideal UID will have a complex signature that allows items to be uniquely identified by that signature. Tamper indication must be an integral part of the tag/seal process with measurable changes to the device signature indicative of any attempt to tamper with the monitored item. Further, the signature must be so difficult to reproduce that even state-actors with significant resources at their disposal will be discouraged from attempting it. Finally, readout verification of the product must be simple and secure, while providing red-light/green-light results in real time.

To evaluate how well an FBG-based UID could meet these goals, we collected data on a large number of FBG samples from different vendors. Measurements included the response of the devices to uniform tension, tension when wrapped on a mandrel, and the response to changes in temperature and humidity, as well as the effect of simple potting in expanding and nonexpanding potting materials. We tested FBG samples written into single-mode fibers that were coated with the two commonly used materials, polyimide and acrylate and gratings that were both uniform and those with an apodized response (i.e., the intensity of the grating was varied to minimize sidelobes).

The result of the work to date did not find any significant issues with the design concept for a UID based on this technology. Specific findings include a baseline design comprising five FBGs with distinct spectral returns analyzed using a difference-correlation approach. To realize this device, careful attention must be given to the impact of environmental factors on the UID response, limiting the properties of the potting compounds and FBG coating materials that can be used.

\section{INTRODUCTION}

Fiber Bragg gratings (FBG) are optical devices written into fiber optics using intense UV light to create small, permanent, spatial changes in the index of refraction of the fiber core. Typically, the changes are written with a regular periodicity commensurate with the wavelength of the light sent down the fiber (Figure 1). The resulting optical structure acts as a Bragg reflector for incident light in resonance with the structure, returning the light back through the fiber where it can be detected. A typical response from a uniform FBG (Figure 2) has sidelobes that add some spectral complexity to the reflected spectrum. These can be suppressed by varying the intensity of the pattern in the fiber core at the beginning and end of the FBG. Such "apodized" gratings (Figure 2) provide a cleaner return with a central reflection wavelength that can be monitored autonomously to look for changes in the wavelength.

Small changes in the periodicity of the FBG will shift the central frequency, and this forms the basis for a commercial industry that relies on the devices. An FBG's periodicity will vary because of changes in external parameters such as temperature, tension, and pressure. Hence the FBGs can be used as sensors to monitor for changes in these external parameters. In fact, by building the FBGs into simple mechanical structures, sensitive sensors can be designed that specifically monitor for specific external parameters. For instance, they are widely used in civil engineering to monitor structural stresses. FBGs have several advantages over other mechanical systems including longevity and robustness. They also do not require an electrical connection (and therefore are free from electrical interference), and they may be used for long-distance remote monitoring because of the ability to transmit light long distances through fiber optics. Finally, spectral shifts are much easier to track than absolute amplitude measurements. 

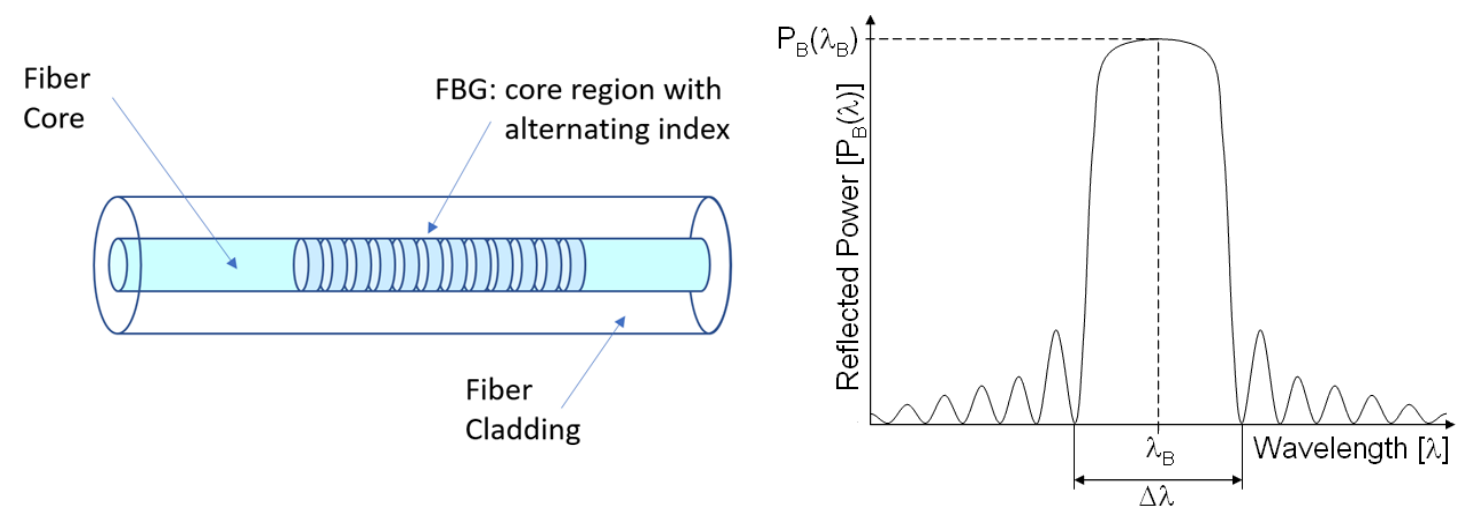

Figure 1. Left, schematic of an FBG with the index of refraction of the core of a fiber optic strand varied as a function of location. Right, theoretical reflection spectrum from a nonapodized FBG.
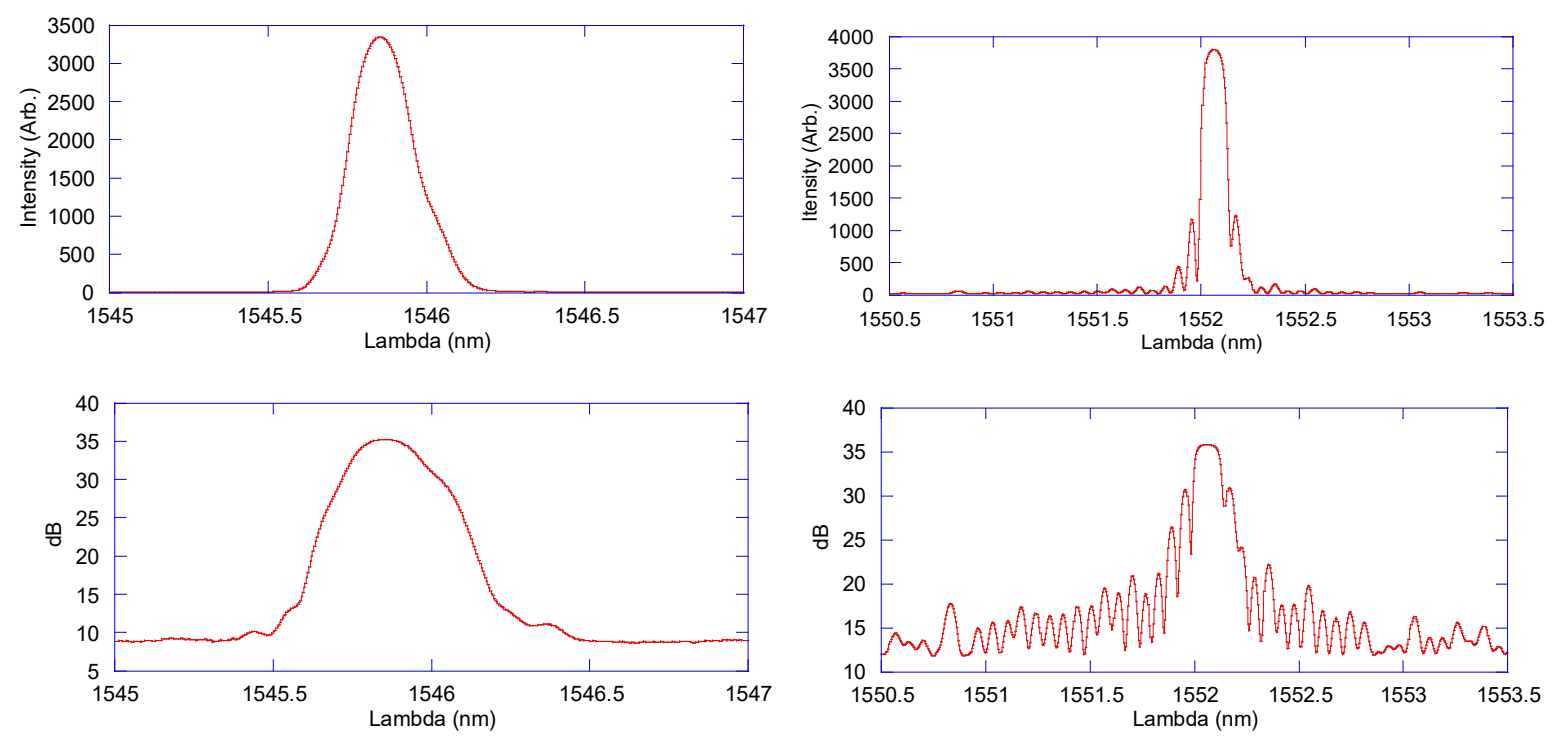

Figure 2. Normalized spectra returned by an apodized (left) and uniform (nonapodized) FBG (right). The upper row shows the linear response, whereas the lower row is given in decibels (logarithmic scaling), accentuating lower level structure.

Many of the advantages for commercial applications (FBGs have been used for numerous applications, including strain/temperature measurements in a variety of applications [1]) represent desirable attributes for applications safeguarding nuclear materials. For instance, the lack of an electrical connection can be important for applications where explosives might be encountered, and the inherent spectral readout implies tolerance for high radiation fields. The existence of the commercial industry is also advantageous to this project, providing both low-cost gratings and sophisticated readout devices to study their application in an UID.

The UID concept central to this project includes the use of several gratings, each with its own spectral response, serially located on a single fiber-optic cable. The FBGs will be potted into the UID with a randomized stress field that alters each FBG's response to make a complex spectral signature that will be virtually impossible to counterfeit. This report chronicles the first steps toward that goal, reporting on an initial study into FBGs to understand if this overall concept is valid. To that end, a high-resolution FBG readout device (optical interrogator) was obtained and simple measurements were taken from a selection of FBGs. 


\section{FBG TESTS}

To determine their suitability for use in an UID, a series of tests were conducted that included the response of different FBGs to external stimuli, including linear tension and tension applied to FBGs wrapped on a mandrel, as well as changes due to temperature and humidity. The tests were conducted on a selection of single FBGs acquired from four vendors. All were manufactured in single-mode fibers with an overall diameter of $\sim 250 \mu \mathrm{m}$, half of which is the glass fiber and half is a protective coating. There are two standard coating types available, acrylate and polyimide, and both types were tested. FBGs from one of the manufacturers had an additional outer coating that rendered them useless for further work because it varied the stress transmitted to the fiber in an uncontrolled fashion.

To write the FBG into a fiber, the manufacturer must first remove the protective coating surrounding the glass fiber. After the FBG is written, that coating is reapplied manually, which results in a less uniform result than on the rest of the fiber. In fact, the coating was sufficiently perturbed on some of the fibers such that the coating interfered with the mandrel tests. Various vendors produced better results with different coating types, and this will factor into vendor selection based on the coating type selected.

FBGs with a range of physical properties were obtained from one of the manufacturers. Variations included the center wavelength, the length of the FBG (from $2 \mathrm{~mm}$ to $1 \mathrm{~cm}$ ), different reflectivities (from $\sim 45 \%$ to $\sim 95 \%)$, and FBG bandwidths $(0.25 \mathrm{~nm}$ and $0.5 \mathrm{~nm})$. We also obtained copies of several of the FBGs that were written to have the same design specifications. Comparison of the response of "duplicate" FBGs provides a measure of how well a given FBG design can be reproduced.

Initial potting tests were performed to see if random stresses in the FBG could be induced to intentionally alter their spectral signatures. The potted FBGs were also subjected to thermal and humidity variations to see how the composite structure behaved. One of the biggest concerns to the UID development is that changes to external parameters will affect an UID's spectral signature and cause device identification to become problematic.

Finally, a simple proof-of-principle experiment was conducted on the concept of creating individual user keys. The idea behind such keys is that by pressing on the UID at different locations and with different forces (such as might be generated by an array of pogo pins with different spring constants), the UID signature would be altered to create a unique, key-dependent response. This is an additional confounding factor making defeating the devices particularly difficult.

\section{ANALYSIS}

To understand the effect of external parameters on the FBG response, some form of metric is required. Ideally, this will include wavelength shifts as well as spectral shape. Initial analysis was performed by fitting a Gaussian to the spectral return (Figure 3). This was considered inadequate both because the Gaussian was a poor match to the shape of the apodized FBGs initially measured, and because it addresses none of the spectral complexity that might be encountered in a nonapodized FBG or a multiFBG device. To include some measure of the latter, a difference-correlation analysis was developed that compared the shape returned by an FBG in its unstressed state with the shape returned when under stress. For the comparison, both the unstressed and stressed spectra are first self-normalized by the maximum peak value (maximum minus the minimum). The spectral region of the unstressed shape is selected manually to cover the full spectral reflection, typically several times the nominal width of the reflection (e.g., an FBG with a nominal $0.5 \mathrm{~nm}$ bandwidth would include a total width of $1-2 \mathrm{~nm}$ ). The value returned by the difference correlation of a particular wavelength value is then the sum of the absolute 
values of the differences between the normalized shape, $S_{i}$, and normalized spectral data, $D_{j}$, offset by the wavelength of the comparison location:

$$
V_{c c}(\lambda)=\sum_{i=0}^{n}\left|S_{i}-D_{\lambda-i}\right|
$$

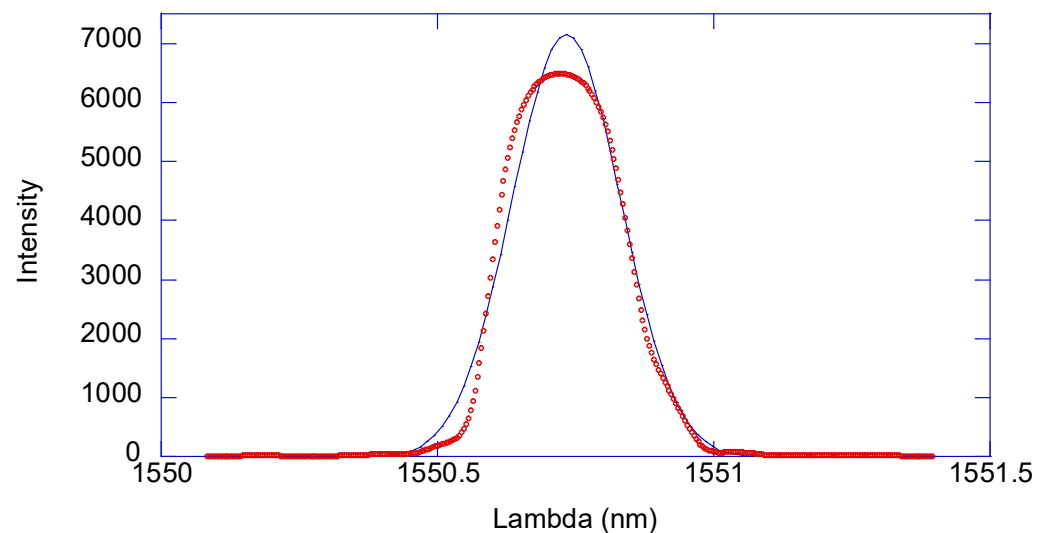

Figure 3. FBG spectral response fitted to a Gaussian (blue line). Although it is clearly not the correct shape, the fit provided an initial metric to monitor change in wavelength vs applied stress.

When applied to the spectrum from which the shape was taken, this will return zero when the value is calculated with the shape at its original location in the spectrum (Figure 4). However, because the data are frequently rebinned to spectra with lower resolution and because the difference-correlation is performed at the full spectral resolution $(1 \mathrm{pm})$, the actual value returned by the analysis code will be greater than zero. In addition, data were generally collected as $10 \mathrm{~s}$ integrations, comprising $\sim 40$ spectra. The shape used for comparison was generally taken as an average of the summed shapes collected in the unstressed condition, and this shape was then compared to each of the individual shapes collected with the subsequent stressor conditions. The reported results are the average of the $\sim 40$ individual values returned by the analysis using Eq. (1) and the variance of those 40 values used to determine the precision of the results, (e.g., the error bars on the plots).
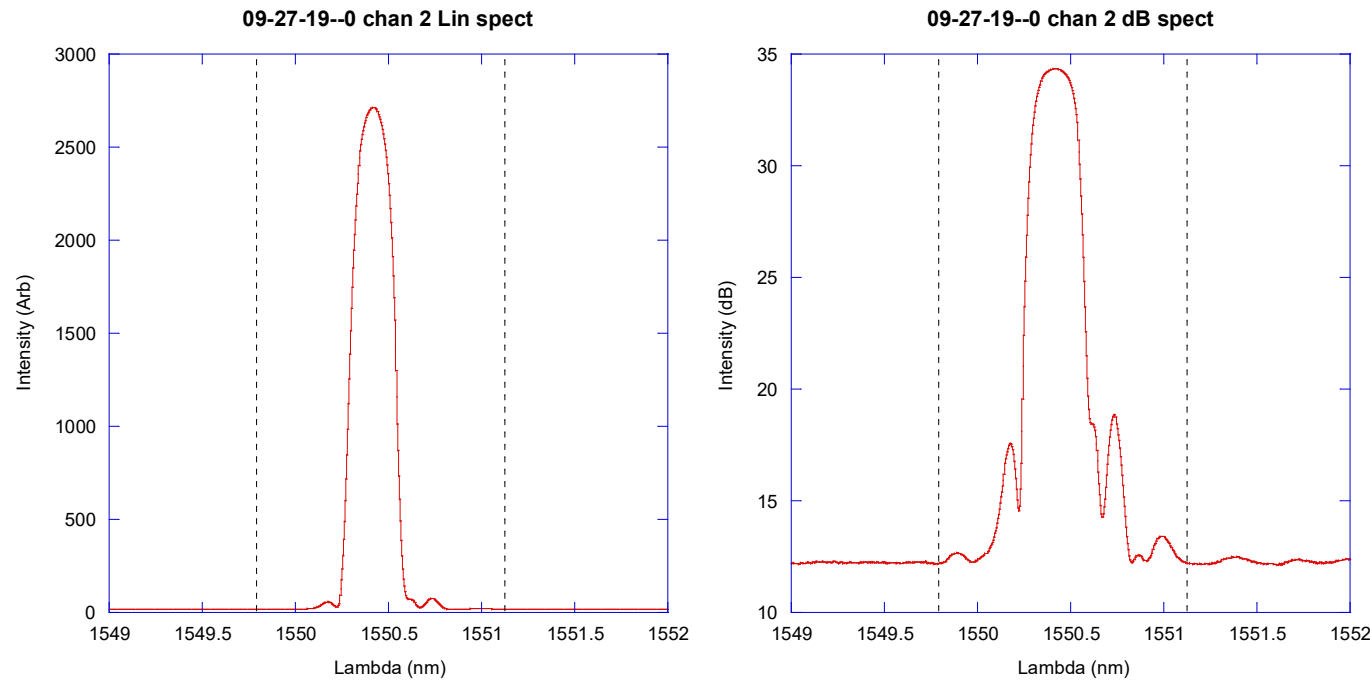

Figure 4. Spectral data from a nonapodized FBG as collected (left) and plotted as decibels (right). The dashed lines indicate the region of the spectrum selected for the shape used in performing the difference-correlation analysis. 
In practice, one could interpolate using several channels around the minimum returned $V_{c c}$ to find a refined position for the peak location. However, given the spectral resolution of the interrogator, simple use of the spectral channel with the minimum value was deemed sufficiently accurate for this work. Hence, the match location is reported based simply on the wavelength bin that returned the minimum $V_{c c}$ and that value was reported as the $V_{c c}$ value. $^{1}$

Note that the use of difference correlation reduces the value of a single FBG to three numbers: the wavelength of the minimum location, the value of the difference correlation at the minimum, and the selfnormalization value applied to the analyzed spectrum. Of these, the minimum value is most sensitive to the shape of the response. Even a more complex multi-FBG spectrum will return only three values, and whether or not this is sufficiently accurate for analysis of a full UID will require further study. At a minimum, this analysis technique provides an excellent starting point for a more complex analysis that could include applying Eq. (1) to subdivided spectral regions or by finding and tracking significant peaks within the overall UID band pass.

Finally, we note that many of the difference-correlation results were obtained using the spectral intensity expressed in decibels, rather than the linear response of the interrogator. This use accentuates the contribution from the lower intensity sidelobes of the returned spectra (Figures 5 and 6).
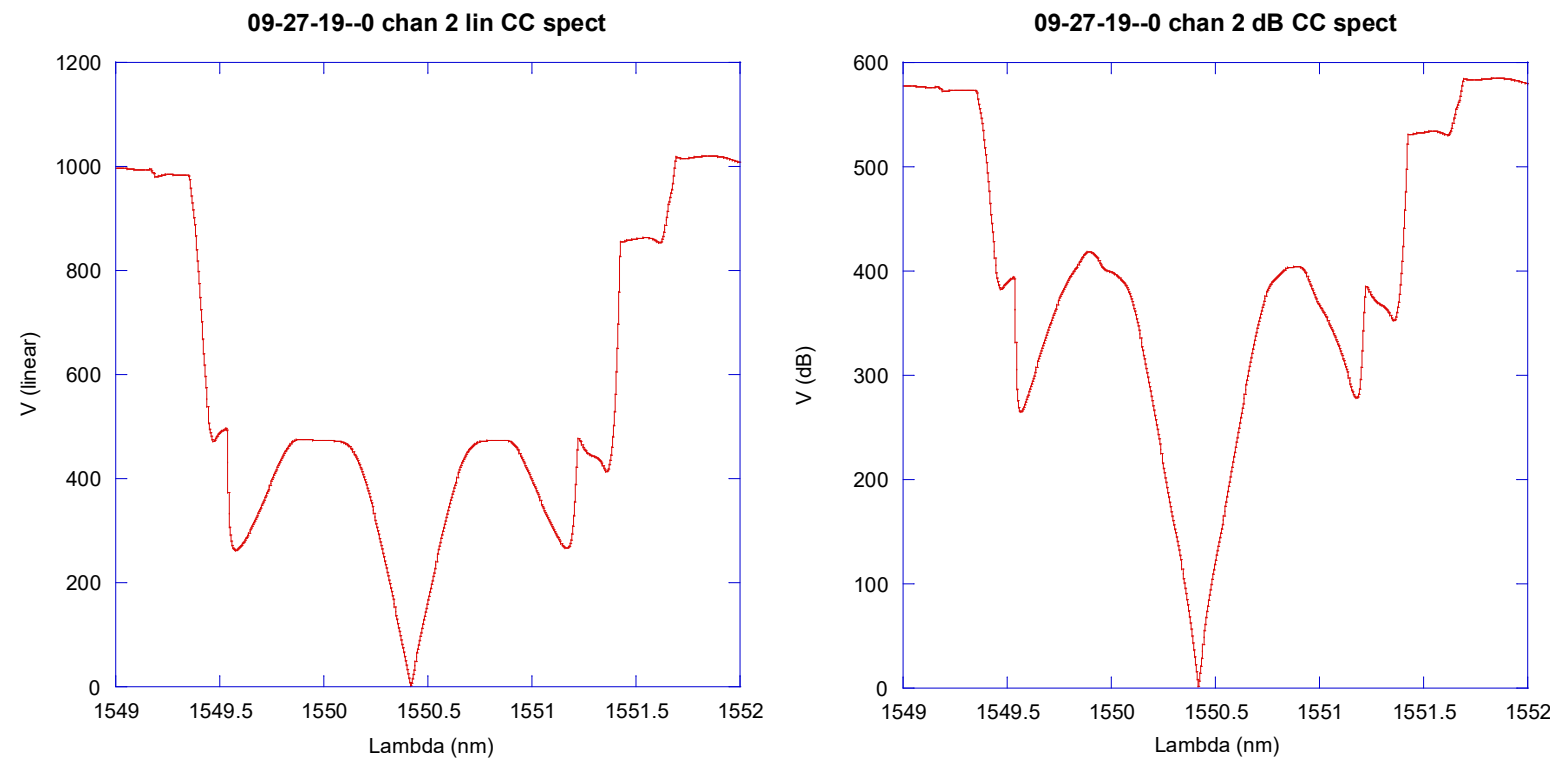

Figure 5. Difference-correlation results for the spectra with spectral shape regions shown in Figure 4 for linear (left) and decibel data (right).

\footnotetext{
${ }^{1}$ In the plots, this parameter is interchangeably labeled $V$ or min.
} 

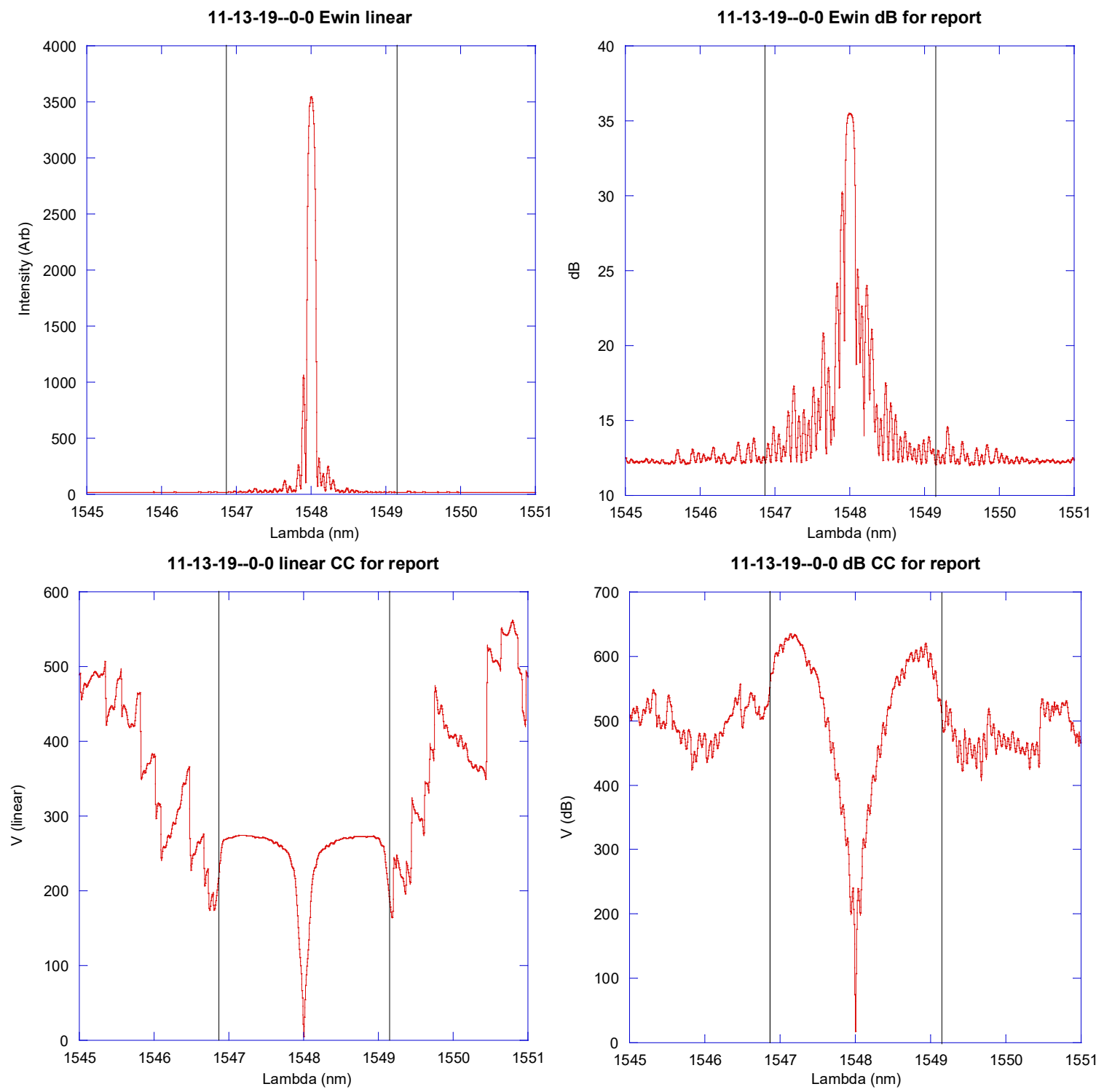

Figure 6. Linear (left) and decibel spectral return (top row) and difference correlation (bottom row) for a uniform grating.

\section{TEST EQUIPMENT}

\subsection{OPTICAL INTERROGATOR}

To achieve widespread application, any UID design will require a low-cost, hand-held readout device that can be easily used in the field. Ideally, such an optical interrogator would have a very high spectral resolution to enable using the full complexity of an UID spectral signature. In practice, increased spectral resolution comes with an increase in price so that units suitable for field use will require a trade-off from what is possible in a laboratory setting. To understand such trade-offs, an optical interrogator was selected for these measurements that significantly exceeds the performance of likely fieldable units. To that end, a search for suitable laboratory-grade instruments was conducted, culminating in the procurement of a FAZT-I4G optical interrogator (Figure 7) manufactured by Faz Technology [2]. 


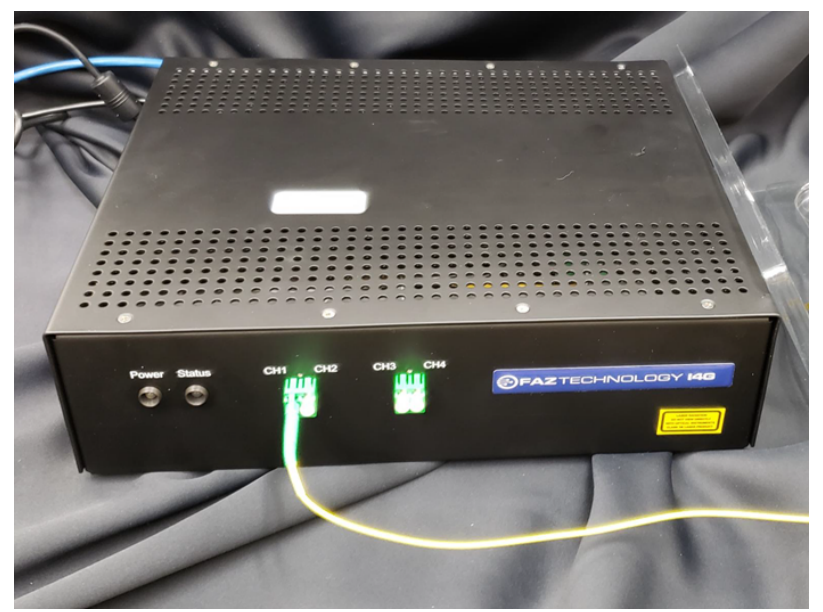

Figure 7. Optical interrogator obtained for the project can read FBGs on four ports with a spectral output rate of $4 \mathrm{~Hz}$.

The FAZT-I4G offers turn-key simplicity, excellent signal-to-noise ratio ( $\sim 40 \mathrm{~dB})$, and exceptional spectral resolution (30 MHz linewidth) over the infrared (IR) C-band (1529.0-1568.2 nm). It is also better suited to experimental use than many competitors, in that the full spectrum (with 1 pm steps) can be easily read out and saved on a host computer at $4 \mathrm{~Hz}$, including the data from any or all of the device's four fiber-optic ports. It will also alternate linear polarization on sequential spectra, allowing a study of this parameter as well. Many of the competing devices only report the central wavelength returned by an FBG, a feature desirable for tracking the response of sensors to external stimuli, but not relevant to this work. Although FBGs can be manufactured across a wide spectral range, the desire to limit our work to commercially available products led to selection of the IR band. Commercial availability is influenced by the telecommunications industry, which routinely uses fibers with attenuations of much less than a decibel per kilometer [3]. A primary selection factor was the spectral resolution, which was several times that of the nearest competitor. It did require that the work be conducted in IR C band, which is toward the long wavelength end of the IR bands used by the telecommunications industry. The one potential downside of using longer wavelengths is a greater loss of light for a given fiber bend radius [4], which might require a somewhat larger UID device.

\subsection{ENVIRONMENTAL CHAMBER}

One of the primary concerns about the overall UID design is the impact of environmental conditions on the signature of an UID. Comprised of FBGs in a randomized stressor field, the effect of varying environmental conditions on that stressor field will change the spectral response, and this could so alter the UID signature that the ability to identify it or determine whether it has been altered could be compromised. To study temperature and humidity, a thermal/humidity chamber from Associated Environment Systems (Figure 8) was acquired [5]. The chamber is capable of providing temperature stability of $\pm 0.5^{\circ} \mathrm{C}$ for a range of $-20^{\circ} \mathrm{C}$ to $94^{\circ} \mathrm{C}$ and a relative humidity stability of $\pm 2 \%$ over the range $10 \%-98 \%$. However, the humidity can only be controlled for specific temperature ranges (Figure 8). Consequently, tests of thermal response were run over a (typical) range of $-15^{\circ} \mathrm{C}$ to $80^{\circ} \mathrm{C}$ without humidity control, and the humidity was run from $20 \%$ to $95 \%$ at a temperature of $45^{\circ} \mathrm{C}$. 

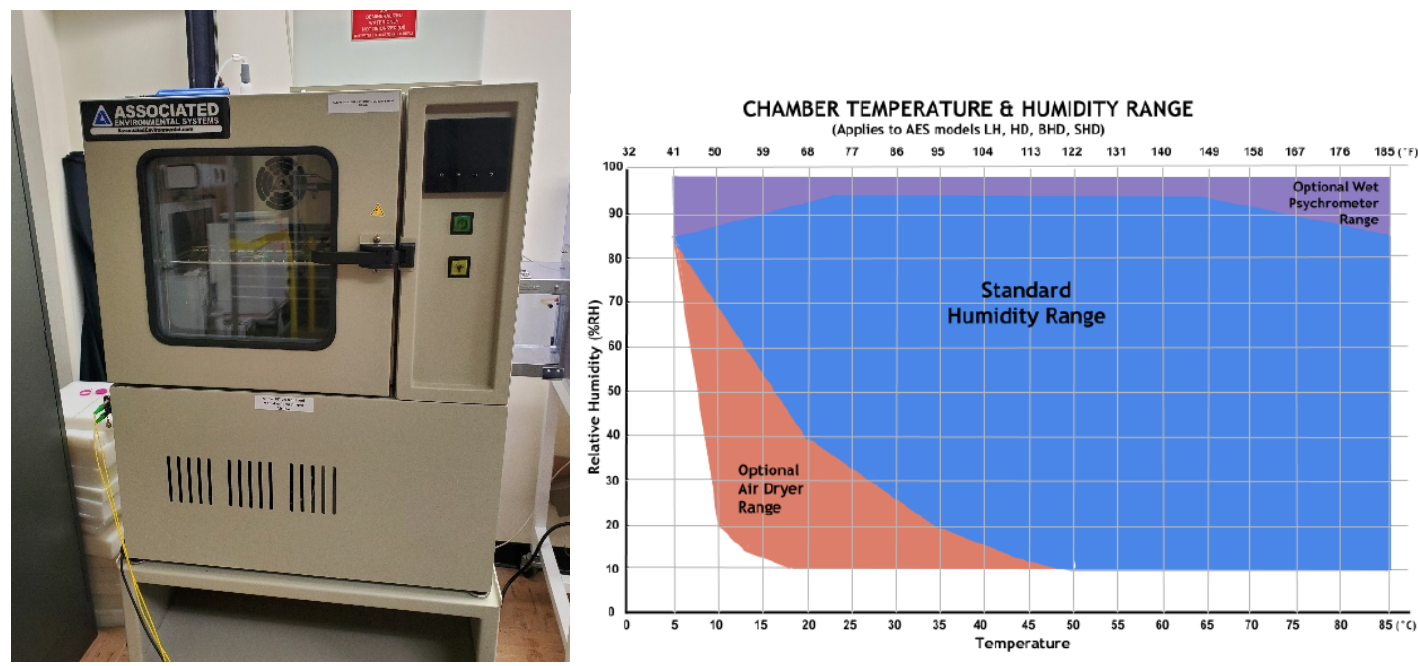

Figure 8. The environmental chamber used for these measurements (left). (Right) Accessible humidity values as a function of temperature with different chamber options. The chamber used in the tests could control the humidity in the standard humidity range (blue) [5].

\subsection{TEMPERATURE AND HUMIDITY}

To record temperature and humidity during the various tests, a low-cost, USB-based Phidget dual sensor [6] was used. (For the chamber-based environmental tests, the values reported by the chamber were used.)

\subsection{TENSION}

FBGs respond to mechanical stress, and one source of stress is tension on the fiber and FBG. Simple linear tension was applied to the FBG by stretching it between a linear spring scale mounted on a computer-controlled linear stage. As the stage position was varied, the tension was read out using a Nidec force gauge (Figure 9) [7].

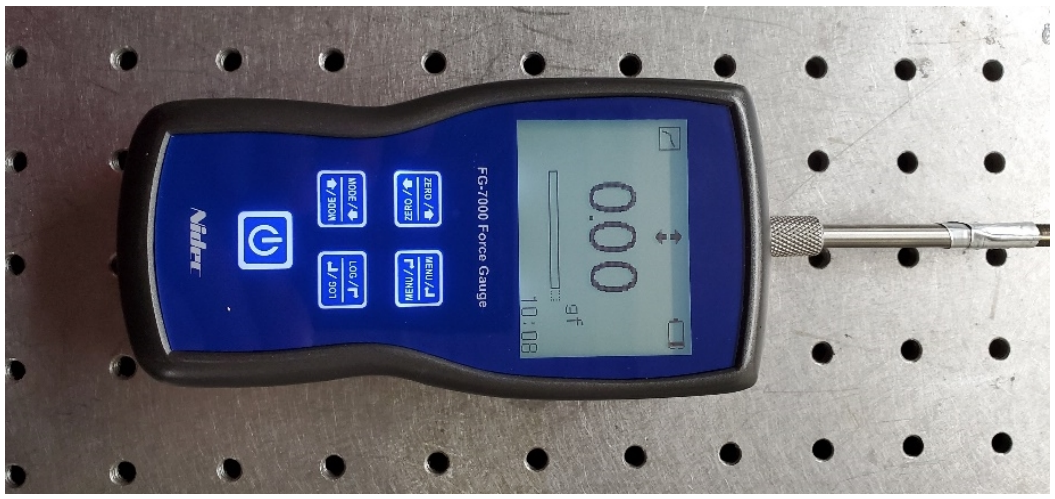

Figure 9. Nidec force gauge used for tension measurements.

\section{EXPERIMENTAL MEASUREMENTS}

\subsection{LINEAR TENSION}

Perhaps the simplest stress to apply (and interpret) to the FBGs is linear tension. This should linearly change the wavelength of the reflected spectrum, with minimal change to the shape. To test the response, 
several FBGs were stretched by attaching one end of the fiber containing the FBG to a spring scale mounted to a linear translation stage, and the other end was attached to a force meter (Figure 10). Data were collected under computer control by stepping the translation stage in steps of equal distance so that the force went from a minimum of a few grams to a maximum of $\sim 50 \mathrm{~g}$ (earlier tests only went to $\sim 20 \mathrm{~g}$ ). Figure 11 shows a typical result, where all three values returned by the difference-correlation analysis are plotted as a function of tension. As stated above, each point represents the average of $10 \mathrm{~s}$ of data ( 40 spectra) with the error bars obtained from the distribution of the different spectra at a given tension. As can be seen, the peak wavelength increases linearly, but the difference-correlation minimum and the normalization values change very little. This is an indication that the shape of the spectrum changes very little as seen in Figure 12, where representative spectra are plotted.

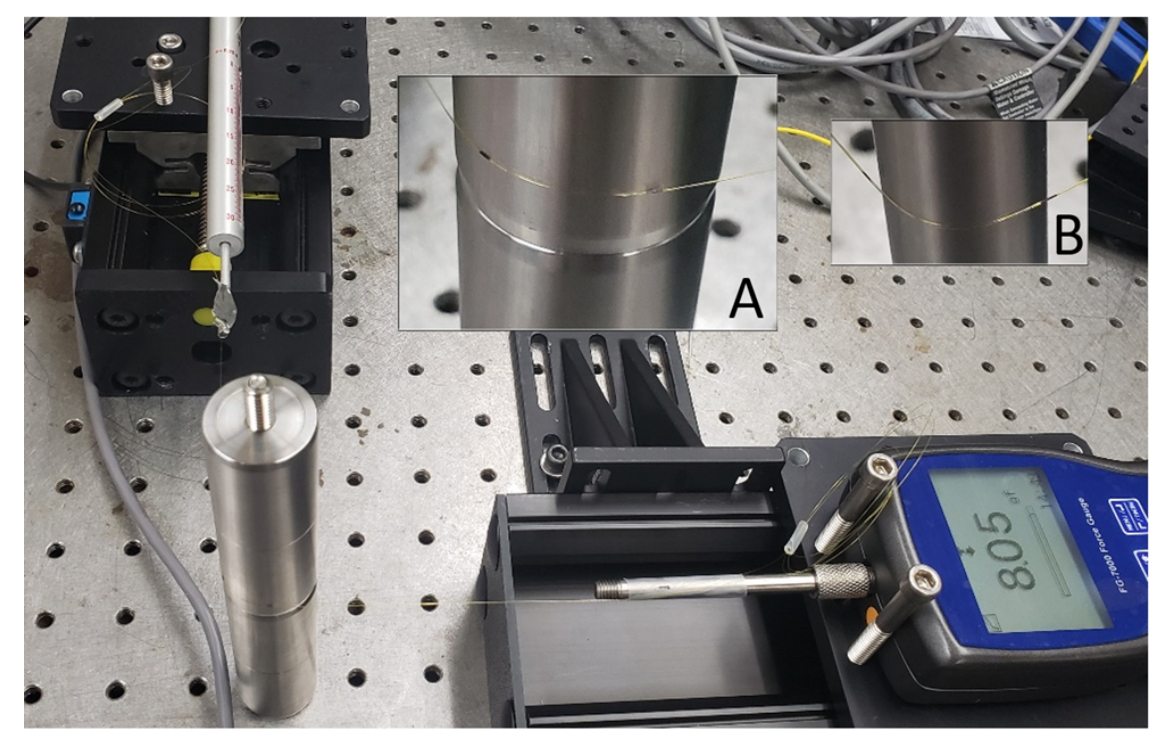

Figure 10. FBG tension tests. A spring scale attached to a computer controlled linear translation stage (upper left) is used to vary the tension of the fiber, measured by a linear force gauge (lower right). In this case the FBG undergoes a $90^{\circ}$ wrap on a 1 in. diameter mandrel. A close-up of the contact region for the 1 in. (A) and 0.5 in.

(B) mandrels are shown in the insets. The FBG is between the two black marks visible on the fibers.
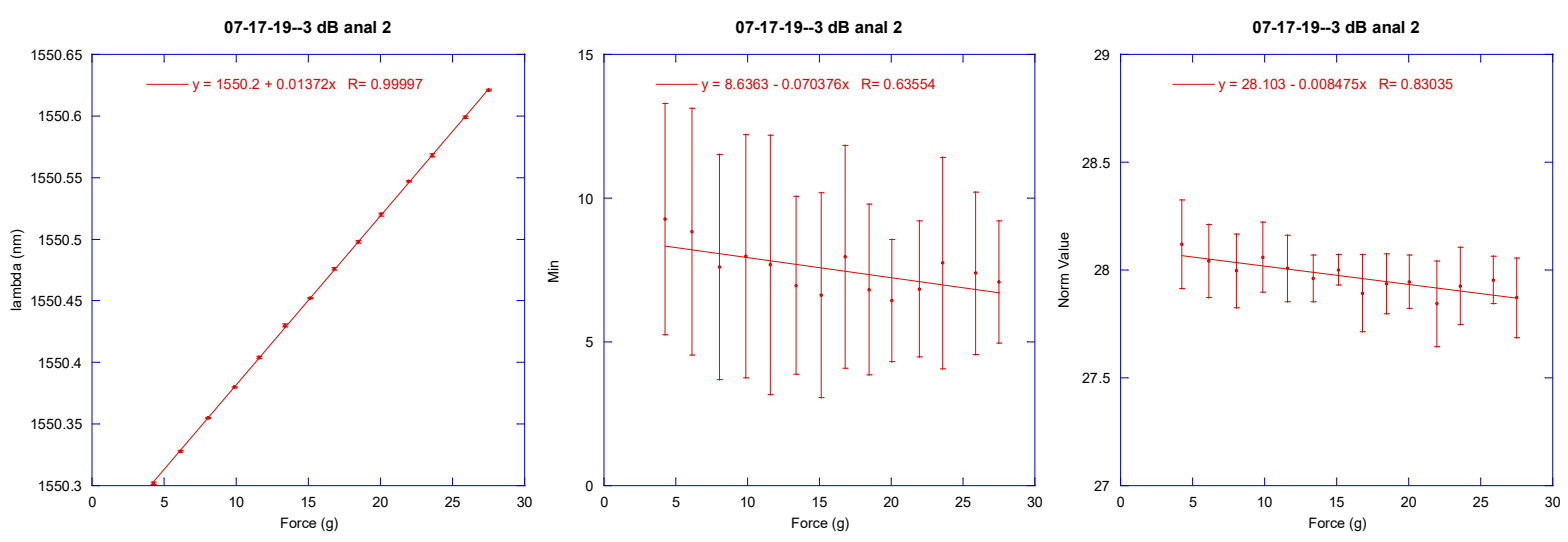

Figure 11. Sample linear tension results obtained for an acrylate-coated fiber. 

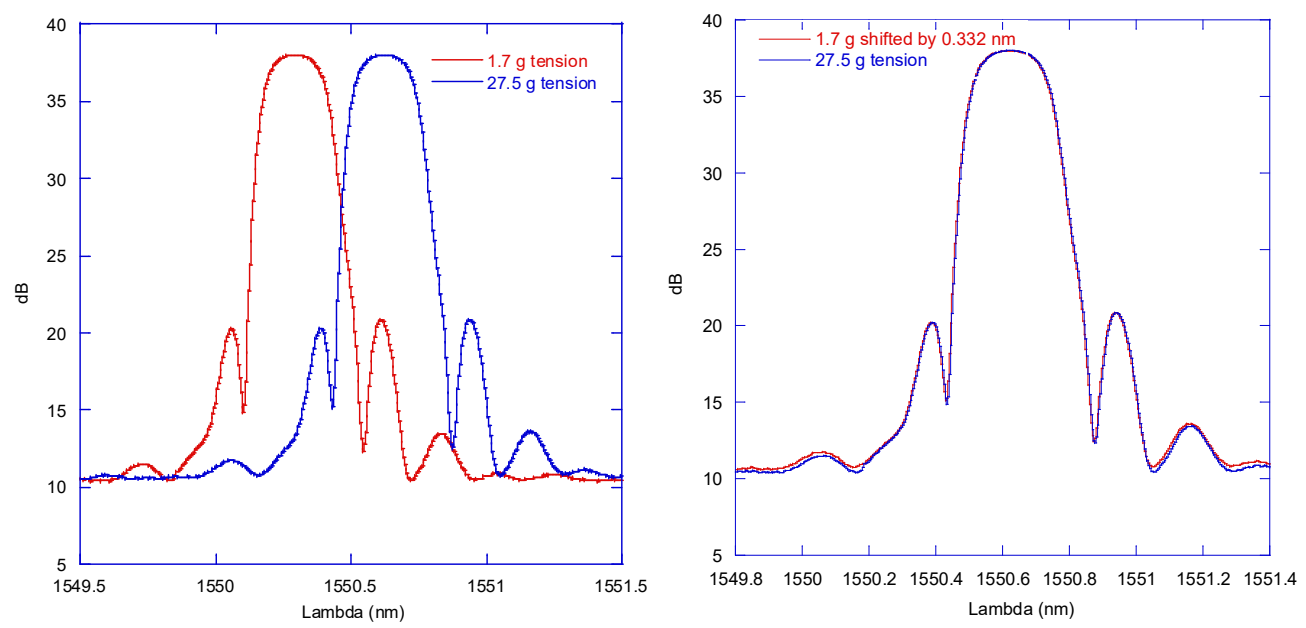

Figure 12. Left, shift in the reflected spectrum due to $27.5 \mathrm{~g}$ uniform tension applied to an Technica Acrylatecoated FBG. Right, the same data with the $1.7 \mathrm{~g}$ waveform shifted by $0.332 \mathrm{~nm}$, showing that there is very little change to the shape of the response.

The overall results for the Technica FBGs are presented in Figure 13, which plots a histogram of slopes of the wavelength shift versus tension. There is a small difference between the two coating types, which is not surprising because the materials will have different properties under tension. There were only three samples of each of the coating types for the Ascentta fibers (acrylate, polyimide, and bare) and only three samples of acrylate and polyimide coatings from Optromix, so a histogram was not considered useful. Instead, a scatterplot with the different types labeled was created (Figure 13, right). It shows a smaller difference between the polyimide and acrylate-coated FBGs from Optromix and no significant correlation for those from Ascentta. However, we note that the Ascentta polyimide coating was poor at best, showing stretches of FBG with little or no coating with some blobs on one side of the FBG (not surrounding the fiber) at other locations. Consequently, it is unlikely to significantly alter the stress carried by the fiber. Overall, the spectral shapes did not change significantly for any of the fibers during these tests, indicating that to change the shape of the FBGs in an UID will require something other than simple linear tension.
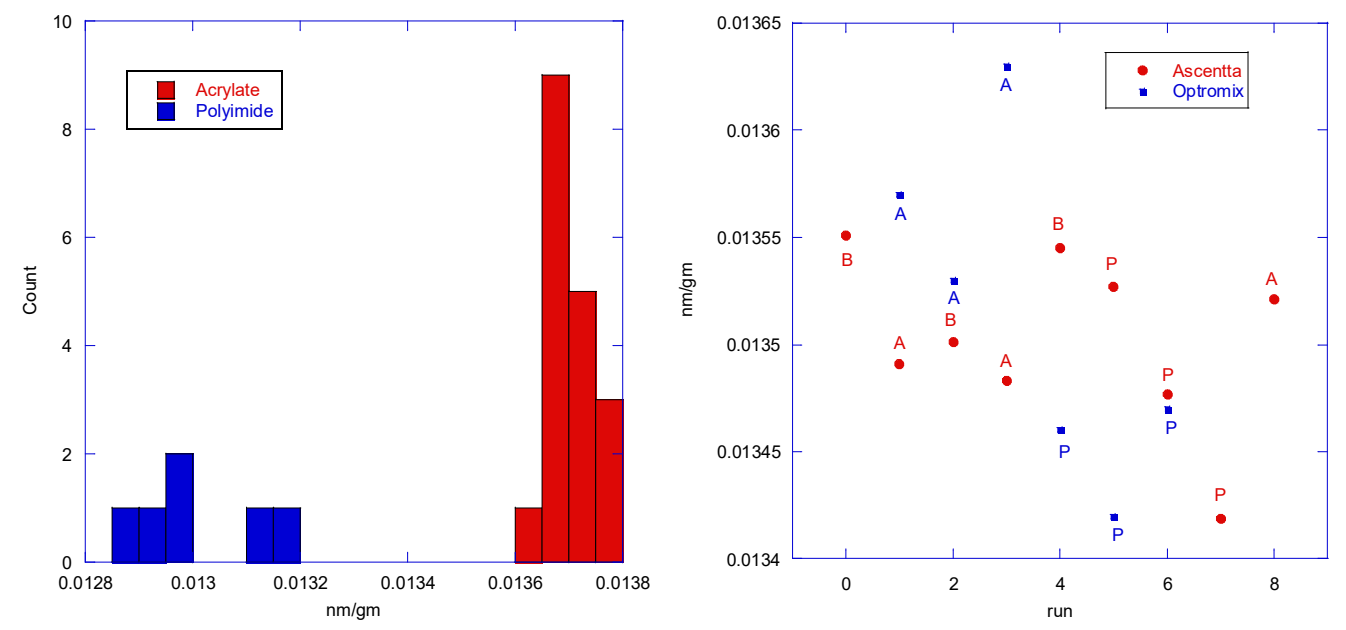

Figure 13. Left, histogram of the slope for the fits to the FBG wavelength vs tension for the Technica FBGs. There is a clear separation between the polyimide- and acrylate-coated fibers. Right, results for the Optromix FBGs show a smaller separation, and the Ascentta results are mixed between the bare (B), acrylate-coated (A), and polyimide-coated (P) FBGs. 


\subsection{MANDREL TENSION}

As shown in Figure 10, tension measurements were also performed by wrapping the fiber around one of two mandrels. Diameters of 1 in. and 0.5 in. were used with the FBG in contact with, and centered on, the contact area. For most of the tests, a $90^{\circ}$ bend was used with the stage and spring scale on one side of the mandrel and the force gauge on the other. For early tests with the polyimide-coated FBGs from Technica, tests were also run at $45^{\circ}$ and $135^{\circ}$. Several FBGs failed at the $135^{\circ}$ angle on the smaller mandrel, and those tests were abandoned. In general, at $45^{\circ}$, the results from both posts were similar, with a linear shift in center wavelength and small shifts in the minimum of $\mathrm{V}_{\mathrm{cc}}$. Many of those data were collected taking advantage of the interrogator's ability to alternate the linear polarization $\left(90^{\circ}\right)$ of the light on every other spectrum. In general, there were small changes in the response between the polarization states

(Figures 14-16). One polarization generally had a larger returned peak value than the other. Because the polarization state was not returned with the spectrum, which spectrum (odd or even in number during an acquisition) clearly changed from run to run. In principle, a cut on one property (e.g., the normalization value) allows the two polarizations to be disentangled and should even allow for the use of results from only one polarization. However, it was not clear that the results between the two states were sufficiently different to justify the added complication of using polarized light, and this approach was not pursued further.
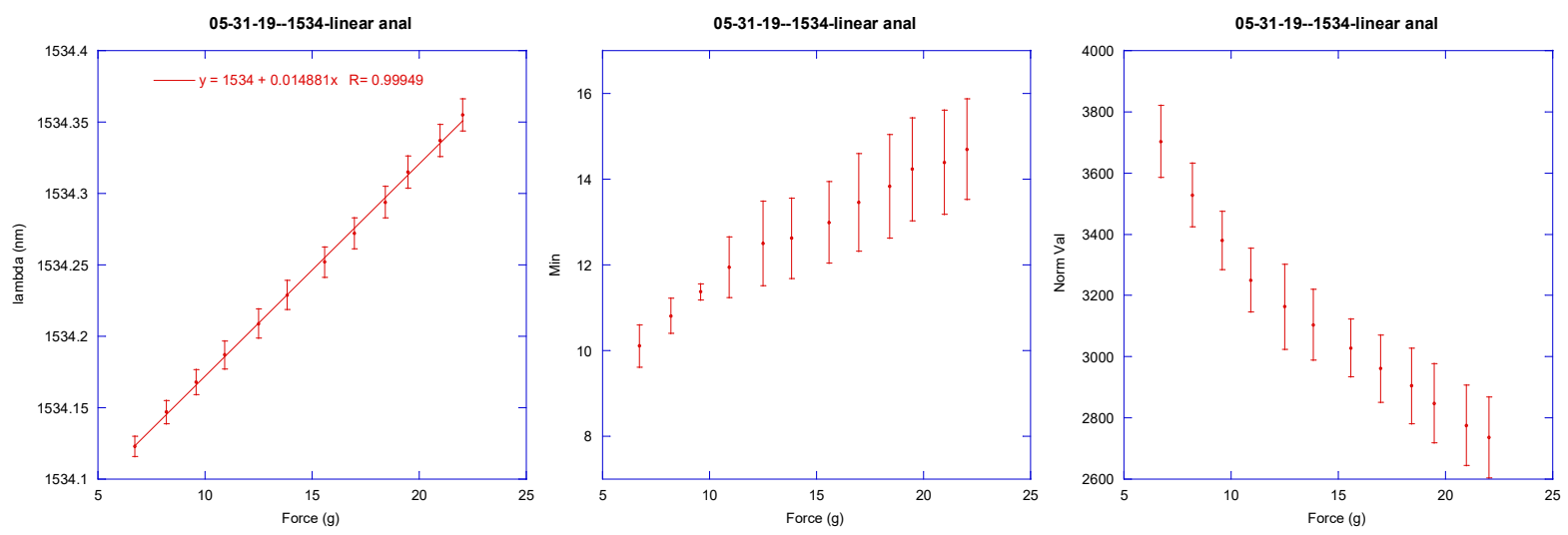

Figure 14. Results for an acrylate-coated FBG with a $45^{\circ}$ wrap around a 0.5 in. diameter mandrel. The large error bars are due the use of alternating polarizations.
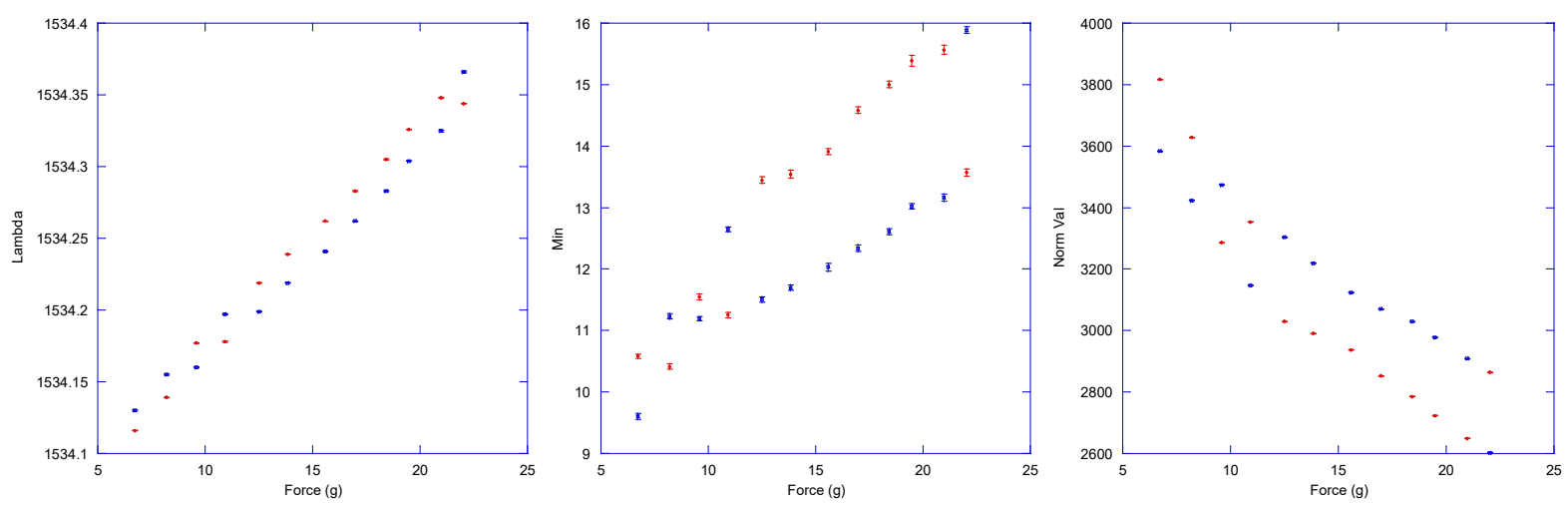

Figure 15. The same data as in Figure 14 but with the even and odd spectra of each acquisition analyzed separately. Because the polarization state of the system is not included in the output data, which polarization corresponds to "odd-numbered" and which is "even-numbered" clearly alternates between data points. This could be disentangled by selecting data from only one of the noncrossing traces, as in the upper and lower norm value curves in the plot on the right (see Figure 16). 

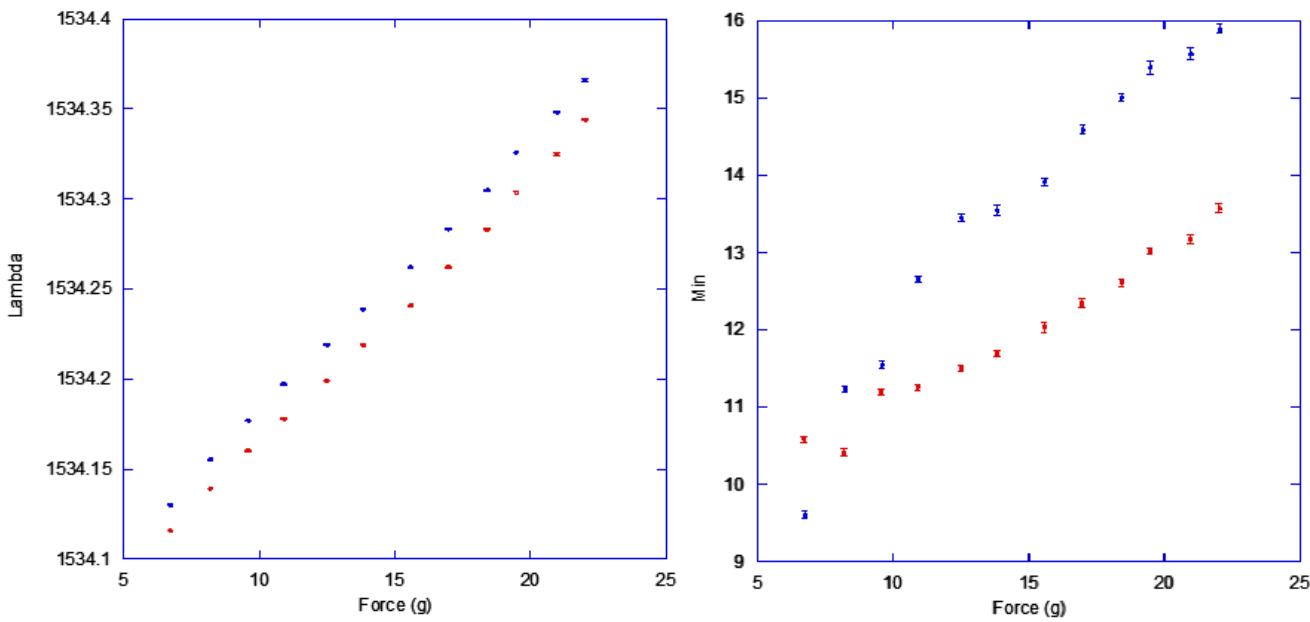

Figure 16. Force versus lambda (left), and force versus the difference-correlation value selecting on the upper (red) and lower (blue) curves of the plot of normalization value on the right of Figure 15.

The results obtained on the mandrels are of particular interest because the planned UID design has the FBG threaded through an array of pins before potting to alter the spectral signatures. To first order, results obtained across all three wrap angles $\left(45^{\circ}, 90^{\circ}\right.$, and $\left.135^{\circ}\right)$ on both the $0.5 \mathrm{in}$. and $1 \mathrm{in}$. mandrels indicate the change in wavelength with tension is linear. The change in shape and the norm values obtained for the Technica polyimide fibers are summarized in Table 1 . The values in the table are averages of the differences between the minima and maxima obtained from the plots of the $\mathrm{V}$ and normalization as a function of tension for each of five FBGs at each angle. Clearly, wrapping the FBG around the larger mandrel has little effect on its spectral shape (V value). This is not the case for the smaller mandrel. Somewhat surprisingly, the largest change in the shape occurs for the smallest wrap angle. However, on reflection this can be traced to the fact that the FBG is in contact with the mandrel for only part of its length, so there are unique responses from the two regions (the one in contact with the mandrel and the rest of the FBG). For the larger wrap angles, the full length of the FBG is in contact with the mandrel, so there is only one type of response. The other significant effect is that there is a clear loss in signal intensity, as evidenced by the large change in normalization value. This is expected from the tight bend radius, which is known to cause transmission losses in fibers optics [4] and has been observed in these fibers when loops are pulled too tight. Of course, the third observation is that several of the FBGs broke after the largest wrap angle tests, indicating that some care is required for "tight" turns.

Table 1. Technica mandrel-wrap results.

\begin{tabular}{|l|l|l|l|l|l|l|}
\hline \multicolumn{4}{|l|}{ V Value } & \multicolumn{3}{l|}{ Norm Val } \\
\hline Wrap angle & $\mathbf{4 5}^{\circ}$ & $\mathbf{9 0}^{\circ}$ & $\mathbf{1 3 5}^{\circ}$ & $\mathbf{4 5}^{\circ}$ & $\mathbf{9 0}^{\circ}$ & $\mathbf{1 4 5}^{\circ}$ \\
\hline 0.5 in. mandrel & 34.1 & 18.8 & 12.1 & 0.5 & 1.6 & 2.8 \\
\hline 1.0 in. mandrel & 8.6 & 10.7 & 12.6 & 0.2 & 0.3 & 0.2 \\
\hline
\end{tabular}

Mandrel tests were not performed on the Technica acrylate-coated FBGs because the surface of the recoated FBGs were rough and tacky. This created too much stiction so that the tension applied on one side of the mandrel was not uniformly transferred to the force meter on its far side. Attempts to use a water-based lubricant or an interstitial bearing material such as Teflon tape or graphite-coated paper were unsuccessful. 
The Optromix fiber mandrel tests were only conducted with a wrap angle of $90^{\circ}$. The biggest difference between these and the Technica fibers was the ability to test the two coating types, as their acrylate recoated FBGs did not have the same stiction issues as those from Technica. However, there were issues with the polyimide fibers breaking. In the end, all three polyimide-coated samples broke, two in the FBG and the other one broke twice, once on either side of the FBG near the connectors. (Although the sample size is small, it hints that polyimide from Optromix may be a poor choice for durability.) Thus, only two polyimide results were obtained, one on each mandrel diameter, but with different FBGs, making drawing meaningful conclusions difficult. For the 0.5 in. mandrel, the minimum $\mathrm{V}$ value changed by 13 while for the one-inch mandrel the result was smaller at 11. This is similar to the trend for the Technica results at $90^{\circ}$, but the half-inch-mandrel change is significantly smaller than for those devices. That said, there were some changes in procedure, and with a single measurement from different samples it is not clear that the Optromix results are significant. Turning to the acrylate-coated FBGs, results were obtained for all three samples and are summarized in Table 2.

Table 2. Optromix $90^{\circ}$ acrylate FBG, mandrel-wrap results.

\begin{tabular}{|l|l|l|}
\hline & Min Value & Norm Value \\
\hline 0.5" mandrel & 21.4 & 0.1 \\
\hline $\mathbf{1 . 0}$ " mandrel & 23.6 & 0.2 \\
\hline
\end{tabular}

Similar to the Optromix polyimide, and unlike the Technica polyimide FBGs, the results for the two mandrel sizes are very similar, although the magnitude for both is similar to the larger change of the Technica half-inch results. The biggest surprise is that the magnitude of the shape differences for the 1inch mandrel are comparable to those for the half-inch mandrel. One difference between these FBGs and those from Technica are very high reflectivities, as indicated by the squarer shape of the reflection profile. Sample shapes for the half-inch mandrel results with the biggest change in the Min value are given in Figure 17.

As with the Optromix FBGs, the Ascentta fiber mandrel tests were only conducted with a wrap angle of $90^{\circ}$. The biggest differences between these and the devices from other manufacturers was their nonapodized spectral response, and the ability to test the different coating types, including uncoated FBGs. Unfortunately, the polyimide coatings provided by Ascentta were visually inferior to their acrylate coatings. Two samples of each of the fiber types were tested. The average results of each type are given in Table 3 .

With only two FBGs of each type tested, inferences from the results must be drawn with caution. This is especially true for the polyimide results because the coatings viewed under a microscope were clearly inferior to those from other manufacturers. The bare fiber results indicate that the smaller diameter mandrel imposes greater stress on the FBG, resulting in larger changes in the shape. The acrylate shape changes are the largest of those observed, possibly indicating greater adhesion between the recoatings and the fibers and that the adhesions may be nonuniform. One of the more interesting results with the acrylate coatings is that for one of the two, the 1 in. mandrel results were significantly larger (69.3) than for the other (23.3), whereas for the 0.5 in. mandrel the values were much closer (52.9 and 66.6, respectively). This is likely an indication of nonuniformity in the coating-fiber interaction, likely exacerbated by how the FBG contacted the mandrel. There was very little signal loss in these fibers for either bend radius. Sample plots of the response for the acrylate fiber that had the biggest shape changes are shown in Figures 18 and 19. 

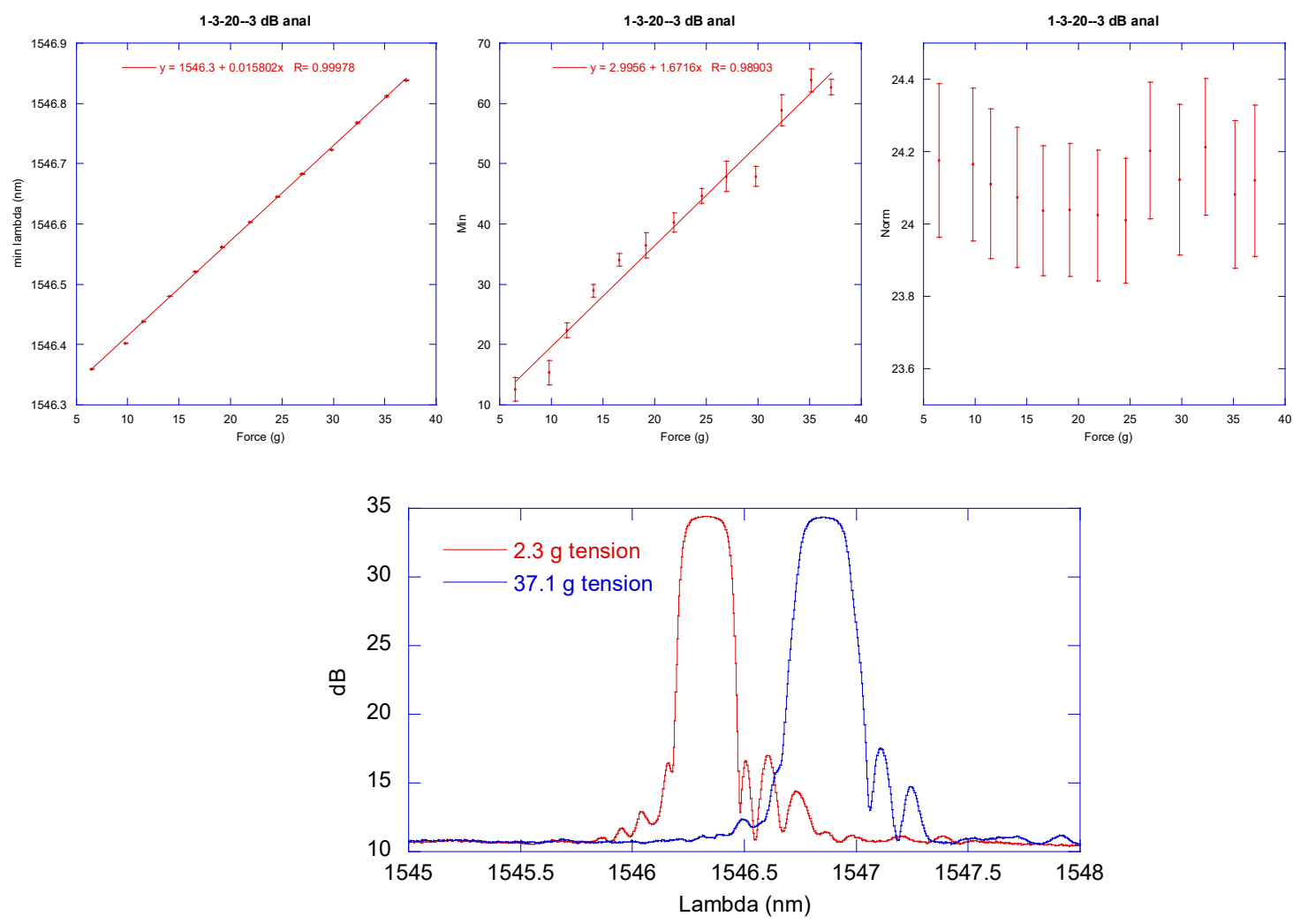

Figure 17. Optromix acrylate FBG change in spectral return for $2.3 \mathrm{~g}$ tension (red) vs $\mathbf{3 7 . 1} \mathrm{g}$ tension (blue). Note that the range of tensions is almost a factor of two higher for these tests than for the Technica results.

Comparisons for the average values in the Tables are restricted to similar ranges in tension.

Table 3. Ascentta $90^{\circ}$ FBG, mandrel-wrap results.

\begin{tabular}{|l|l|l|l|l|l|l|}
\hline Coating & \multicolumn{3}{|l|}{ Bare } & Acrylate & Polyimide \\
\hline Mandrel Dia. & Min & Norm & Min & Norm & Min & Norm \\
\hline 0.5 in. & 17.6 & 0.2 & 59.8 & 0.2 & 18.3 & 0.1 \\
\hline $\mathbf{1}$ in. & 12.77 & 0.1 & 46.3 & 0.2 & 22.6 & 3.4 \\
\hline
\end{tabular}

In terms of developing an UID, the most useful result obtained from the mandrel measurements is that a smaller contact area seems to give a larger shape change $\left(45^{\circ}\right.$ wrap of Technica FBGs on a $0.5 \mathrm{in}$. mandrel). Beyond that, the results are scattered, particularly between different manufacturers. A check of the amount of shape change as a function of how much the peak position changed with tension did not show any obvious correlation. Also, the high breakage rate for the polyimide FBGs from Optromix indicates these are likely a poor choice for UID development. 

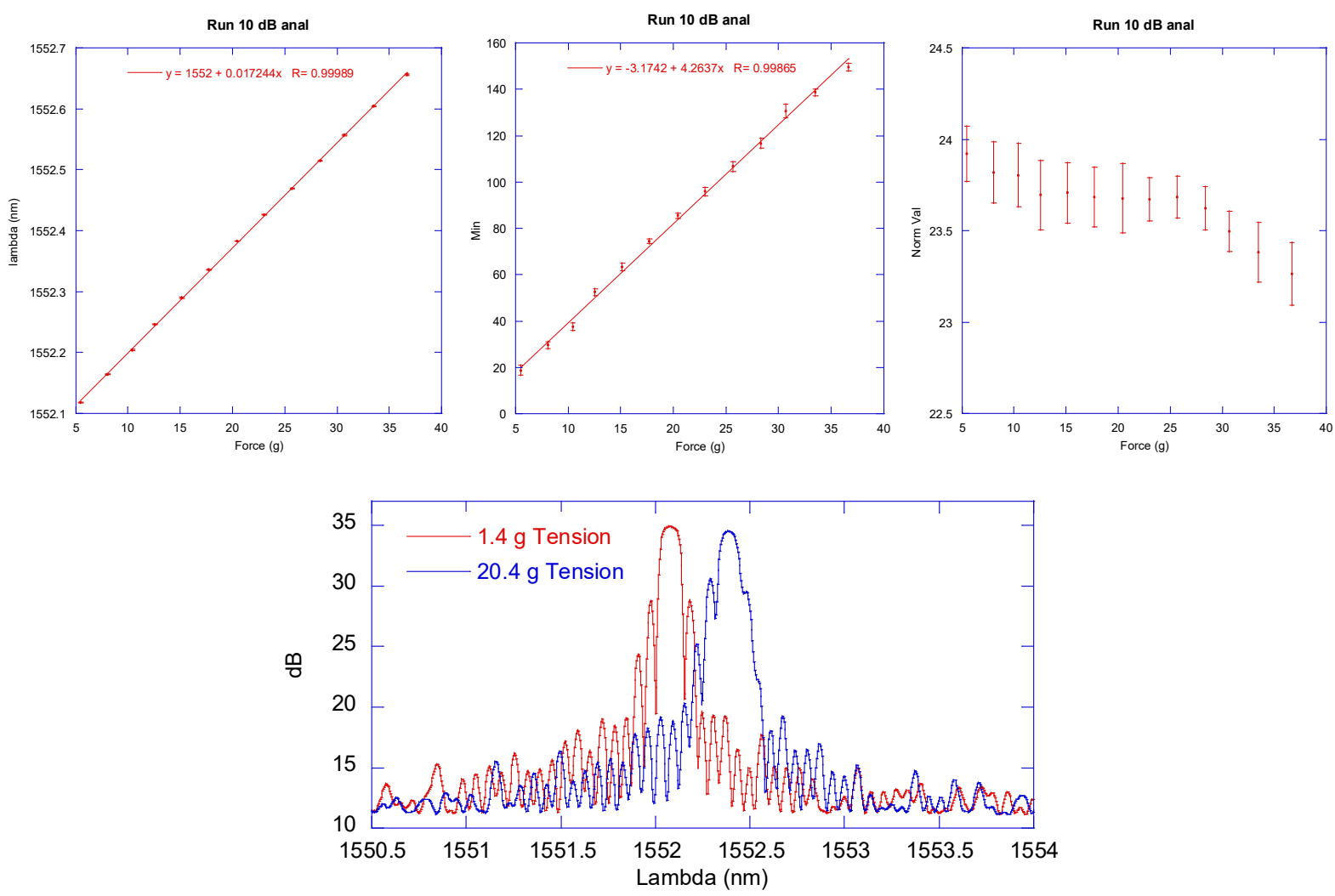

Figure 18. Results for Ascentta acrylate-coated FBG with the largest change in shape when wrapped on a 0.5 in. mandrel.

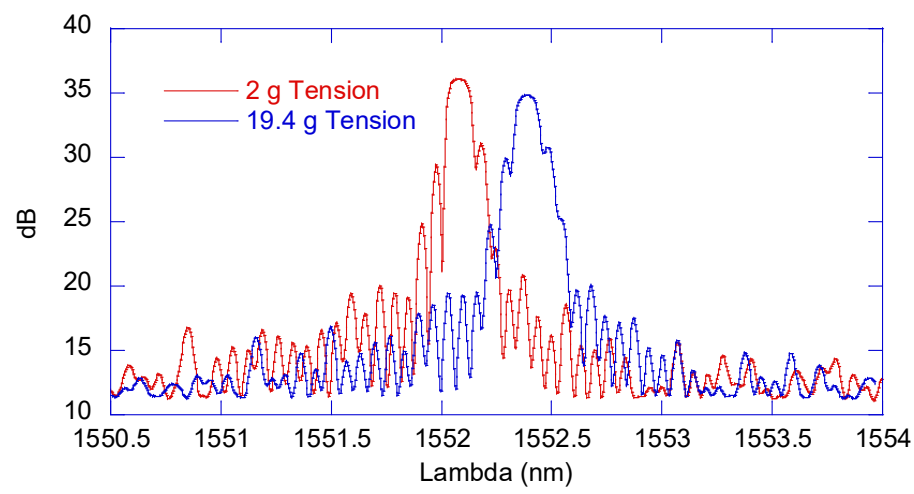

Figure 19. Change in shape for the Ascentta FBG with a large shape change wrapped $90^{\circ}$ on a 1 in. mandrel.

\subsection{DIFFERENT FBG PARAMETERS}

A series of different FBGs was obtained from Technica to explore how FBG parameters might impact the UID design. Two samples of each of the different FBGs were obtained, which allowed some comparison of how closely FBGs with the same design features matched (e.g., how faithfully could one obtain an FBG with a given design). The selected variations included a range of reflectivities from $45 \%$ to $95 \%$ in steps of $10 \%$, different FBG lengths from 2 to $10 \mathrm{~mm}$ in steps of $2 \mathrm{~mm}$, reflection widths of $0.25 \mathrm{~nm}$ and $0.5 \mathrm{~nm}$, and various wavelengths spanning the C-band. The FBGs were meant to be uniform without apodization; however, when compared to the Ascentta results, the spectra clearly indicate a significant degree of apodization was included in the devices. 


\subsubsection{Center wavelength}

The features of the different FBGs are inherent to the intensity of the index change written into the fiber core, the length of the grating, and any variations in the envelope of the intensity change (e.g., an overall Gaussian FBG modulation profile is frequently used to create an apodized reflection spectrum). All of these properties can be adjusted to compensate for the wavelength for which the FBG is designed, particularly over the relatively narrow wavelength range of the IR-C band; consequently, there are no significant variations in the FBG properties as a function of the peak wavelength. This holds true for both the simple spectra of the apodized Technica FBGs, as well as the more complex signatures of the nonapodized devices from Ascentta. This can be seen in the various spectra presented throughout this report.

\subsubsection{Reflectivity}

The reflectivity of a uniform-period FBG is due to a combination of the factors, including the length of the structure written into the core and the intensity of the index variation (contrast). For a high contrast grating, the bandwidth is a function of the contrast, but for lower contrast gratings, it is a function of both the contrast and inversely proportional to length. These parameters are selected by a manufacturer to achieve a desired FBG design. Results for FBGs with a specified bandwidth of $0.25 \pm 0.05 \mathrm{~nm}$, a length of $10 \mathrm{~mm}$ and stepped reflectivities are shown in Figure 20. The shape varies, and the nominal central bandwidth (specified as $1550 \pm 0.1 \mathrm{~nm}$ ) wanders somewhat. The change in shape is more obvious in overlaid, normalized, decibel plots where the center wavelengths have been shifted to coincide. In general, the response widens as the reflectivity is decreased, although the overall shape is somewhat rounder. It is hard to draw significant inferences about the sidelobes because they are reduced in these apodized gratings. However, in general, a range of reflectivities can clearly be used to create a complex UID spectral pattern, and relative reflectivity changes as small as 5\% should be distinguishable.
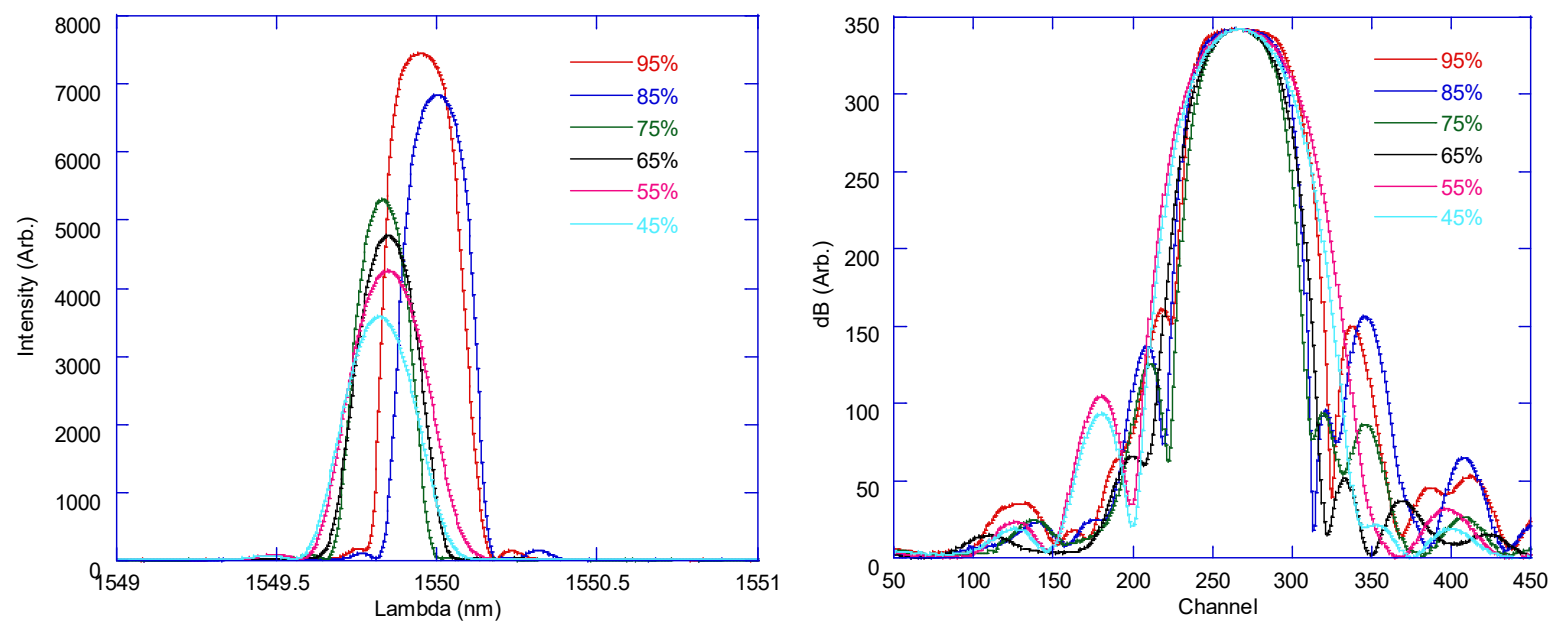

Figure 20. As measured, linear response for gratings with differing reflectivities. The plot on the right has the peaks coaligned and shows the normalized decibel response. The trend with lower reflectivity is a rounder, wider response, even though the nominal shape parameters are supposed to be the same.

\subsubsection{FBG length}

FBG lengths of $2,4,6$, and $8 \mathrm{~mm}$ were obtained with reflectivities greater than $50 \%$. As mentioned above, both the reflectivity and bandwidth can be affected by the length of the FBG. The primary difference between the different reflections of these devices is the bandwidth, which varies almost 
inversely as the FBG length increases (Figure 21). Other than this broadening, there is little to differentiate the spectra. This is an indication that for use in an UID, FBGs could be obtained over a range of widths, as might be required with a lower resolution readout device. This concept was also verified inadvertently with thermal tests of a broken, nonapodized Ascentta grating, as indicated in the section on environmental testing below.

The variation in spectral feature size as a function of FBG length is an important observation to the UID design. In particular, it means that one can tailor the spectral feature size to the resolution of a readout device so that lower resolution (i.e., less expensive) devices should be viable for this application. However, the spectral shift remains a feature of the imposed stresses so that to resolve changes in the shape of a multi-FBG UID with a lower resolution readout device implies larger stresses, or at a minimum, good line localization ability.

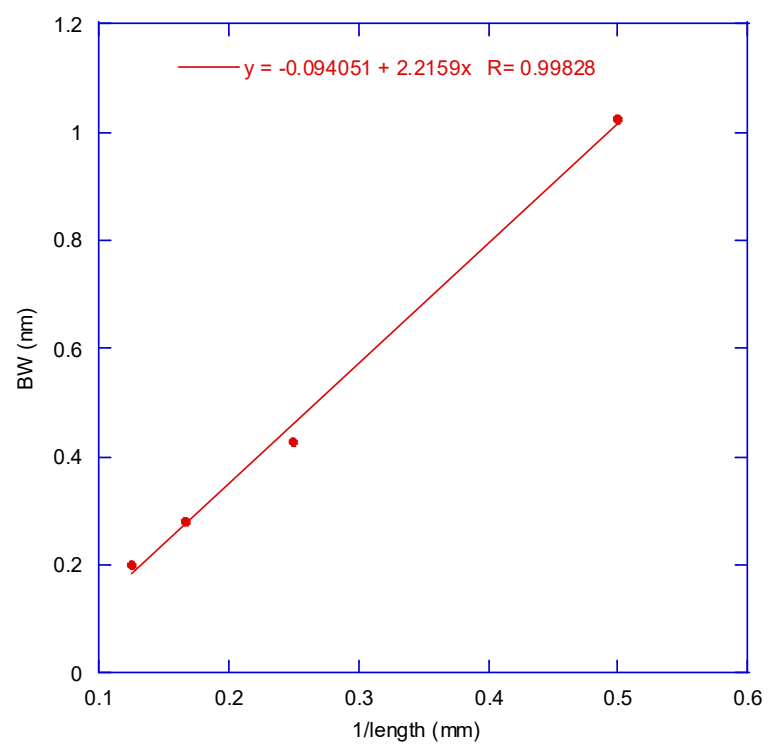

Figure 21. Plot of the bandwidth vs the inverse of the FBG length. This is almost a linear relationship.

\subsection{SHAPE DISCRIMINATION}

To explore how well the difference-correlation can distinguish between nominally identical FBGs, several FBG pairs with identical specifications were ordered from Technica. The shape of one of the pair was taken and the difference-correlation run with its own spectrum and that of its "identical" companion. The results are plotted in the left panel of Figure 22.

An independent test of shape discrimination was performed with data from the Ascentta fibers (Figure 22, right). All of these FBGs had nominally identical specifications except for the central wavelength and the coating material. In fact, the complex shapes look remarkably similar from FBG to FBG. For the comparison, the shape of each FBG was taken (using the same shape width), and the difference correlation run with the FBG spectrum itself and with the spectra of all other FBGs. Note that for the 9 FBGs, there are 36 independent pairings. The histogram does not include duplicate results, so the shape from FBG 1 run with FBG 2 is included but the shape from FBG 2 run with FBG 1 is not. (The latter value gives approximately the same value as the former one, and differences are only based on the binning, which is the reason for the nonzero autocorrelation values.) 

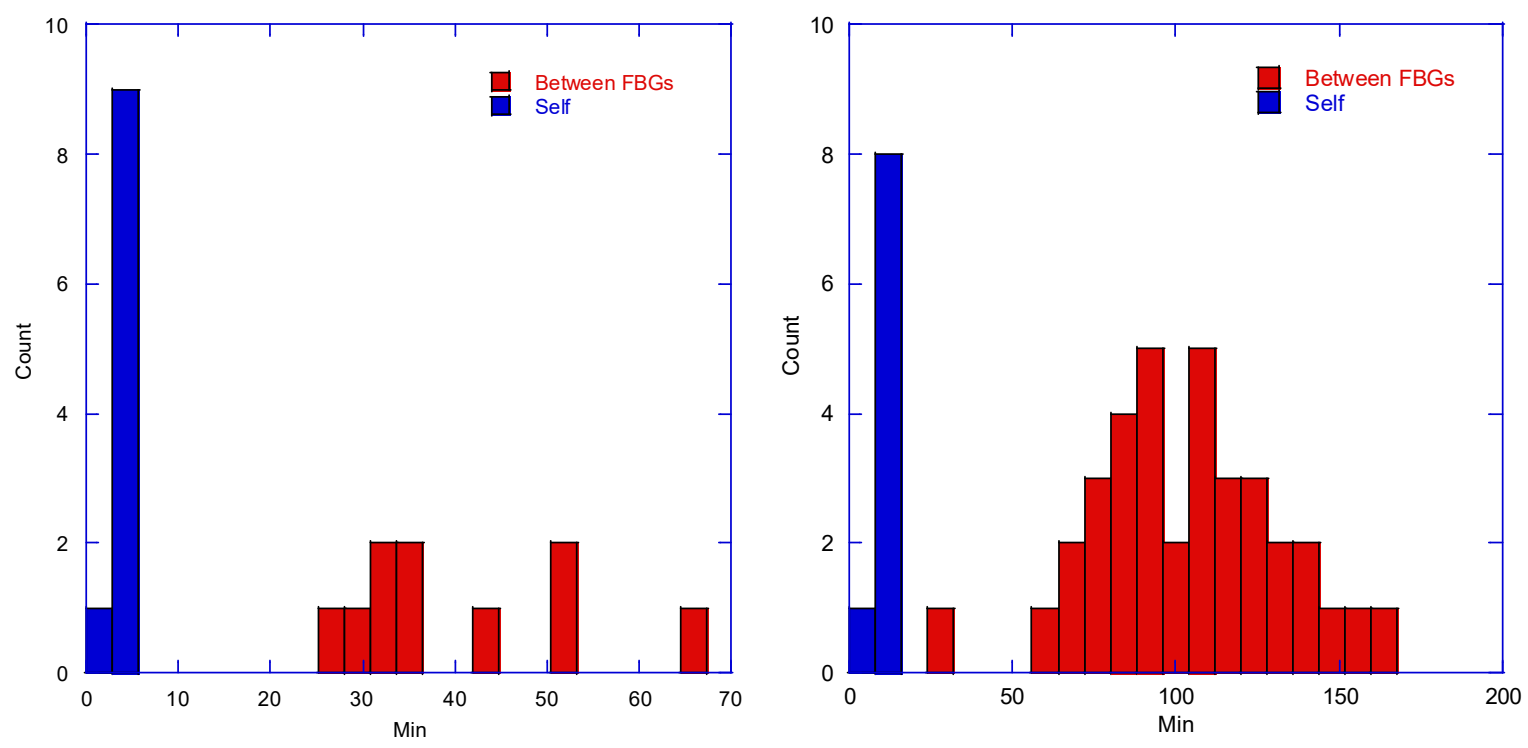

Figure 22. Histogram of difference-correlation values returned for FBGs with themselves and with others of nominally identical properties. Right, Ascentta FBGs, and left, Technica FBGs. The minimum values returned by the Ascentta FBGs are larger because a larger spectral region is used to define the reference shape because of the increased complexity of the nonapodized response.

In both histograms, there are two clear populations identified. The width of the inter-FBG results is quite broad, and with many FBGs, some overlap is likely to occur between the two sets. However, in general this appears to be a valid statistic to identify an individual FBG.

\subsection{FBG COMBINATIONS}

The concept for the UID is to include multiple FBGs in a single serial fiber. One of the questions this raises is how the signals from the different devices add. Specifically, the response from a single FBG is a consequence of the interference of the wave nature of the light propagating through the FBG. Do any interference effects persist for FBGs separated by a substantial length $(>>\lambda)$ of ordinary fiber? This was investigated by using two of the $2 \mathrm{~mm}$ long FBGs with $\sim 50 \%$ reflectivity (Figure 23). If interference effects are present, the bandwidth of the combined system should be narrower (i.e., acting more like a $4 \mathrm{~mm}$ long grating) than having two separate reflectors. The result of the inline FBG pair is clearly much wider than a single $4 \mathrm{~mm}$ grating.

To understand the response from the two combined FBGs we have developed a model that uses the combined individual response from the gratings, summing the responses incoherently but accounting for the effect of reflections to determine the light that reaches the sensor:

$$
I_{\text {ref }}=I_{0} R_{1}+I_{0} T_{1} R_{2} T_{1}+I_{0} T_{1} R_{2} R_{1} R_{2} T_{1}+
$$

The terms on the right side of the equation are as follows: The first term is the input intensity $\left(I_{0}\right)$ times the reflection coefficient of the first grating $\left(R_{l}\right)$. The second term is the light that reaches the second grating and makes it back to the sensor. This is given by reflection of the initial light that reaches the second grating (the initial light times the transmission of the first grating $\left(T_{1}\right)$, times the reflection coefficient), but is reduced by the light that is back reflected by the first grating (another factor of $T_{l}$. The third term is light from the first reflection by the second grating that is reflected back to the second 
grating and then reflected back toward the sensor (with subsequent "loss" due to transmission of the first grating. This process is repeated iteratively with less and less light reaching the sensor from each iteration.
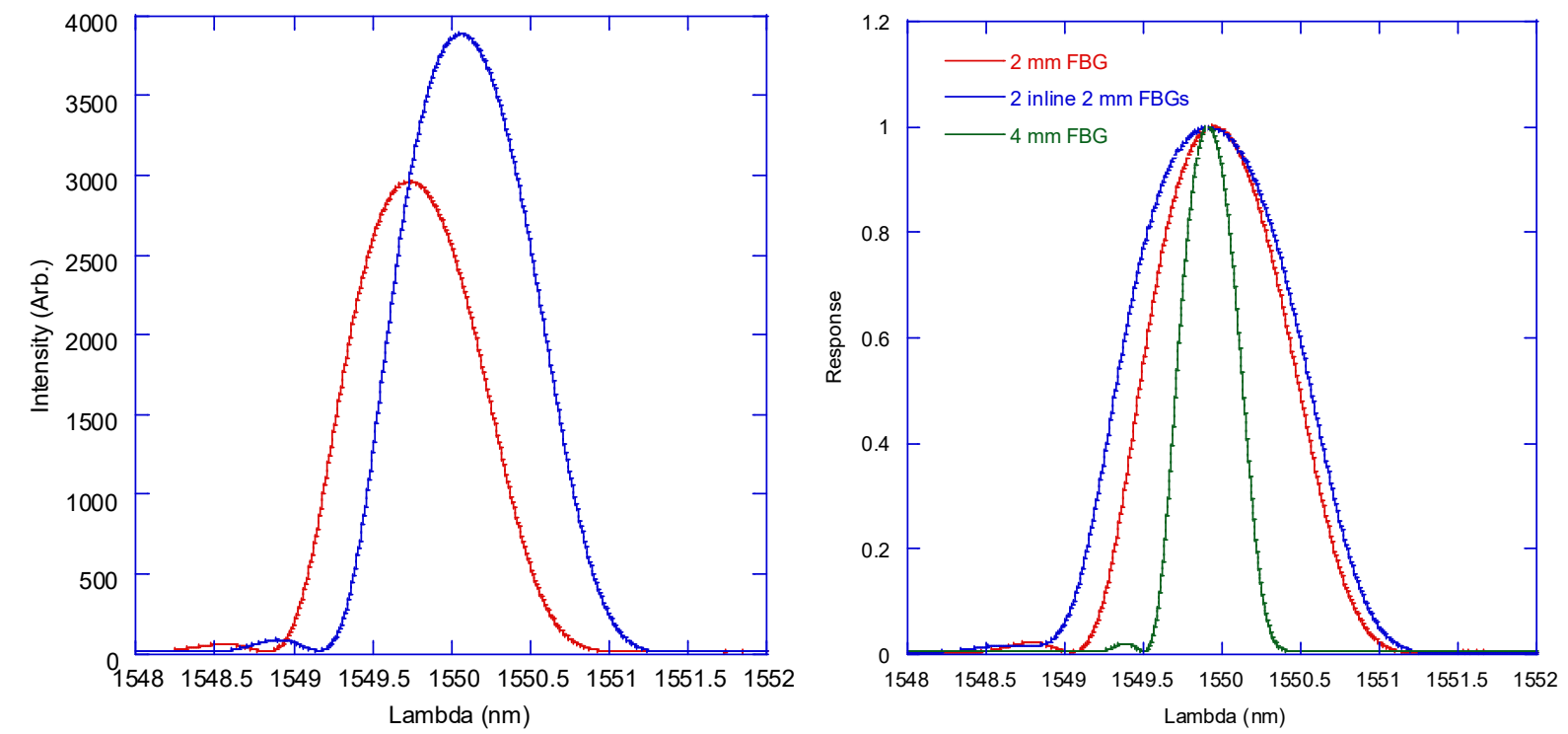

Figure 23. Individual response from separate $2 \mathrm{~mm}$ long FBGs (left). Normalized response from a single $2 \mathrm{~mm}$ long FBG, a single $4 \mathrm{~mm}$ long FBG, and the returned spectrum from the two $2 \mathrm{~mm}$ long FBGs in series on the same fiber.

Using this simple expression with the reflectivity as a function of wavelength taken from each individual FBG's response scaled to the maximum intensity seen by the interrogator used on the project with a highreflectivity FBG, and assuming that the transmission is 1-R (e.g., ignoring insertion losses of the coupler, etc.), we arrive at the results shown in Figure 24, which match very well. The slight offset in the central wavelength can be easily attributed to the fact that the data were collected on separate days without thermal control. 


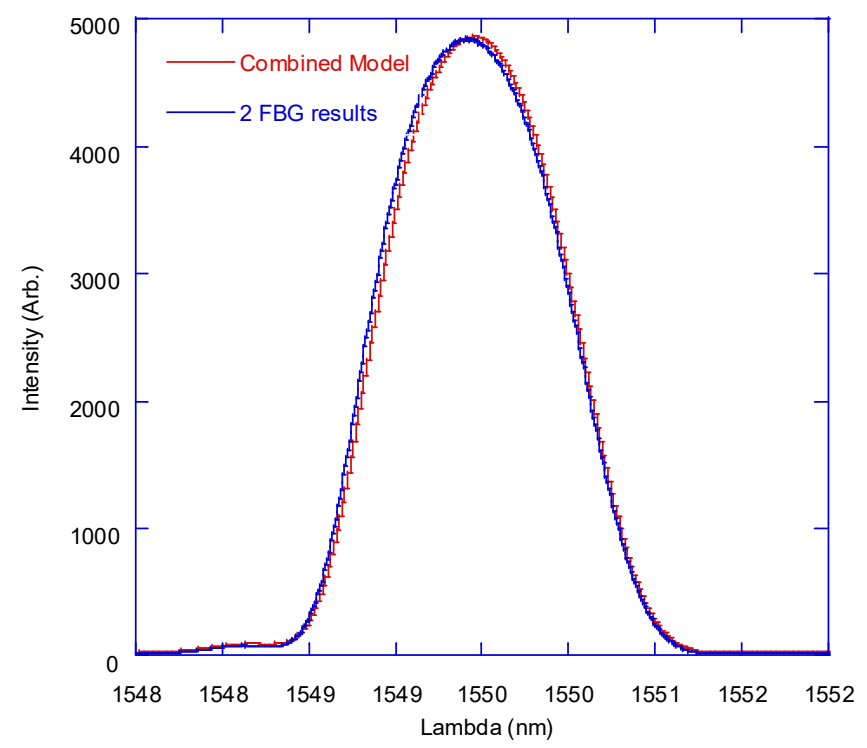

Figure 24. The results obtained from the model (2) compared to the data collected from the two FBGs on the same fiber-optic line.

\subsection{TEMPERATURE AND RELATIVE HUMIDITY}

It would seem that the simplest test that can be applied to an FBG is to monitor its response as a function of ambient temperature. Original long-term tests on potted gratings showed diurnal variations due to daily fluctuations in the temperature of the laboratory of $\sim 2^{\circ} \mathrm{C}$. Because of this level of sensitivity and the concerns about temperature changing the shape of a UID response, a thermal/humidity environmental chamber was acquired, and a series of temperature runs on different FBGs performed. For the runs, the temperature was stepped in $5^{\circ} \mathrm{C}$ increments from $-15^{\circ} \mathrm{C}$ to $75^{\circ} \mathrm{C}$, with a 0.5 hour soak at each temperature. The chamber monitored the temperature to ensure it was within a specified range of the desired temperature, with the soak time only incrementing if the temperature was within the given interval (typically $\pm 0.5^{\circ} \mathrm{C}$ ). For these measurements several FBGs with different wavelengths were sometimes positioned in series on the same channel of the optical interrogator.

The environmental and FBG data were run asynchronously with the FBG readout obtained at 5 min. intervals. Both the FBG and the Phidget temperature and humidity were read out and recorded by the same program. The environmental chamber conditions were recorded by its autonomous controller and later downloaded to the FBG host computer. To synchronize the two data streams, the FBG host computer was configured to act as a network time protocol server for the chamber. To ensure that the temperature was at the set point for the analysis, only those points within $0.5^{\circ} \mathrm{C}$ and within $1 \%$ relative humidity (if controlled) of the set point for the chamber at the time the data were recorded were used.

\subsubsection{Technica temperature and humidity}

Results for four different FBGs from Technica (two of each coating type) are given in Figure 25. The results for the FBGs in the bottom row show greater nonlinearity than those in the top row, particularly at the lowest temperatures. This was eventually traced to variations in humidity as the temperature changed (Figure 26) during the thermal run. This is an indication that the fiber coating is absorbing water from the air and presumably swelling slightly [8].

To verify this was the problem, the chamber was run at a fixed temperature $\left(45^{\circ} \mathrm{C}\right)$, and the humidity varied from $20 \%$ to $95 \%$ in $5 \%$ steps with soak times of $30 \mathrm{~min}$. As with the temperature runs, the soak 
time only incremented if the humidity was within a defined interval around the desired value (typically $\pm 1 \%$ ). In addition, a cut was applied during the analysis that required the temperature and humidity to be within $0.5^{\circ} \mathrm{C}$ and $1 \%$ of the chamber set points, respectively. The humidity results for the same FBGs given in Figure 25 are shown in Figure 27.

The variation of the peak spectral return with humidity for the two acrylate-coated FBGs is significantly smaller than for the polyimide-coated gratings. In fact, the variation over the entire humidity range for the acrylate-coated FBGs is comparable to the variation for $1{ }^{\circ} \mathrm{C}$ in temperature change and may result from systematic temperature variations across the chamber. Even for the polyimide-coated FBGs, it is only a factor of 1-3 larger.

Turning to environmental effects on the shape, as revealed by the minimum value of the difference correlation, (Figures 28 and 29) shows larger variations for the polyimide-coated FBGs than in those with the acrylate coating. This is presumably a sign that there are inherently greater stresses occurring in FBGs with the polyimide coating. This is true for both the thermal and humidity runs, and may indicate better adhesion of the coating to the fiber, which could induce local nonlinear strains on the FBG, causing the spectral shape to change (Figure 30). This could be an important consideration for creating the UID as strains may be better transmitted from the potting materials to the FBGs with better fiber/coating adhesion. 

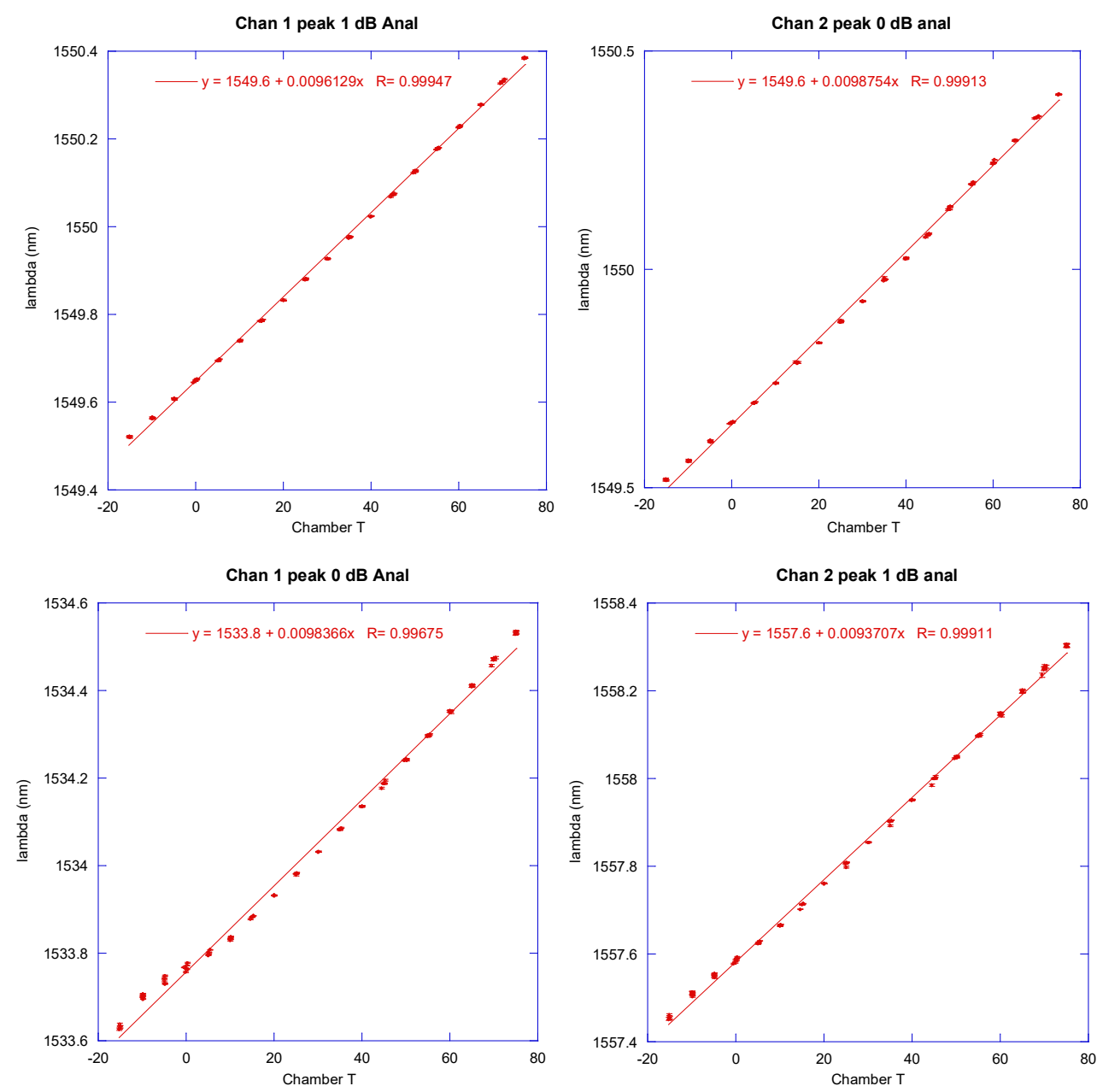

Figure 25. Change in peak wavelength as a function of temperature for four different FBGs. The FBGs in the top row were coated with acrylate, and those in the bottom row used polyimide.

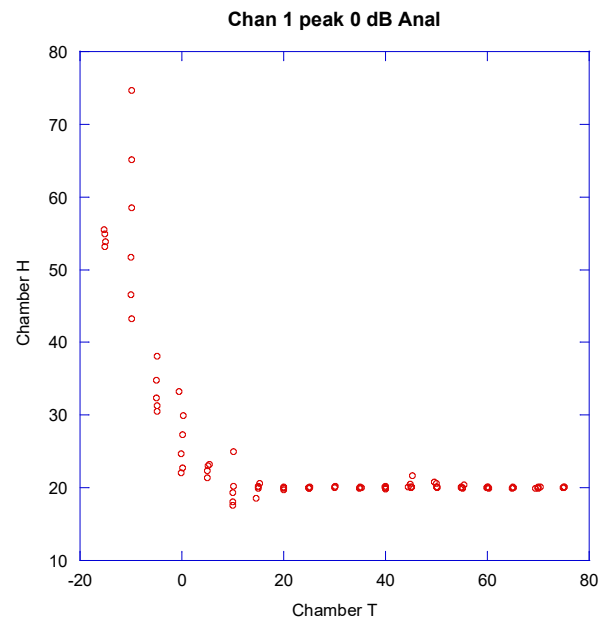

Figure 26. Chamber relative humidity as a function of temperature for the thermal runs shown in Figure 25. 

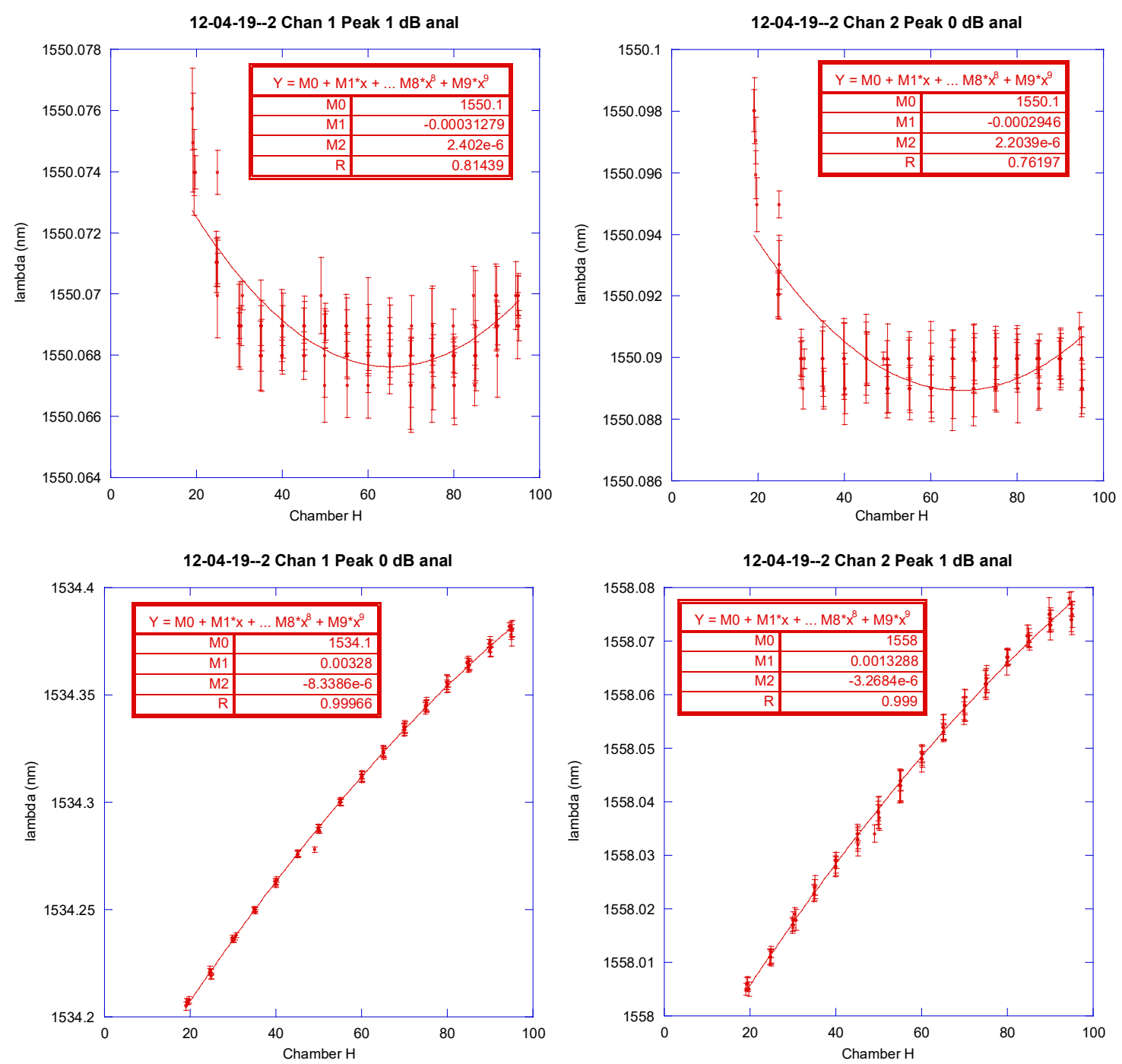

Figure 27. Changes in peak wavelength as a function of relative humidity for the four FBGs in Figure 25. The temperature was $45^{\circ} \mathrm{C}$ for these tests. The values are arbitrarily fit to a second order polynomial. The FBGs in the top row were coated with acrylate, and those in the bottom row used polyimide. 

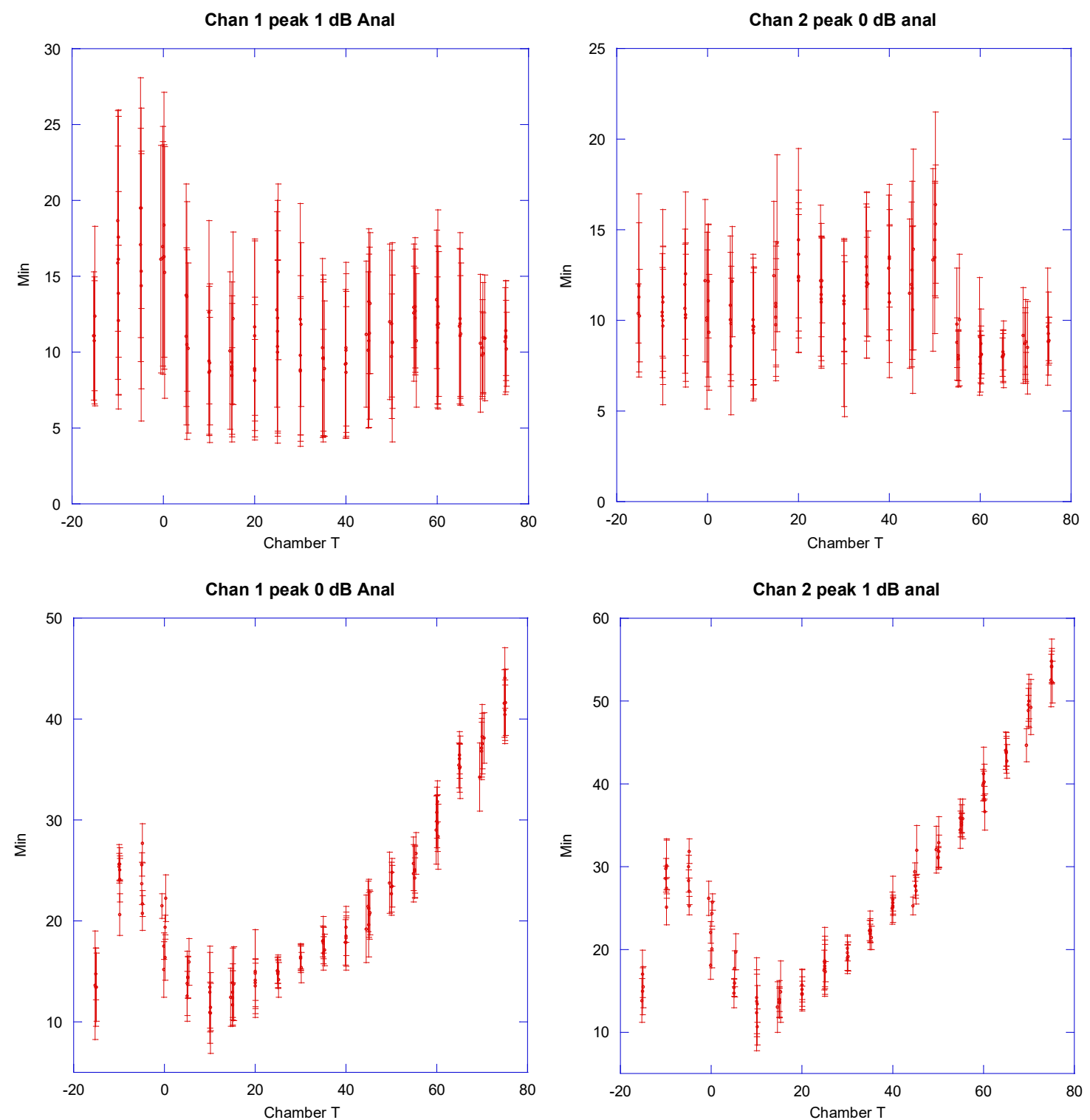

Figure 28. Difference-correlation values for the Technica FBGs for the thermal run. The FBGs in the top row were coated with acrylate and show no change in shape during the tests. Those in the bottom row were coated with polyimide and show modest changes in shape. 

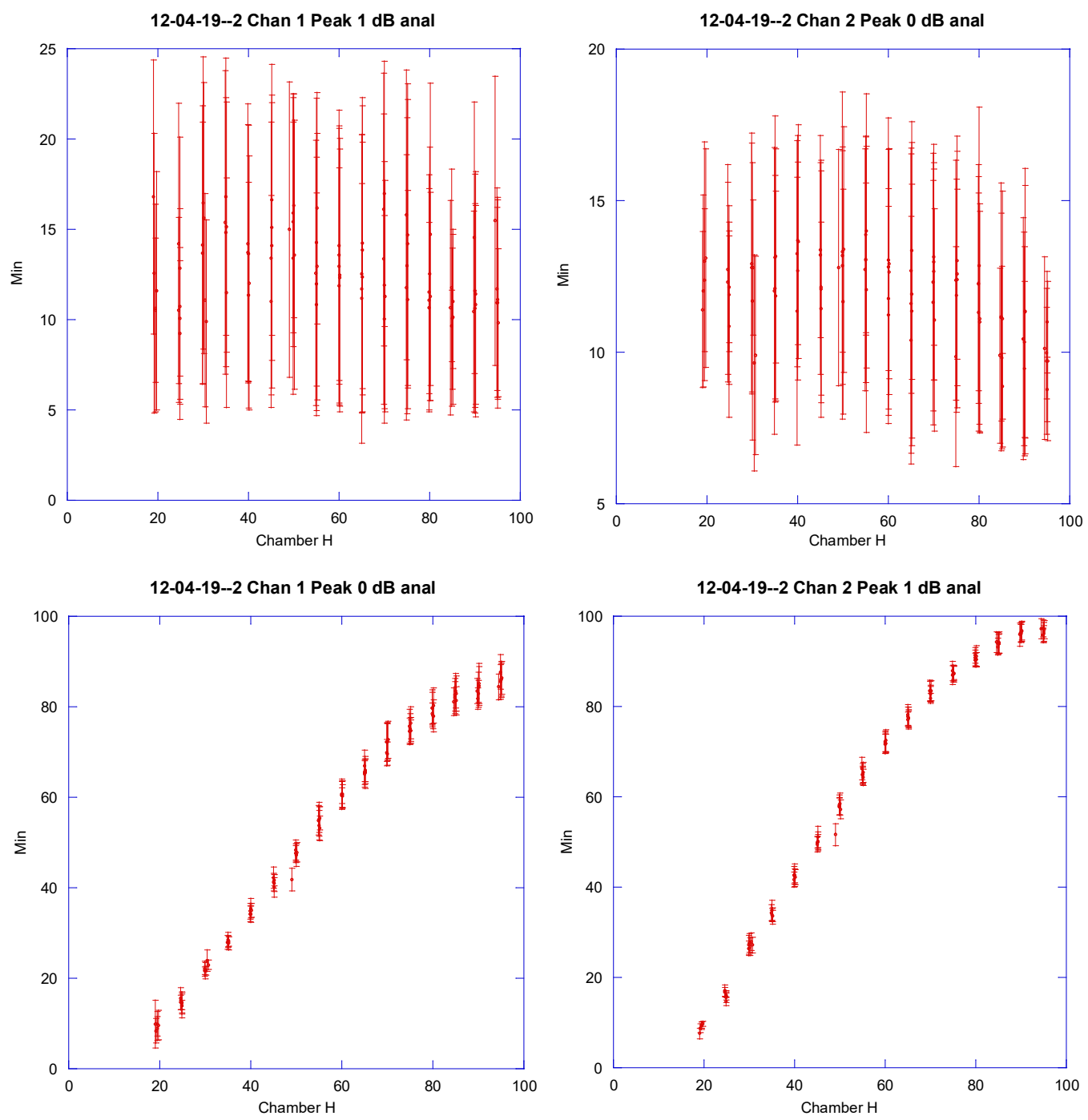

Figure 29. Difference-correlation value for the Technica FBGS for the humidity run. The FBGs in the top row were coated with Acrylate, while those in the bottom row used Polyimide.
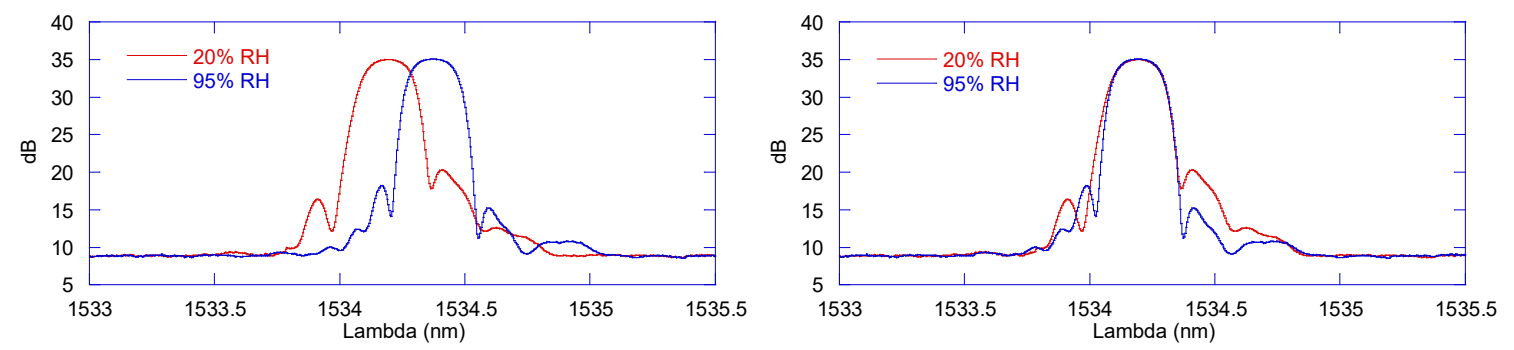

Figure 30. Change in FBG spectral response for a Technica device at two different relative humidities (left). In the right plot, the $95 \% \mathrm{RH}$ response has been shifted by $0.18 \mathrm{~nm}$ to match the peak spectral response, allowing better visualization of the shape change. This FBG corresponds to the lower left plot in Figure 29, above. 


\subsubsection{Optromix temperature and humidity}

The environmental results obtained with one of the acrylate-coated Optromix FBGs were similar to those observed with FBGs from the other vendors. There was very little change in the wavelength with humidity $(0.011 \mathrm{~nm})$, all of which occurred above $60 \%$ relative humidity (Figure 31 ) and may indicate that condensation or other factors were present. There was no change in the minimum value (e.g., the shapes remained unaffected). We note that the Optromix FBGs were apodized, so they showed minimal spectral complexity.
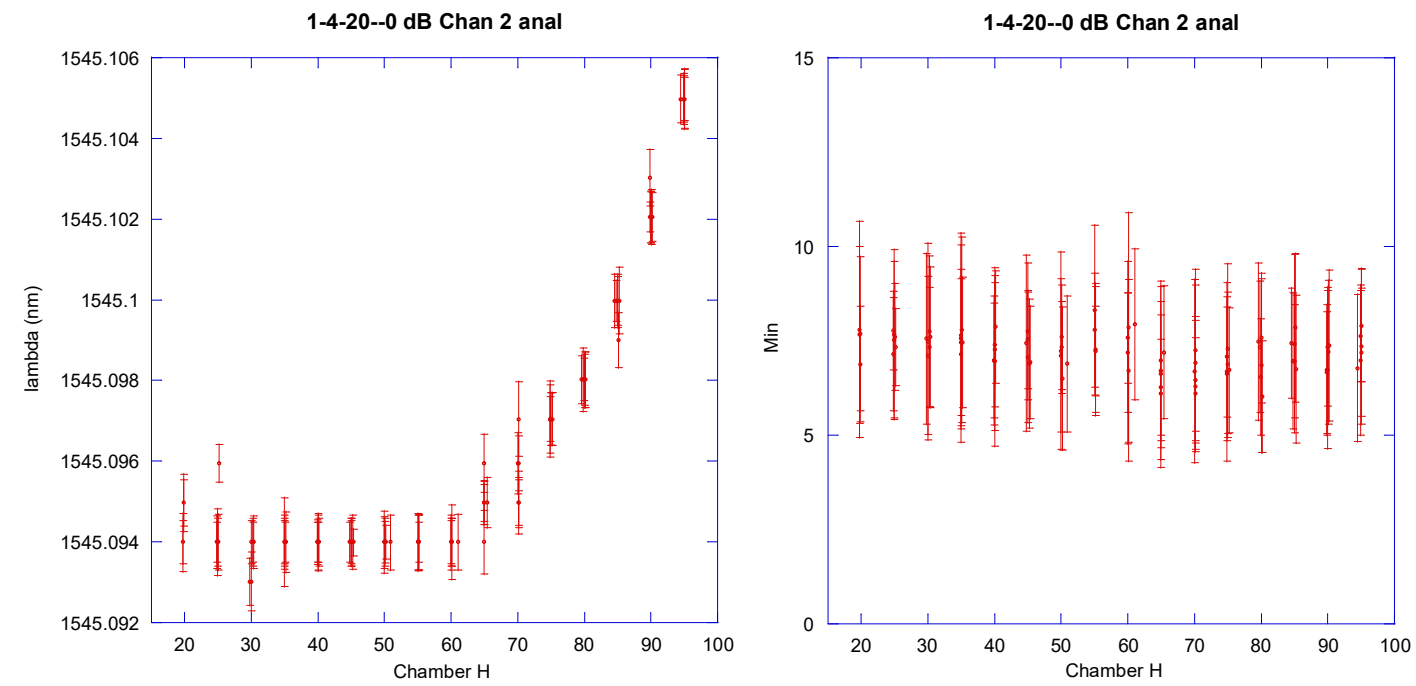

Figure 31. Humidity results for one of the Optromix FBGs. The source of the small linear shift with temperature (left) starting at $\sim 65 \%$ is unknown. There is no change in the correlation value with humidity (right).

The thermal behavior showed small deviations from linearity and changes in the shape, although these were not well correlated with the humidity changes (Figure 32).
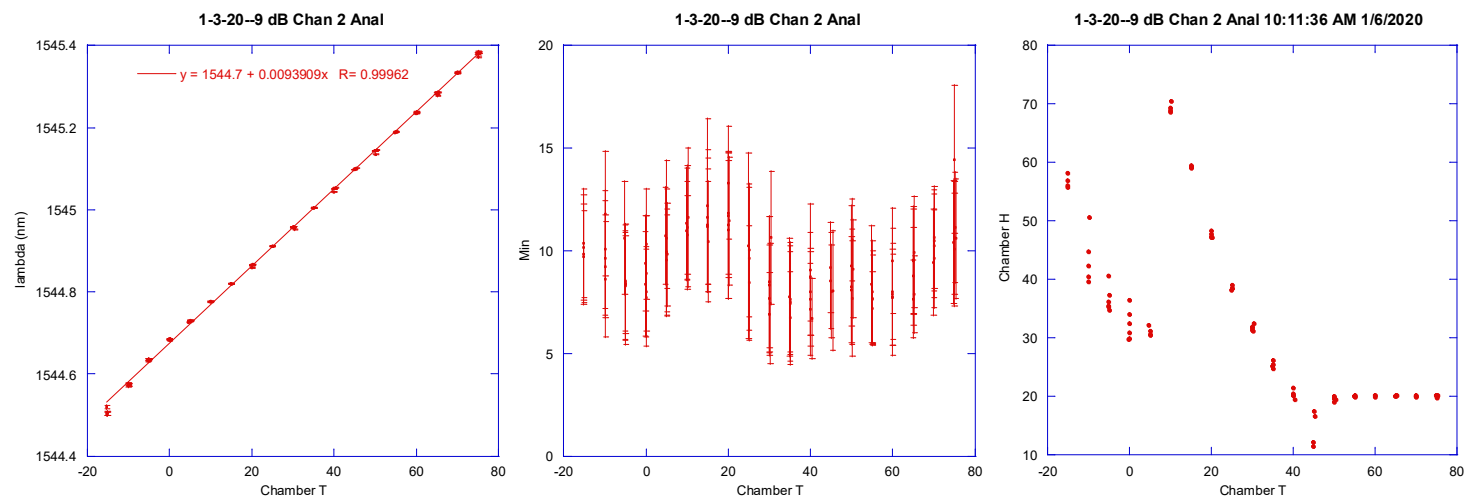

Figure 32. Temperature results for an acrylate-coated FBG from Optromix. The small changes in the shape (center) are only marginally correlated with the humidity changes-particularly to the abrupt jump at $\sim 15^{\circ} \mathrm{C}$.

Unfortunately, all three polyimide-coated FBGs from Optromix were damaged during the mandrel tests described above. A partial FBG was evaluated in the environmental chamber (Figure 33), and it showed a similar slope in nanometers per degree Celsius $\left(9.4 \times 10^{-3}\right.$ vs $\left.9.3 \times 10^{-3}\right)$ compared to the thermal tests of 
the acrylate-coated device from this vendor. It also showed a linear humidity response (slope $2.9 \times 10^{-4}$ $\mathrm{nm} / \% \mathrm{RH})$.
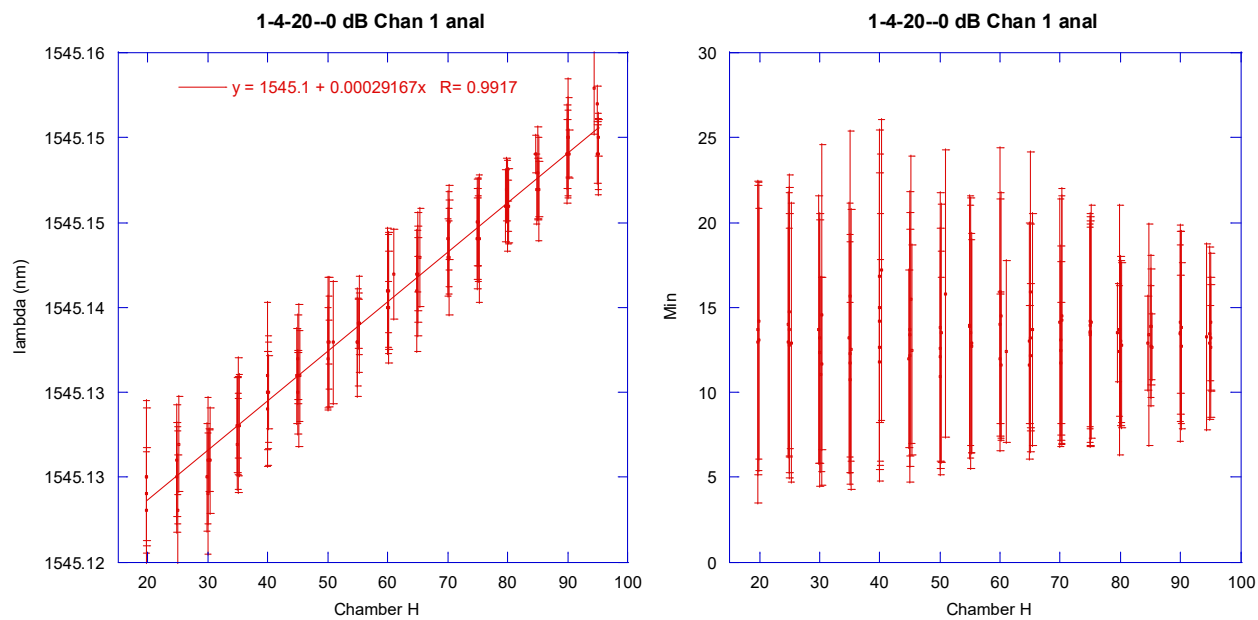

Figure 33. Humidity response of a partial Optromix polyimide-coated FBG.

\subsubsection{Ascentta temperature and humidity}

Thermal and humidity data obtained on Ascentta FBGs are shown in Figures 34 and 35, respectively. Unlike the Technica FBGs, there is little difference in the response between the acrylate and polyimide coatings. The spectral shape parameter varied from 7.5 to $\sim 20$ (compared to the delta of $\sim 70$ for the Technica FBGs). However, this can be traced to a very poor polyimide coating. It was very thin and not always present over the full FBG. The low response to relative humidity is at least qualitatively in agreement with [8], which showed that the response is directly proportional to the thickness of the coating. The small linear shift in humidity response for the acrylate-coated fiber is similar in magnitude to that from the Technica FBGs and could easily be due to small thermal shifts within the chamber. The acrylate coating from both manufacturers were of comparable thickness, but the ones from Technica were not as uniform. For neither the thermal nor the relative humidity response was there much change in the shape for this coating type, despite the spectral complexity of the unapodized design (Figure 36).
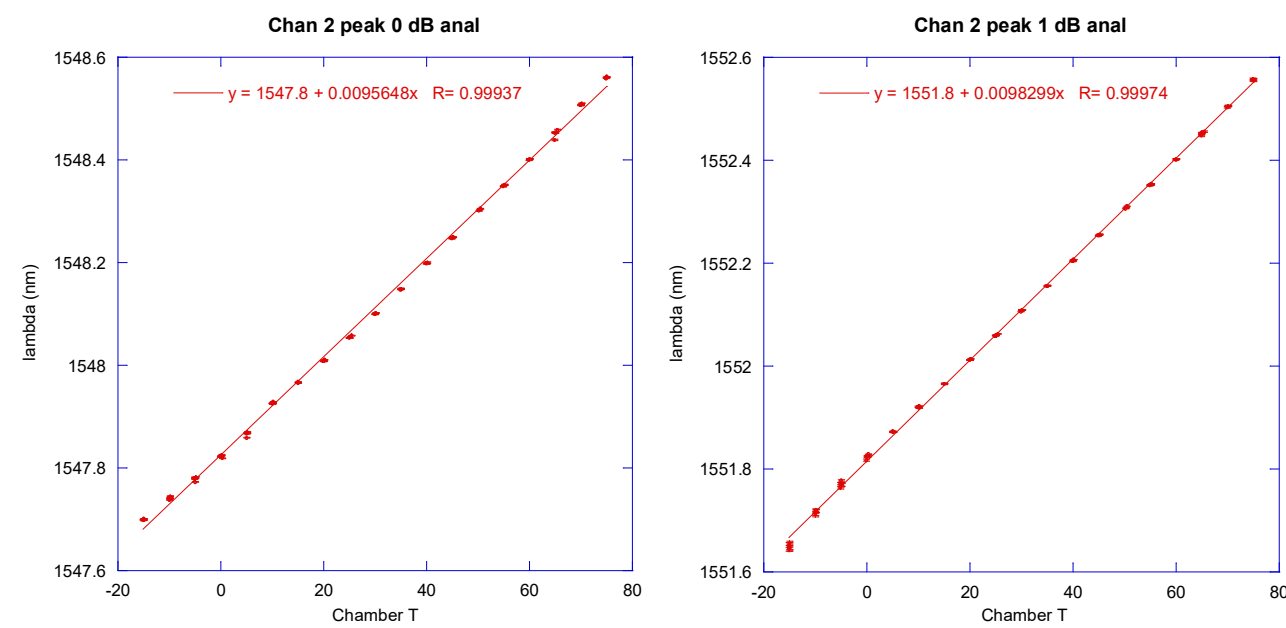

Figure 34. Thermal tests for two Ascentta FBGs. The left one has a polyimide coating, and the one on the right has an acrylate coating. 

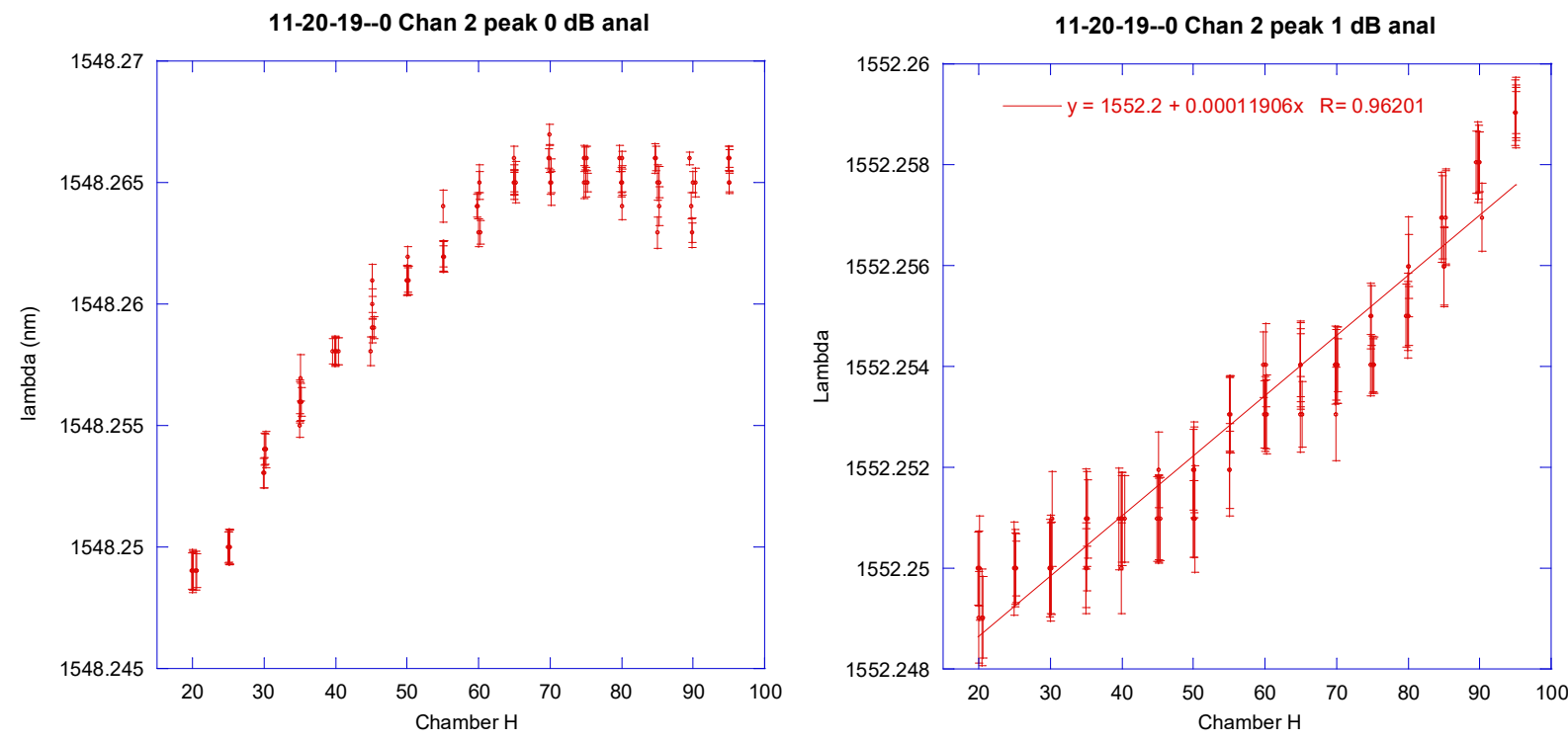

Figure 35. Humidity tests for two Ascentta FBGs. The left one has a polyimide coating, and the one on the right has an acrylate coating; however, the acrylate coating is very thin if present at all.

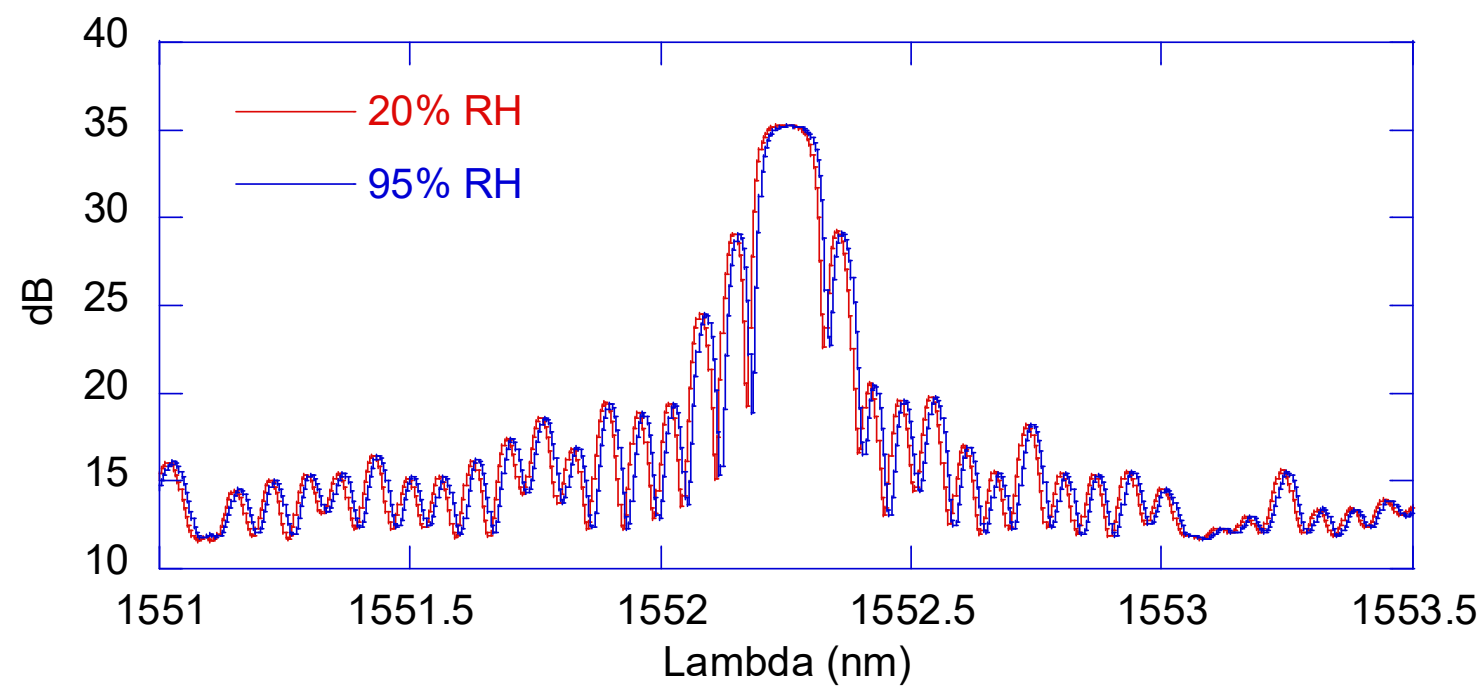

Figure 36. Change in the Ascentta polyimide coated-FBG spectral return. Despite the significantly greater spectral complexity compared to the Technica FBG, there is very little change in the spectral shape.

In addition to the recoated FBGs, three of the Ascentta FBGs were obtained bare (e.g., without reapplication of any coating after the FBG was "written"). Unfortunately, that makes the devices fairly fragile, and the one used in the environmental sample broke between the humidity and thermal runs. The relative humidity results show very little change (Figure 37). Temperature data were collected with the partial FBG that remained connected to the interrogator. The spectra returned by the original grating and the two parts after the break are shown in Figure 38. The change in width of the returned spectra are in proportion to the length of the gratings, and this agrees with basic theory and data collected with different length gratings obtained from Technica described above. 


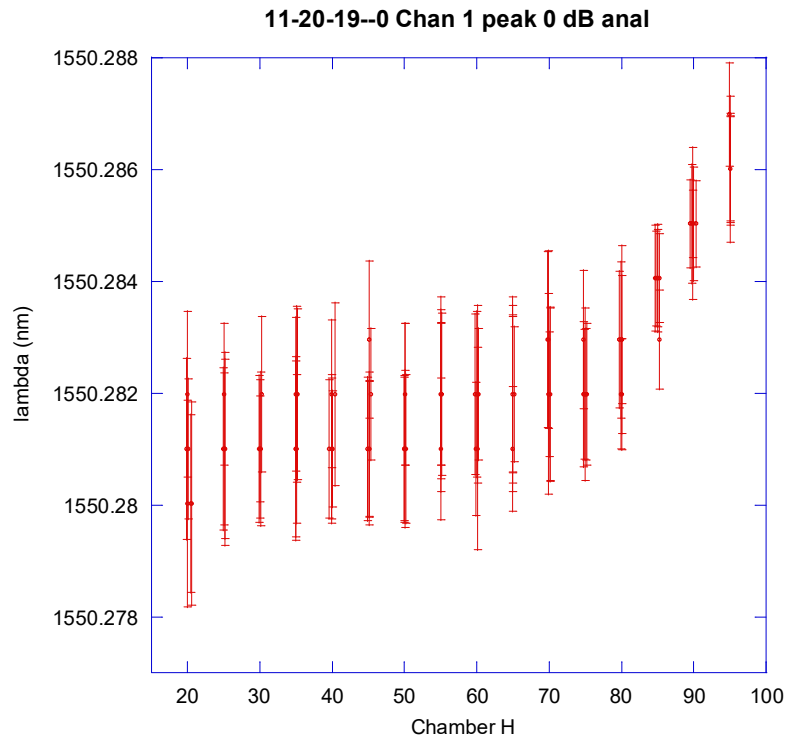

Figure 37. A Bare Ascentta FBG shows only about half the change over the full humidity range of the acrylate-coated FBGs.
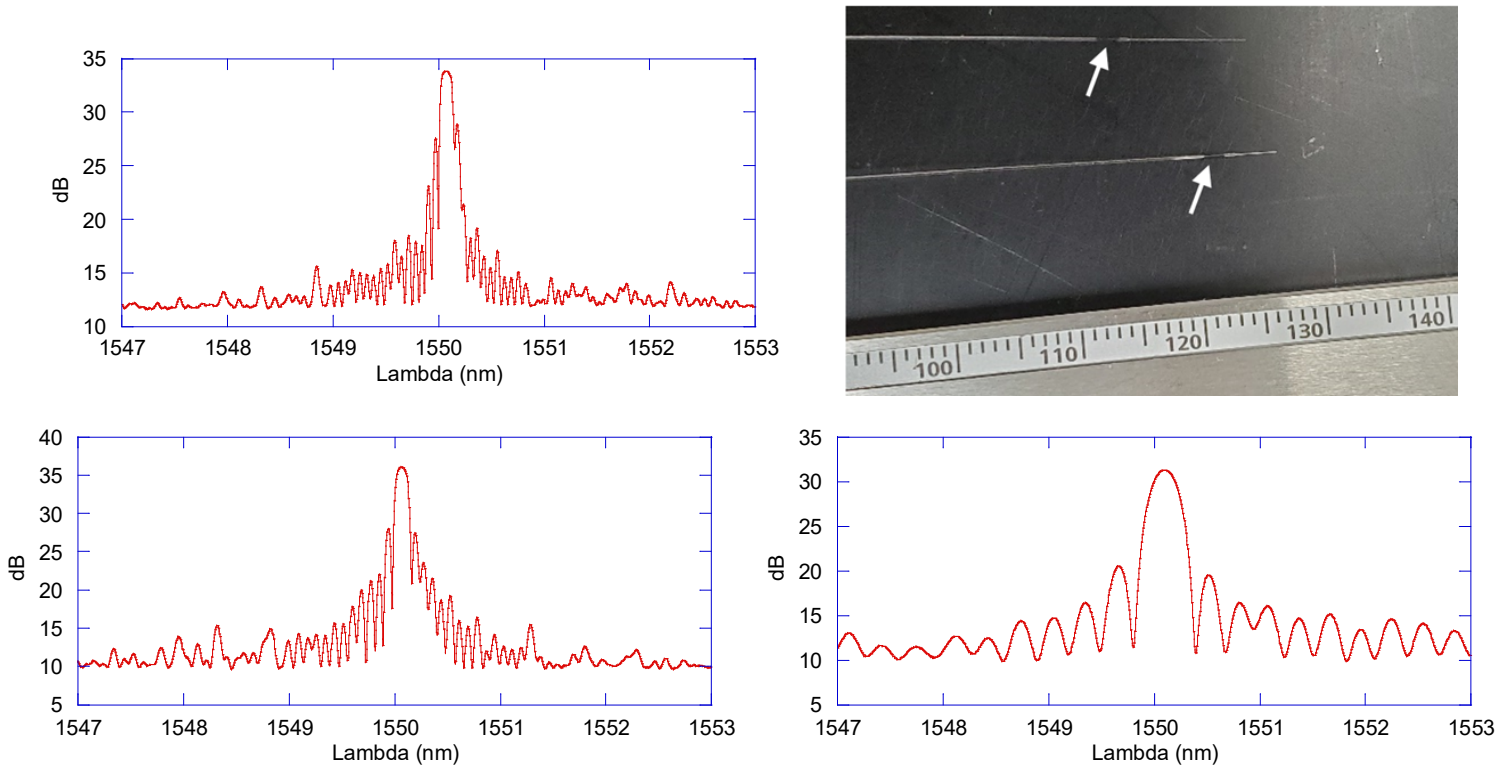

Figure 38. The spectra returned from the unbroken FBG (top, left) and a photograph showing how the FBG broke (top, right). The black marks (white arrows) on the fiber indicate the ends of the FBG. Bottom, left, the spectrum is returned from the fiber on the top of the picture and illustrates how changes in FBG length changes the width of the spectral features.

\subsection{POTTING TESTS}

To indicate tampering, the UID concept makes use of a randomized stressor field applied to a set of FBGs to create a unique signature that would be very difficult to recreate. To test this concept, a number of tests were conducted where FBGs (and some of the broken FBG portions) were potted in polyurethane glue 
that expands about three-fold on setting, polyurethane glue that does not expand, and a 5 min. epoxy that is also dimensionally stable on setting.

Only a few tests were conducted using potting compounds that do not change volume on setting, and as expected, they show only small changes to the wavelength and shape parameters (Figure 39) as the compounds set. In contrast, initial tests with the three-fold expanding compound resulted in large changes to the shape parameter (Figure 40). These changes occurred over long time periods (days) and then seemed to settle into diurnal variations due to small changes in the environmental conditions in the laboratory. These first tests were conducted on Technica polyimide coated FBGs and had similarly large changes in the shape parameter (to values of several hundred in the decibel encoded spectra).

Unfortunately, due to data acquisition issues, only one of the three tests had continuous coverage over the period as the shape evolved. Nevertheless, the results validate that shape changes can be induced through the use of potting compounds that change volume.
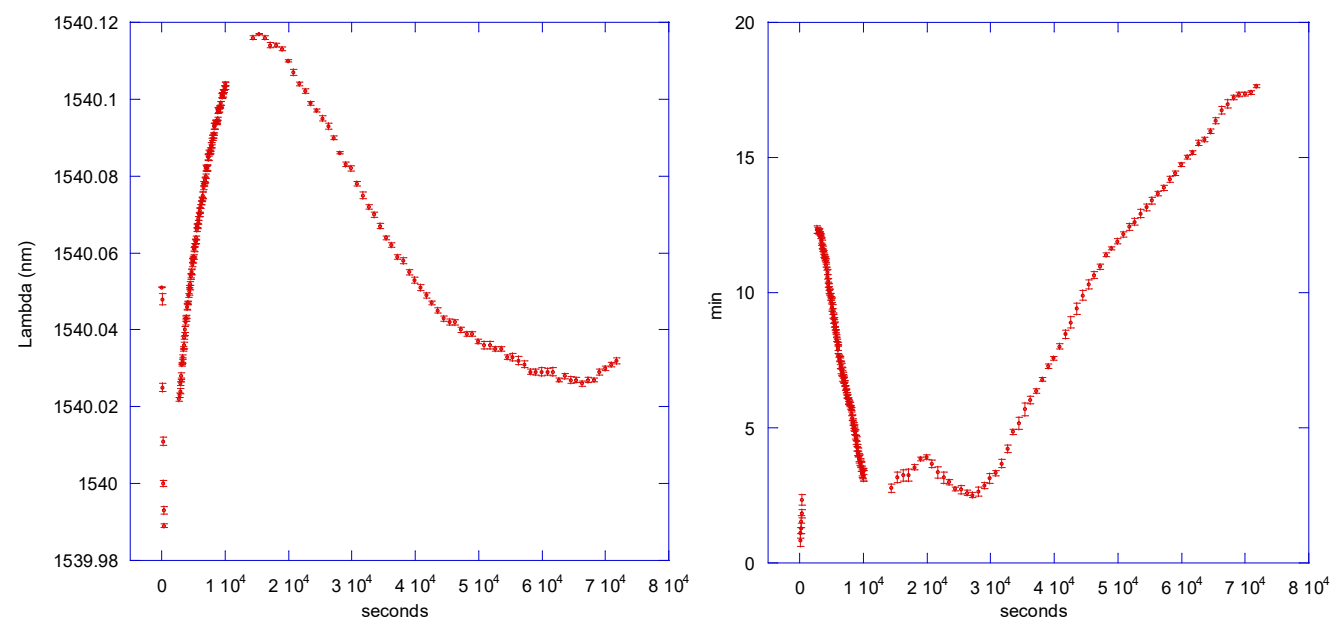

Figure 39. Results obtained on potting a Technica polyimide-coated FBG in volumetrically stable polyurethane adhesive.
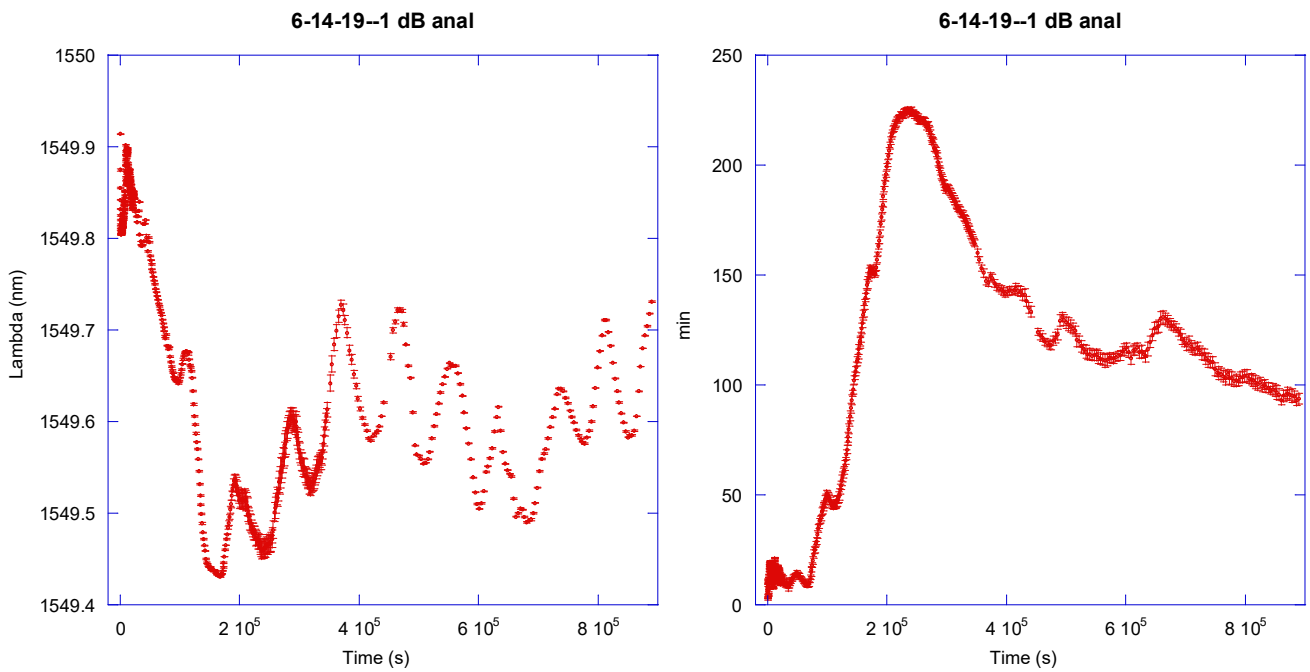

Figure 40. Results obtained on potting a Technica polyimide-coated FBG in expanding polyurethane adhesive. The shape change peaks at $\sim 2.75$ days and is significantly larger ( 200) than changes seen in the volumetrically stable adhesives ( 20$)$. 
This clear result was confounded by latter tests that used the same procedures but were conducted on FBGs written into fibers coated with acrylate (Figure 41). Those showed much smaller changes to the shape. To further obfuscate the results, a third test conducted at the same time on a polyimide FBG but potted into an array of rubber pins (Figure 42) also showed only small changes in the shape parameter.
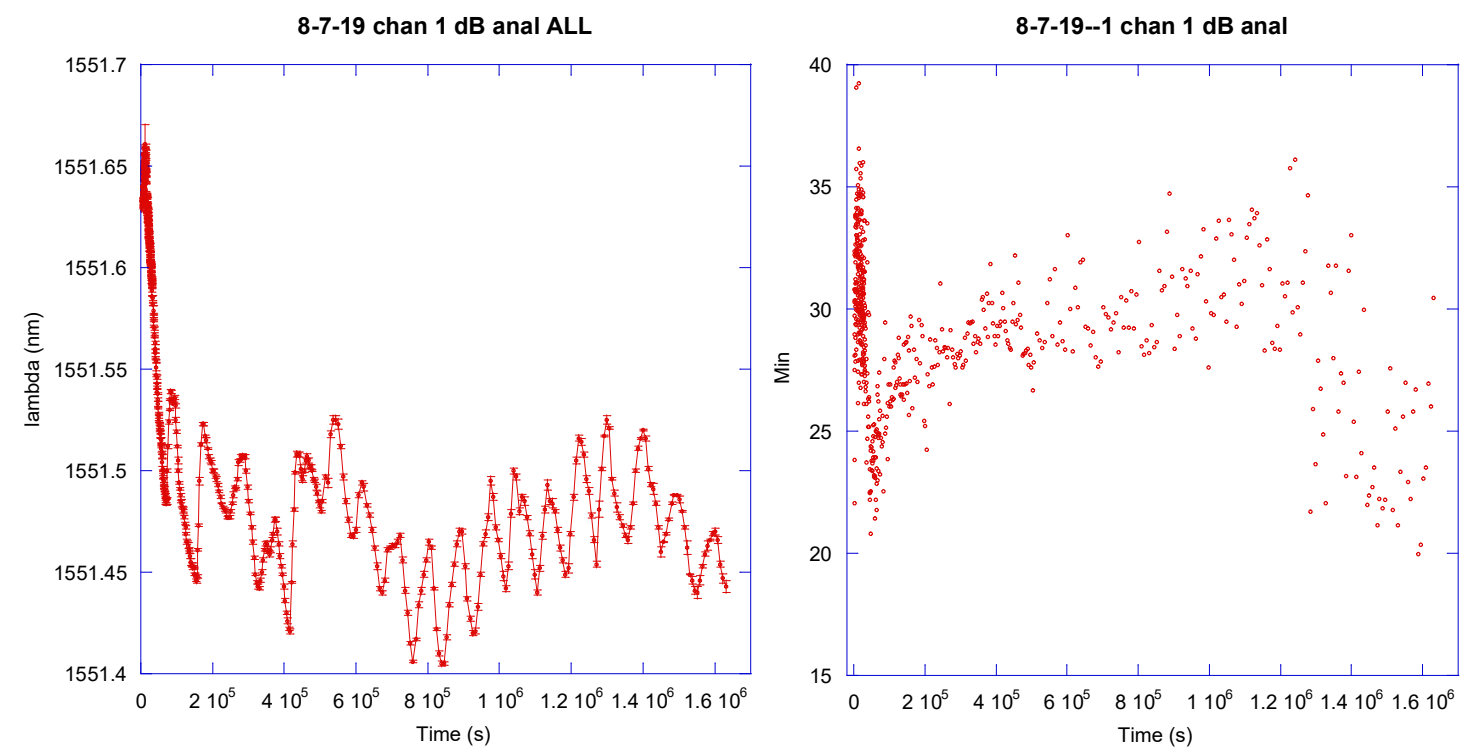

Figure 41. Results obtained with Technica acrylate-coated FBG potted in expanding polyurethane adhesive.
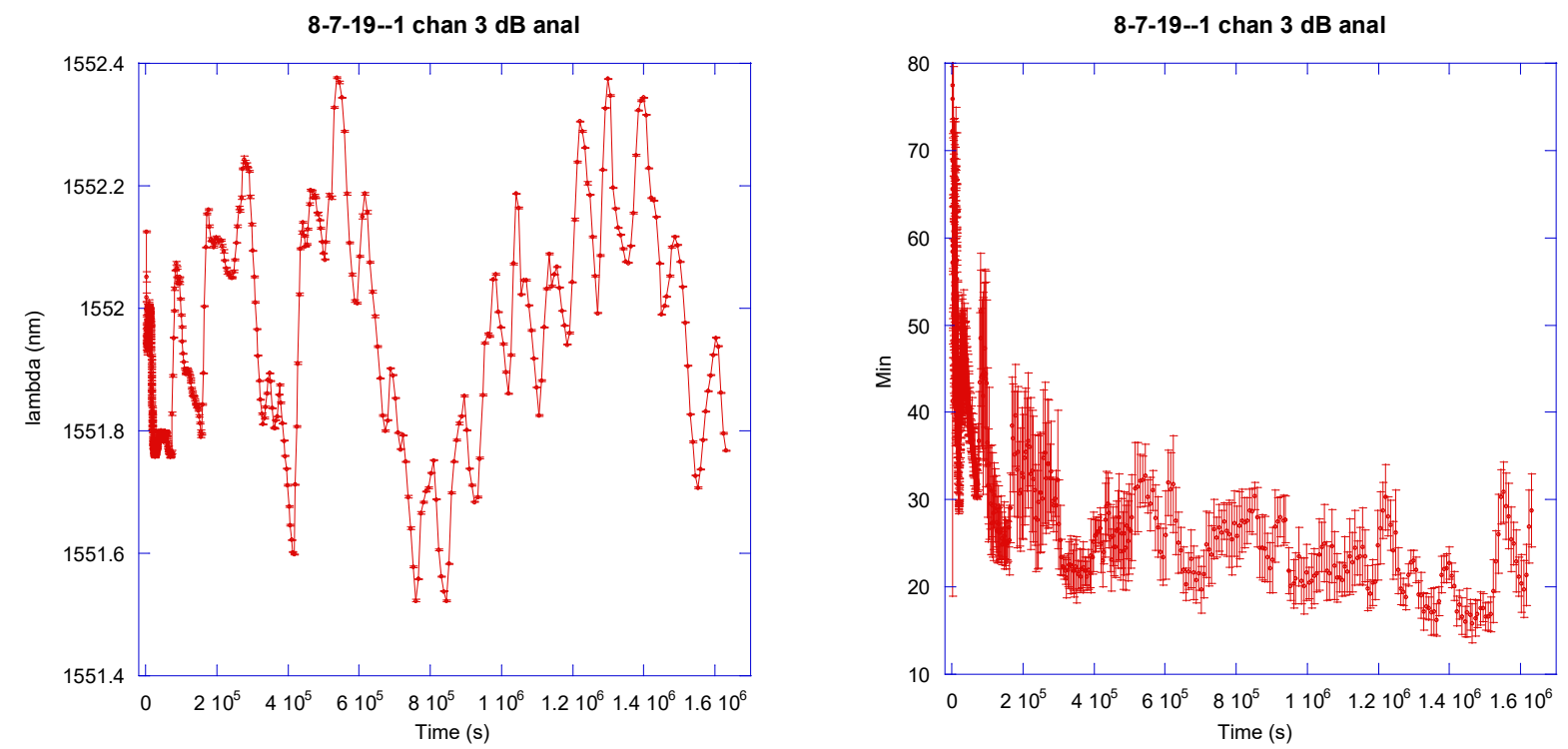

Figure 42. Results obtained with Technica acrylate-coated FBG potted into a form with rubber fingers using an expanding polyurethane adhesive.

Later potting tests using the expanding and nonexpanding adhesives on the two sides of a bare (not recoated with either acrylate or polyimide after the FBG was written) FBG broken near its center showed results similar to the initial tests. The expanding adhesive created large changes in the shape, but the volumetrically stable adhesive ( 5 min. epoxy) showed much smaller changes (Figure 43). 

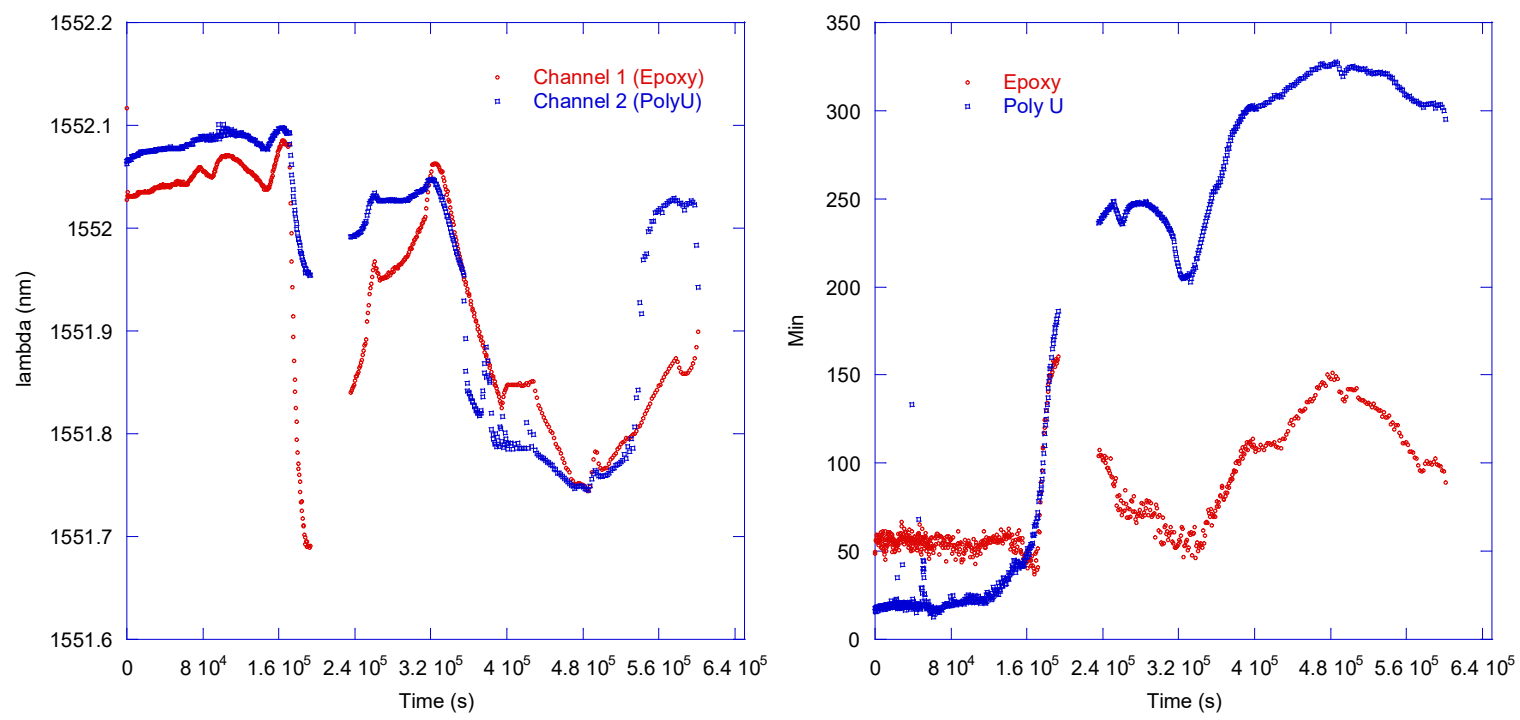

Figure 43. Results obtained with two halves of a bare FBG potted in expanding polyurethane (blue) and 5 min. epoxy (red). The overall morphologies, except for the large increase in the minimum of the polyurethanepotted FBG follow the changes in environmental conditions shown in Figure 44.

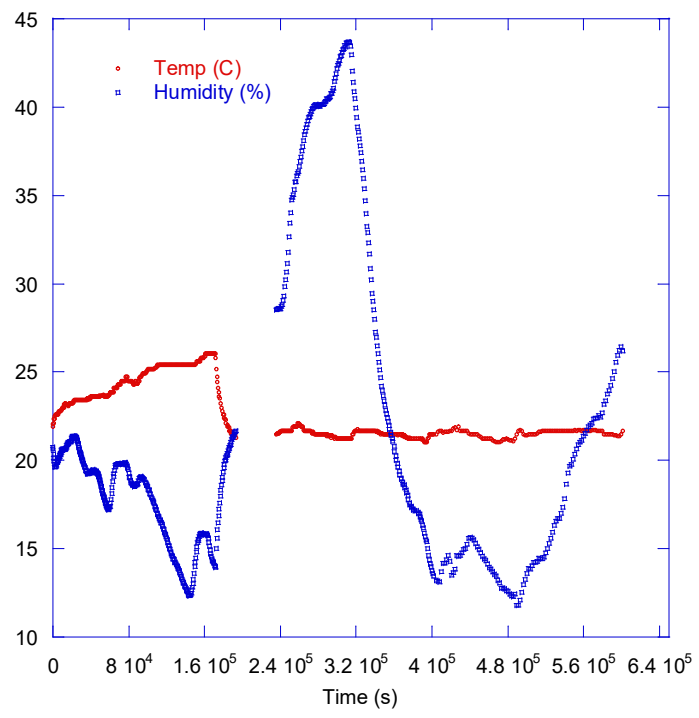

Figure 44. Changes in the environmental conditions during the test results of Figure 43.

The last results rule out that the adhesives had deteriorated and suggests that the coating on the FBG plays an important role. The hypothesis is that the acrylate coating acted as a buffer, relieving some of the stress induced on the FBG in the intermediate tests. This stress relief is not present in the bare FBGs and indicates that the coupling between the polyimide coatings and the FBGs in the original tests was stronger than for the acrylate coatings. The fact that those acrylate-coated FBGs were unsuitable for the mandrel tests because of the rubbery feel of the coating lends some (subjective) support to this theory. As to the small changes to the response of the polyimide-coated FBG potted into the rubber pins, this might be explained by the very large number of small bubbles formed in the adhesive during that test Figure 45 . This foam will have less rigidity and consequently will transmit less of the stress of expansion to the FBG. The cause of the excessive foaming can be traced to the fact that polyurethanes cure based on residual water in the materials they contact, and the printed rubber fingers presumably had a high degree 
of residual surface hydration. (The part was created using a 3D printer that used a water bath to separate the finished part from its support structure.)
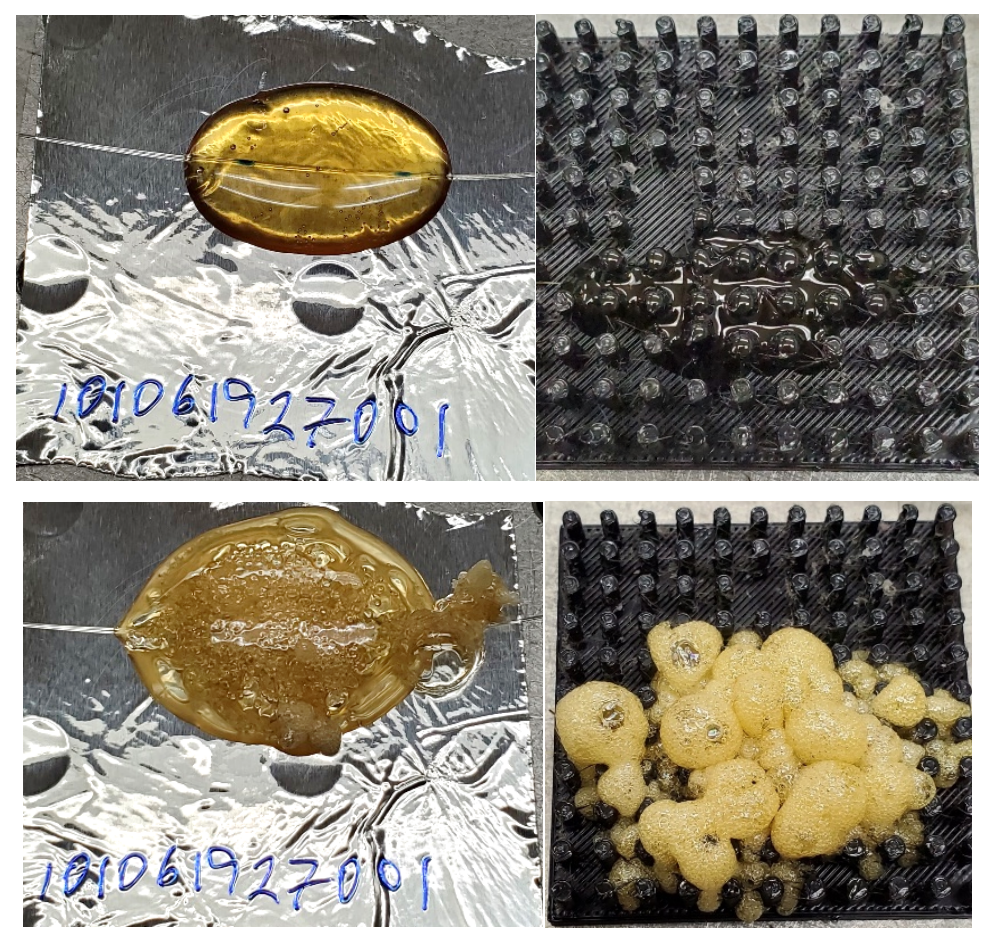

Figure 45. Images of different potted FBGs. The upper row shows the newly potted FBGs, and the bottom row shows the same FBGs after the adhesives have set. From left to right, the images correspond to the plotted results of Figures $\mathbf{4 1}$ and $\mathbf{4 2 .}$

\subsection{POTTED FBG ENVIRONMENTAL TESTS}

These speculations are supported by environmental testing of the different potted FBGs, and the results from the bare FBGs without the extra coating layer provide the simplest interpretation. These are shown in Figure 46, revealing significant shape changes with temperature, up to temperatures of $\sim 35^{\circ} \mathrm{C}$, where the behavior changes, with both FBGs show a lessening in the wavelength shift with temperature. Above the change, the polyurethane also shows an initial abrupt change with each temperature step, but then a long-term relaxation appears. The behavior of the shape parameter also changes significantly with the epoxy showing little additional shape change, whereas the polyurethane shows reductions in the shape value on each additional thermal step but with long-term drift back toward the pre-step values.

The results between the epoxy and the polyurethane also suggest that the latter has greater uptake in water from a humid environment. In fact, the epoxy shows little shape variation and almost no wavelength shift, compared to distinct changes for the polyurethane. The changes in the latter due to humidity are on a longer time scale than the $30 \mathrm{~min}$. dwell times used in the measurements, completely overcoming the stepped nature of the changes in humidity.

These results can be used to help understand the changes in the potted FBGs that also have either an acrylate or polyimide coating on them. The plots from the environmental tests on potted acrylate-coated FBGs (Figure 47) show similar morphologies, but the shape changes as the temperature was varied are clearly smaller overall. Using the earlier observations that the acrylate seems to be less tightly bound to the fiber and the rubbery consistency to the Technica samples that are potted, it is likely that this material 
serves as a buffer between the small coefficient of thermal expansion (CTE) of the silica fiber (typically $\left.\sim 5 \mathrm{ppm} /{ }^{\circ} \mathrm{C}\right)$ and that of the epoxy $\left(\mathrm{CTE} \sim 50 \mathrm{ppm} /{ }^{\circ} \mathrm{C}\right)$ and the polyurethane product that was used.
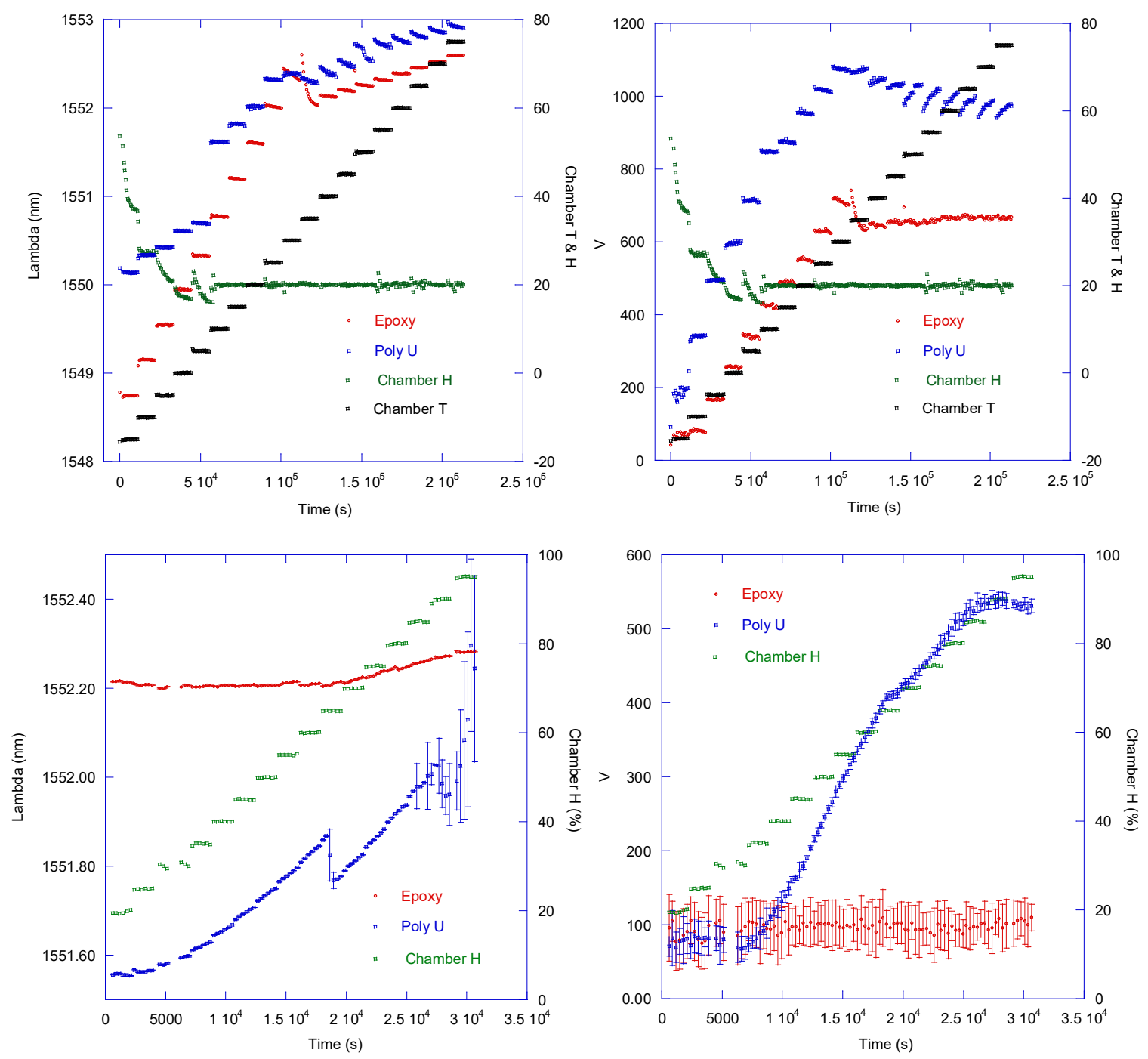

Figure 46. Environmental tests of the bare, potted FBG segments: left, wavelength response, right, shape parameter response. The upper row shows the thermal variations, and the lower shows humidity. The results are plotted as a function of time to show temporal variation during the steps. Note that the thermal steps were 3 hours long, but the humidity steps were only $\mathbf{3 0} \mathrm{min}$.

(Unfortunately, the CTE of the latter material was unavailable from the manufacturer, but in general polyurethanes have a CTE closer to that of the epoxy used, $\left.\sim 50 \mathrm{ppm} /{ }^{\circ} \mathrm{C}\right)$.

The results from the humidity tests are confusing because the results with the bare FBGs showed that the epoxy-potted device was not impacted by humidity. In addition, the earlier results with the acrylate coating also showed only small effects of humidity. Further tests are needed to understand where the water is impacting these devices.

Compared to the potted acrylate-coated FBGs, results obtained with polyurethane-potted, polyimidecoated devices were large (Figure 48). 

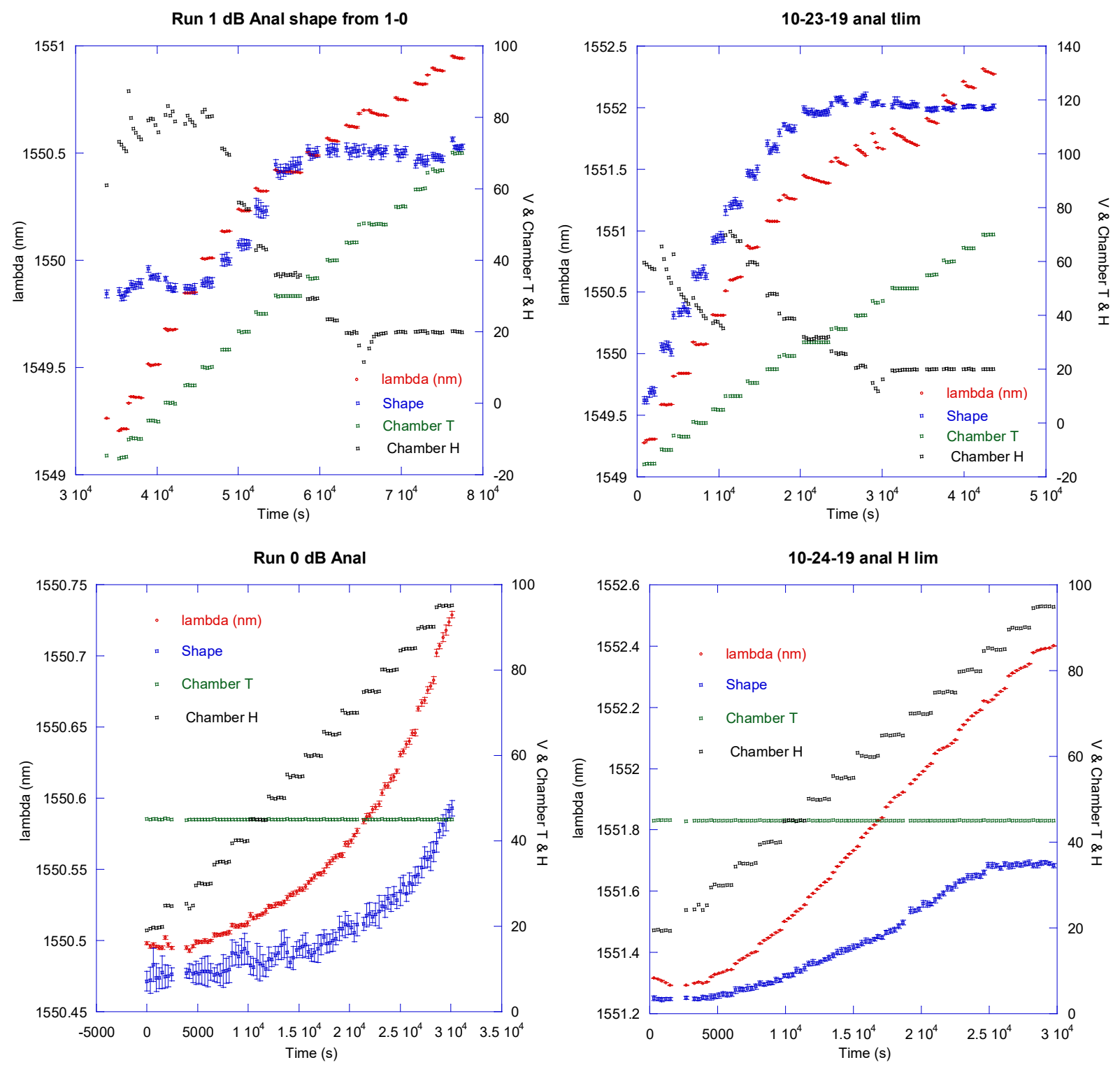

Figure 47. Thermal (upper row) and humidity (lower row) results for epoxy-potted (left) and polyurethanepotted (right) acrylate-coated Technica FBGs.

The potting results indicate that significant experimental trials will be required to understand how to create acceptable UIDs from the FBGs. This will require striking a balance between the change in the response of the individual FBGs and unacceptably large environmental changes in the finished product. Unfortunately, it is not only the CTE of the various materials but also their other mechanical properties, such as rigidity and glass transition temperature, that are important to understanding these behaviors, and unfortunately, we have already seen that the coating behaviors are vendor specific, even for coatings of the same type. 

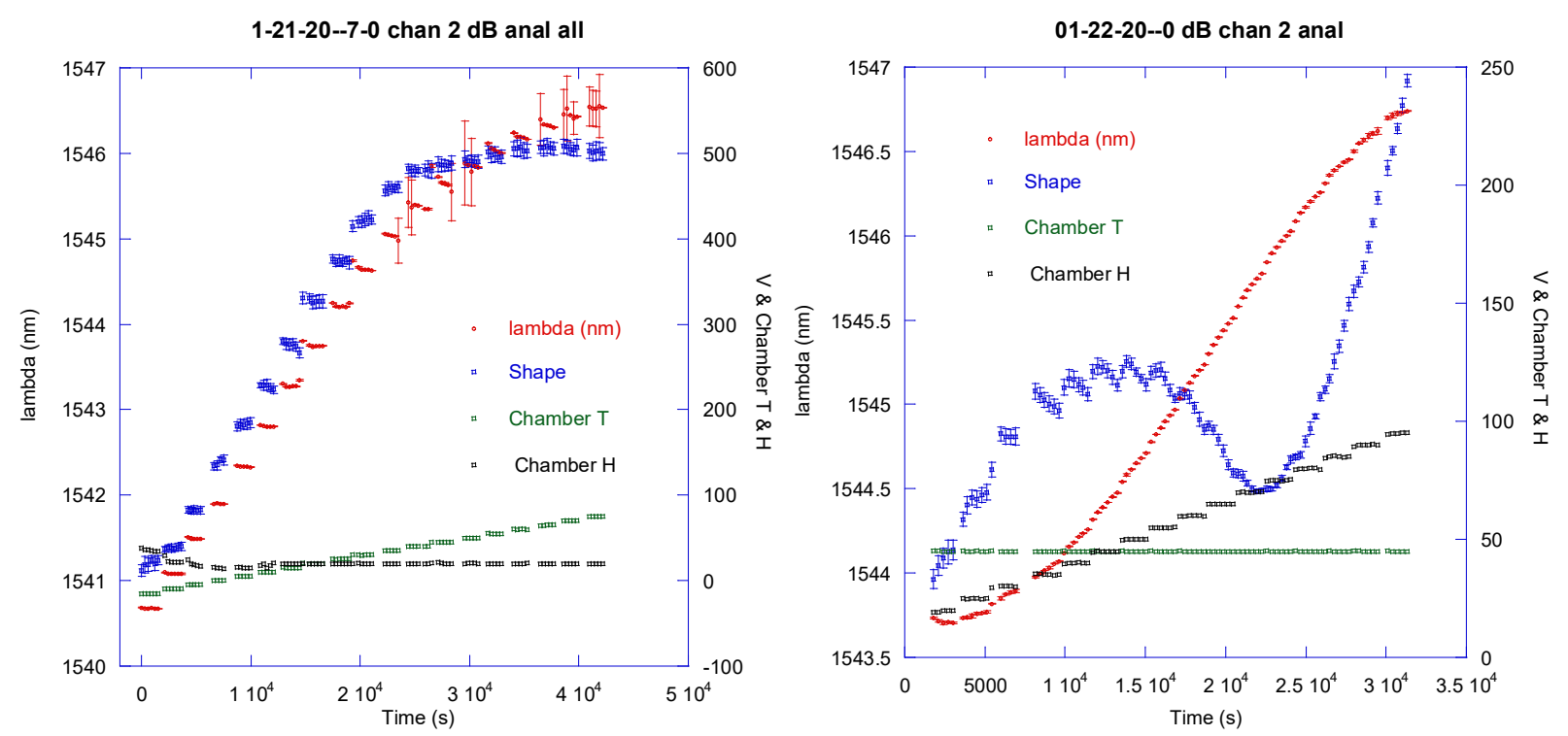

Figure 48. Temperature (left) and humidity (right) results for a polyimide-coated FBG potted in polyurethane. The changes are larger than for the acrylate-coated FBG (Figure 47), indicating tighter coupling between the potting material and the FBG.

\subsection{PRESSURE PIN TESTS}

One of the new features of this UID concept is the possibility of each stakeholder having a unique mechanical key that deterministically and repeatably alters the UIDs signature. A first test of that concept was performed by using a spring-loaded pogo pin mounted to a force meter, which was then attached to a manually activated vertical translation stage (Figure 49, left). This was used to press on the same location of one of the potted FBG gratings in three trials, each time adjusting the force in steps of $25 \mathrm{~g}$, up to a maximum of $150 \mathrm{~g}$. The results (Figure 49, right) show the shape factor from the difference-correlation metric, as a function of applied force. The data clearly cluster, with a small spread around each force value. The maximum numerical spread was 9.7, occurring at an applied force of $50 \mathrm{~g}$. Because this was meant to be a quick test, the mechanical design was suboptimal, and an improved design should improve repeatability. The design clearly allowed for some drift in the applied force, which had to be reset to zero between each run, and although each trial behaved linearly with good individual fits to a straight line, the offset and slopes varied between the different trials. Subtracting the offsets from the individual fits resulted in the tighter clustering of the points labeled "Min minus offset." These show a maximum spread at $150 \mathrm{~g}$ of $5.5 \mathrm{in}$ the shape parameter. The shape discrimination results described above indicate that the shape variation observed is comfortably within the shape variations expected from a single FBG and is smaller than those observed between different FBGs that have the same design specifications. This indicates that unique user keys should be possible. 

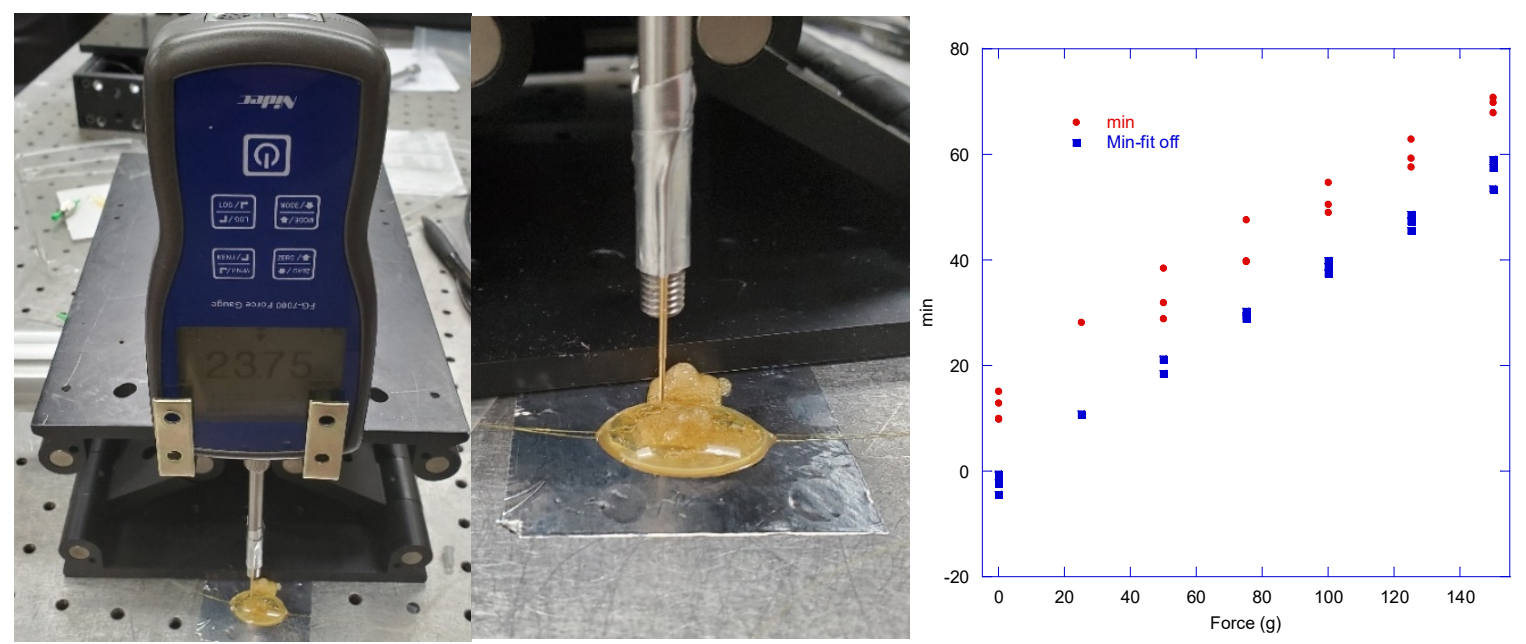

Figure 49. (Left) the overall pogo-pin test setup, and a close-up of the contact point (center). The minimum shape value vs force for the three trials (red) and after subtracting the offset of a linear fit (blue). Note that there is only a single point at $\mathbf{2 5} \mathrm{g}$ force.

\section{SIMULATIONS}

We have obtained an open-source program (FBG_SiMul V1.0) [9] designed to calculate the spectral return from stressed FBGs. The code calculates the properties of an arbitrary FBG under stress by subdividing the FBG into small regions, each of which is assumed to have uniform mechanical properties. A matrix approach is used with the response from each small region obtained from its matrix and the net result obtained by matrix multiplication of the individual results [10]. To make this work requires knowing the stress on each section of the FBG and for this, separate finite-element analysis software (ABAQUS, from Dassault Systemes) is used on a mechanical model of the FBG and its environs. To obtain sensible results requires knowing the mechanical properties (Young's modulus and Poisson's ratio) of the fiber into which the FBG is written, and these were only available as a range of values (10$70 \mathrm{GPa}$ for Young's modulus and 0.17-0.4 for Poisson's ratio) from the manufacturers of the fibers for the Technica FBGs we simulated. To refine these values, an iterative approach was used, running models with different values until the linear tension results from the simulation matched the experimental results (Figure 50), which occurred for values of $19 \mathrm{GPa}$ and 0.34 for Young's modulus and Poisson's ratio, respectively.

These values were then used for the more complex simulation of the FBG bent around a stainless-steel mandrel (using a coefficient for static friction of 0.3). Results of the shift in the center wavelength are given in Table 4 and track the experimental results well.

One of the more interesting results obtained with the code was when the fiber was bent $135^{\circ}$ around the 0.5 in. mandrel. The ABAQUS software returned the results as nonphysical due to excessive distortion in the fiber. This is consistent with the results that several FBGs broke under those conditions. While there are a number of things that could be investigated with the simulation code, we very quickly ran into computational limitations with the simple, single-post calculation requiring several hours to complete (albeit on a simple PC). That means that simulating the full complexity of the a multi-FBG system with deliberately randomized (and unknown) mechanical system details may be problematic. 


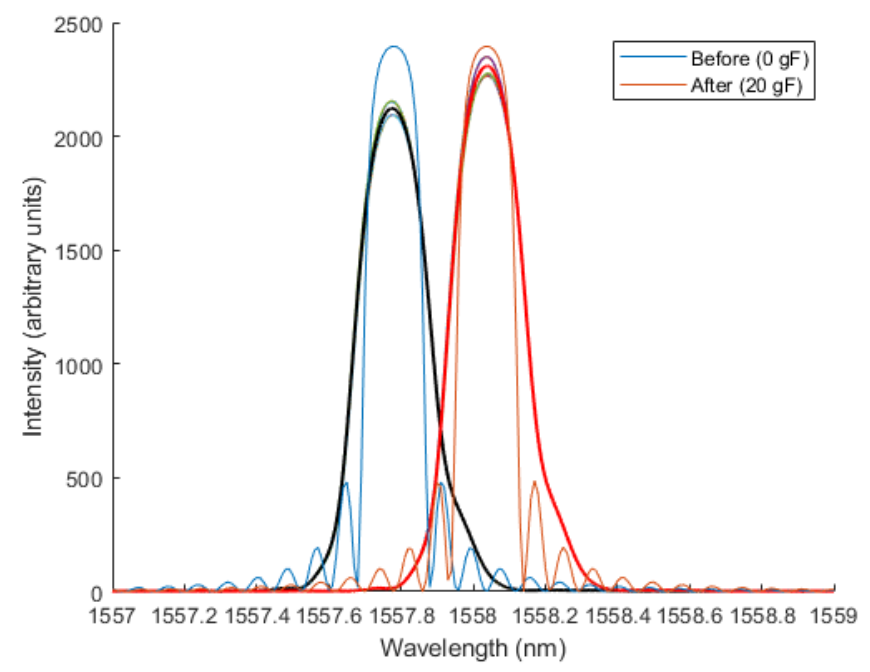

Figure 50. Simulated (thin lines) reflected signal and average measured signal (thick lines) for an FBG with a center wavelength of $1,558 \mathrm{~nm}$.

Table 4. Wavelength shifts of stressed FBGs bent around a 0.5 in. mandrel.

\begin{tabular}{|l|l|l|l|l|}
\hline $\begin{array}{l}\text { FBG wavelength } \\
(\mathbf{n m})\end{array}$ & $\begin{array}{l}\text { 9 g tension } \\
\text { wavelength shift } \\
\text { (measured) }\end{array}$ & $\begin{array}{l}\text { 9 g tension } \\
\text { wavelength shift } \\
\text { (simulated) }\end{array}$ & $\begin{array}{l}\mathbf{2 0} \text { g tension } \\
\text { wavelength shift } \\
\text { (measured) }\end{array}$ & $\begin{array}{l}\text { 20 g tension } \\
\text { wavelength shift } \\
\text { (simulated) }\end{array}$ \\
\hline 1534 & 0.1010 & 0.0935 & 0.2578 & 0.2425 \\
\hline 1550 & 0.1030 & 0.0945 & 0.2580 & 0.2451 \\
\hline 1558 & 0.0869 & 0.0925 & 0.2542 & 0.2438 \\
\hline
\end{tabular}

\section{CONCLUSIONS}

\subsection{UNIQUENESS}

To be of value, the signature returned by an individual UID must be identifiable and unique from that returned by other UIDs of the same type. At a minimum, the uniqueness must extend to a given facility, but ideally the number of possible signatures should be large enough that duplicate signatures will not be encountered across a very large installed UID base. In addition, the device must be sufficiently difficult to counterfeit to reduce the possibility of defeating the UID through its replacement with an "identical device." Without an UID manufacturing technique, the exact means that a device might be replicated surreptitiously is impossible to determine, but the results to date are promising. In Section 6.4, a number of single FBGs manufactured to the same specifications were found to provide spectral signatures that were distinct from their "identical" brethren - and for some of these, the serial numbers were different by only one, suggesting the devices were manufactured serially and presumably on the same equipment. Thus, although the sample size is small, there is some evidence that manufacturing a single FBG to match a specified spectral response is difficult, particularly for the more complex, unapodized devices.

To explore this further, consider that an individual UID will be manufactured from several $(k)$ FBGs, each with a unique signature. If we assume that the ability to distinguish different spectral returns depends on the bandwidth, $w$, of the FBG, with an amplitude discrimination, $a$, then for an interrogator with a total 
spectral range of $W$, and an amplitude discrimination capability of $A$, the number of possible unique FBG signatures $n$, is

$$
n=\frac{W A}{w a}
$$

For the UID with $k$ FBGs, the number of unique ways to populate the $n$ bins with $k$ FBGS can be determined from the binomial distribution. To do this, we ask how many unique ways are there to pull $k$ samples from a bucket containing $n$ unique items. For each pull, the bucket is replenished (e.g., there are $n$ possible choices for each pull). This value is given by the binomial coefficient:

$$
\left(\begin{array}{l}
n \\
k
\end{array}\right)=\frac{n !}{k !(n-k) !}
$$

Taking a conservative individual FBG bandwidth of $w=0.25 \mathrm{~nm}$ (current FBGs are well overresolved with $\sim 250$ bins) for an interrogator bandwidth of $W=40 \mathrm{~nm}$ for 160 spectral values, and assume an ability to resolve $3 \mathrm{~dB}$ differences out of 40 for 13 amplitude values, then $n=2,080$. If we use five FBGs then there are

$$
\left(\begin{array}{c}
2080 \\
5
\end{array}\right)=\frac{2080 !}{5 ! 2075 !}=3.2 \times 10^{14}
$$

possible combinations! This is sufficiently large to make accidental creation of similar UIDs unlikely, and combined with the variations in samples of "identical" FBGs indicates, it will be very difficult to replicate a given UID, even without further altering the FBG signatures.

\subsection{SPECTRAL SPACING}

The preceding discussion treats the signatures of the UID component FBGs as uniquely separable and means that the additional complexity of overlapping the signatures is not required. One of the immediate implications of this finding is that the difference-correlation technique remains a viable analysis tool, and the individual shapes associated with each of the constituent FBGs can be used as the overall UID signature. The benefits compared with using a single long shape should be immediate. First it allows for individual shifts to the peak locations of the constituent FBGs (which may have different stressor fields as environmental conditions change), and second, it provides a simple multifactor metric that includes the complexity of the individual FBGs without having to disentangle subtle changes engendered by overlapping signatures.

\subsection{FIELD INTERROGATOR}

The cost of the interrogator used in this work is an order of magnitude higher than the few thousanddollar target price for a field instrument, and not having an affordable readout device would clearly make FBG-based UIDs impracticable. The higher performance of the FAZT-I4G was selected to help determine the minimum requirements of a fieldable unit. In fact, most of the work was performed using only a quarter of the resolution available in the raw spectra, and the evaluation of gratings with different parameters indicate that the overall shapes can be tailored to have significantly broader features than the typical $0.25 \mathrm{~nm}$ width used for most of the measurements presented above. It is thus reasonable to assume that UIDs can be designed for use with interrogators with at least an order of magnitude reduction in spectral resolution.

With that information, an initial target cost is about $\$ 5 \mathrm{~K}$, and the requirements that a fieldable unit should exhibit approximately the same characteristics as the laboratory interrogator: tunability across the FBG 
operating range, narrow spectral linewidth, and spectral stability. We looked at products from two manufacturers of tunable laser diode sources, PurePhotonics and Freedom Photons. Each of these companies offer fiber-coupled, compact, narrow-linewidth, tunable, solid-state laser diode packages with costs commensurate with a total interrogator price around the initial target. We were able to evaluate the performance of a PurePhotonics PPCL550 [11] with respect to the wavelength tunability and linewidth (Figure 51). The results are encouraging with linear tuning over the 1,530-1,565 nm and a sub-20 pm linewidth.

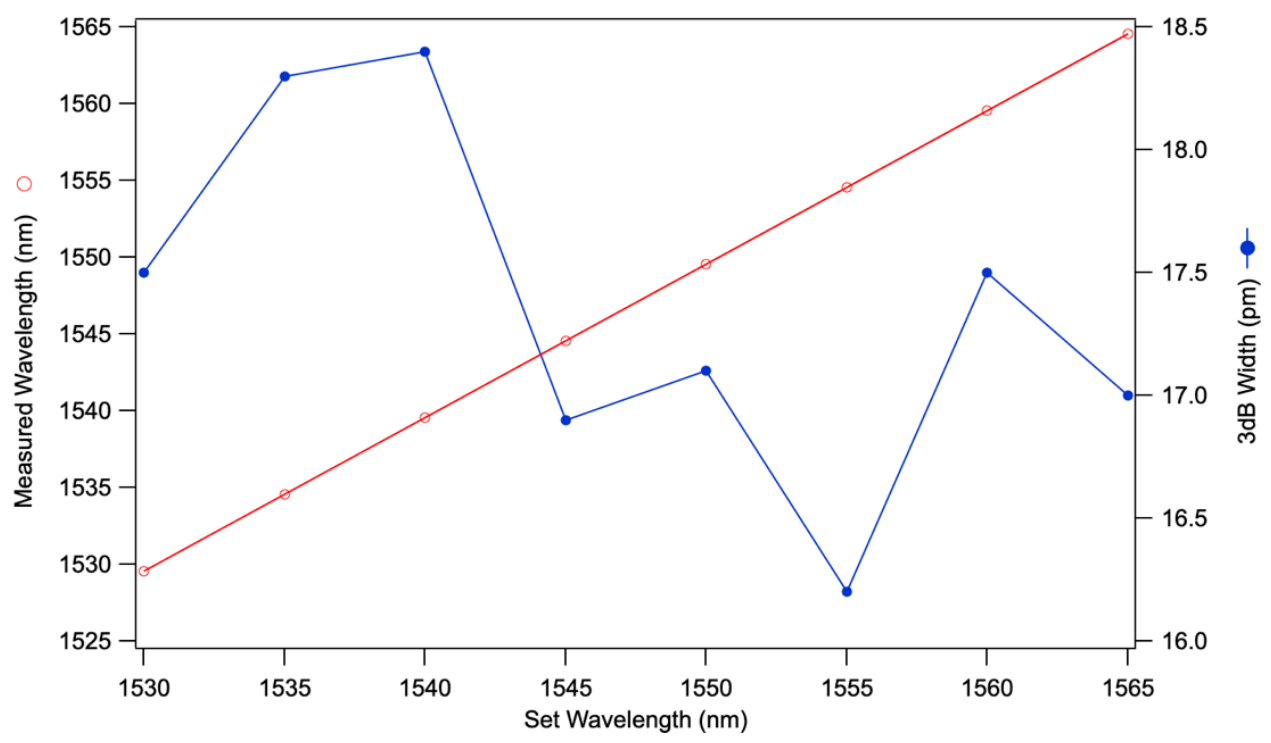

Figure 51. Wavelength and linewidth measurements for the PurePhotonics PPCL550 compact tunable laser diode.

The PPCL550 cost is approximately $\$ 3,000$ /unit including various calibrations and an enclosure. For a possible future packaged FBG interrogator, purchasers may opt out of the enclosure option and leverage bulk purchasing to push the unit cost to approximately $\$ 2,000 /$ unit.

In comparison, the Freedom Photonics FP3015C [12] is a butterfly-style laser diode package currently in the final stages of product development. We could not obtain a test sample for characterization, yet from manufacturer-supplied data sheets, the performance is similar (if not better) than the PurePhotonics PPCL550 with the advantage of a smaller form factor. The projected unit cost is $\$ 3,500 /$ unit with lead times approaching 6 months.

Lower resolution and cost were already demonstrated as successful for a tamper indicating device designed to enclose integrated circuits [13]. That readout device was based on using a broadband light source coupled with a portable spectrometer. Whatever the final approach, it is clear that a suitable device can be realized, and further research into this is only appropriate as the performance of an UID is better understood.

\subsection{ENVIRONMENTAL ISSUES}

The environmental results of the unpotted FBGs indicate that acrylate is a better choice than the polyimide because the latter's obvious absorption of water as the humidity increases. Clearly, the less the system changes with the environment, the better. 
For the potted FBGs, the results also point toward the acrylate coating, which changed less in the shape than the ones coated with polyimide as the environmental conditions were varied. In fact, the Technica polyimide-coated FBGs potted in expanding polyurethane showed exceptionally large changes in the shape and wavelength with temperature, as did bare FBGs potted in either the epoxy or polyurethane. Further, the results also showed almost continuous change in wavelength with time as the humidity was changed, indicating long-term (greater than the $30 \mathrm{~min}$. soak times) impacts of small changes in relative humidity. It is not clear if this was due to absorption of water by the potting compound or migration of the water to the FBG from the exposed fiber extending beyond the potted region of the system.

Overall, these findings indicate that considerable effort will need to go into selecting and testing different potting materials. One option may be to use low-CTE ceramic potting materials with uncoated FBGs. These materials match the CTE of silica quite well and could significantly lessen the impact of environmental changes. Alternately, low-CTE epoxies (approximately only twice that of silica) combined with the ameliorating effect of the acrylate coating may work as well and provide a more robust device.

Overall, the expanding polyurethane appears to be a poor choice, and this raises the issue of whether a potting compound can be found that also provides a randomized stressor field on setting. Fortunately, the findings on the uniqueness of the individual FBGs may indicate that adding such a field during the manufacturing process is likely unnecessary. The individuality of each of the large number of FBG samples tested indicates that finding a replacement for an individual FBG will be difficult. For a baseline UID design with five FBGs, finding five replacements should prove a daunting challenge. That said, the concept of altering the response of the FBGS in an UID has not been abandoned. The mandrel-wrap results that show shape changes under tension when a portion of an FBG contacts a mandrel indicate that altering the spectral return should be as simple as sequentially altering tension on a grating as it is potted and threaded through a pin array.

\section{SUMMARY}

In conclusion, the results reported here provide guidance for a successful path forward. They indicate that a five FBG UID should provide a device that will be extremely difficult to replicate. This uniqueness is achieved even if the spectra from the constituent FBGs do not overlap, allowing the use of simple difference-correlation of the individual FBGs as a validation tool. That validation can be achieved after interrogating the UID with modestly priced, fieldable hardware. Finally, the concept of using individual stakeholder keys is valid and will provide further deterrence to defeating the device.

The findings do indicate that the future path includes some changes from the initial concept. Primary among these is that achieving a randomized stressor field through the use of a potting compound that changes its dimensions on setting is unlikely. The limited number of candidate materials, compounded by requirements on environmental stability, indicate that a direct mechanical approach is better suited to achieving this goal. Further, the sample-dependent signatures of individual FBGs coupled with vendorspecific performance of FBG recoating materials and the subtleties of their interactions on potting under stressed conditions suggests a largely empirical approach to the UID design.

\section{REFERENCES}

1. M. Ramakrishnan, et al., "Overview of Fiber Optic Sensor Technologies for Strain/Temperature Sensing Applications in Composite Materials," MDPI, January 15, 2016. [Online]. Available: https://www.mdpi.com/1424-8220/16/1/99/htm.

2. Faz Technology Inc., Model I4G Interrogator. [Online]. Available: www.faztechnology.com/products/interrogators/fazt-i4g/. 
3. Corning, ClearCurve ZBL Optical Fiber. [Online]. Available:

www.corning.com/media/worldwide/coc/documents/Fiber/PI1464 07-14 English.pdf.

4. R. T. Schermer, J. H. Cole, "Improved Bend Loss Formula Verified for Optical Fiber by Simulation and Experiment," IEEE J. of Quant. Elec., vol. 43, 899-909, 2007.

5. Associated Environmental Systems, LH/LHE Series. [Online]. Available: https://www.associatedenvironmentalsystems.com/series/LH.

6. Phidgets Inc., Model 1125 Humidity/Temperature Sensor. [Online]. Available: www.phidgets.com/?tier=3\&catid=14\&pcid=12\&prodid=96.

7. Nidec-Shimpo Corp., Model FG-7000. [Online]. Available: www.instruments.nidecshimpo.com/en/fg-7000-digital-gauge/.

8. P. Kronenberg, et al., "Relative humidity sensor with optical fiber Bragg gratings," Opt. Lett., vol. 27, pp. 1385-1387, 2002.

9. G.F. Pereira, M. McGugan, L. P. Mikkelsen, [Online]. Available: github.com/GilmarPereira/FBG_SiMul.

10. G.F. Pereira, M. McGugan, L. P. Mikkelsen, "FBG_SiMul V1.0: Fiber Bragg grating signal simulation tool for finite element method models," SoftwareX, vol. 5, pp. 163-170, 2016, http://dx.doi.org/10.1016/j.softx.2016.08.001.

11. Pure Photonics, Model PPCL550. [Online]. Available: www.pure-photonics.com/product-solutions.

12. Freedom Photonics, Model FP3015C. [Online]. Available: freedomphotonics.com/freedomphotonics-products/lasers-and-laser-sources/1550nm-tunable-laser-fp3015c/.

13. M. Thiel, G. Flachenecker, W. Schade, G. Gorecki, A. Thoma, R. Rathje, "Planar ultra thin glass seals with optical fiber interface for monitoring tamper attacks on security eminent components," Optics and Lasers in Engineering, vol. 98, pp. 89-98, 2017. Available: http://dx.doi.org/10.1016/j.optlaseng.2017.06.007. 